\title{
A WEARABLE 1.6GHZ NON-INVASIVE MIDFIELD WAVE-BASED BLOOD GLUCOSE SENSOR
}

\author{
A Thesis \\ presented to \\ the Faculty of California Polytechnic State University, \\ San Luis Obispo
}

\author{
In Partial Fulfillment \\ of the Requirements for the Degree \\ Master of Science in Electrical Engineering
}

by

Danielle Nishida

March 2015 
(C) 2015

Danielle Nishida

ALL RIGHTS RESERVED 
COMMITTEE MEMBERSHIP

TITLE:

AUTHOR:

DATE SUBMITTED:

COMMITTEE CHAIR:

COMMITTEE MEMBER:

COMMITTEE MEMBER:
A Wearable 1.6GHz Non-Invasive Midfield Wave-

Based Blood Glucose Sensor

Danielle Nishida

March 2015

Dr. Tina Smilkstein, Ph.D.

Assistant Professor of Electrical Engineering

Dr. Jane Zhang, Ph.D.

Professor of Electrical Engineering

Dr. Lily Laiho, Ph.D.

Professor of Biomedical Engineering 


\begin{abstract}
A Wearable 1.6GHz Non-Invasive Midfield Wave-Based Blood Glucose Sensor Danielle Nishida
\end{abstract}

A non-invasive glucose sensor has been sought after by millions of Americans living with diabetes and scientists alike. Current blood glucose sensors approved by the FDA involve an invasive collection technique coupled with costly chemical analysis materials, which continue to bring pain to the growing diabetic population in America. Previous research of non-invasive sensing methods has shown promise, but the difficulty of transmitting power through tissue has degraded the sensing consistency and accuracy needed to reach the consumer market. This has been overcome with recent research in midfield wave power transmission at Stanford University, which has provided an opportunity for investigating a new, non-invasive glucose sensing technique. The design proposed in this thesis uses a slot array antenna transmitting through the human arm in the UHF (Ultra High Frequency) range at a wavelength absorbed by glucose to investigate the relationship between blood glucose levels and received power from a loop antenna receiver. The complete system incorporates an RF mixer, a Wilkinson power divider, and an RF detector to enable use with Cal Poly's lab equipment. Changes in blood glucose levels are tracked within an $\mathrm{R}$ value of 0.91 when the testing setup maintains consistency in physical placement of the transmitter and receiver. Ten measurements over the course of seven hours accurately sensed changes in the subject's blood glucose levels with two outlier data points. The system provides a vehicle for further investigation into a new, non-invasive blood glucose sensing method to improve the quality of life for millions around the world. 


\section{ACKNOWLEDGEMENTS}

I would like to thank my parents, sister, and boyfriend for providing endless support and encouragement during my pursuit of the 4+1 Honors Program in Electrical Engineering. The support and guidance from Dr. Tina Smilkstein was also invaluable during this learning experience, and I thank her for the ideas, feedback, and discussions throughout the entire process.

I also extend my thanks to additional colleagues and professors who provided

help during the designing and testing of my thesis; namely Dr. Arakaki, Dawei Zhang, and Michael Harriman. 


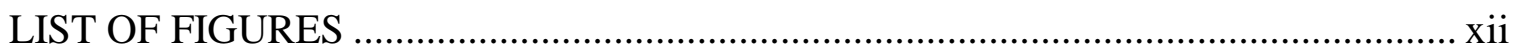

Chapter 1: Introduction to Diabetes and Glucose Sensing .............................................

1.1 Glucose Monitoring Methods ………….....................................................

1.1.1 Invasive or Minimally Invasive Glucose Monitoring ....................................4

1.1.2 Non-Invasive Glucose Monitoring ………………...................................6

1.1.2.1 Reverse Iontophoresis ...................................................................

1.1.2.2 Absorption Spectroscopy ………………........................................

1.1.2.3 Photoacoustic Spectroscopy ..............................................................

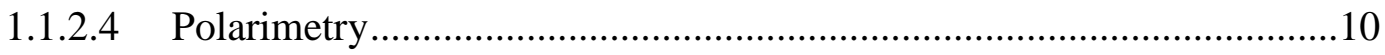

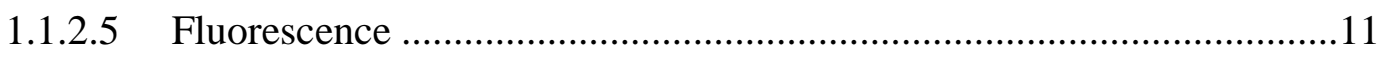

1.1.2.6 Raman Spectroscopy ......................................................................11

1.1.2.7 Metabolic Heat Conformation ..........................................................12

1.1.2.8 Thermal Emission Spectroscopy..........................................................13

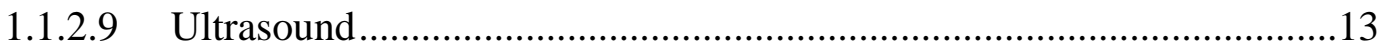

1.1.2.10 Bioimpedance Spectroscopy ……………..........................................14

1.1.2.11 Electromagnetic Sensing.................................................................15

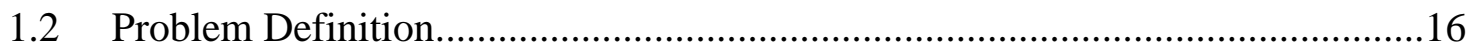




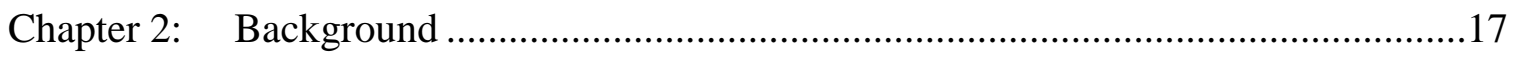

2.1 Potential New Methods for Electromagnetic Sensing Technologies...................17

2.1.1 Determining Optimal Frequency ………………........................................17

2.1.1.1 Power Transfer Efficiency ................................................................18

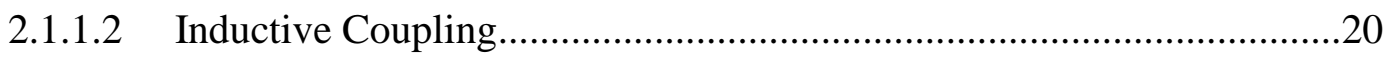

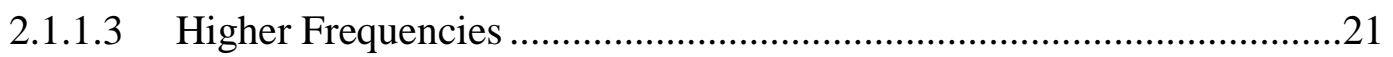

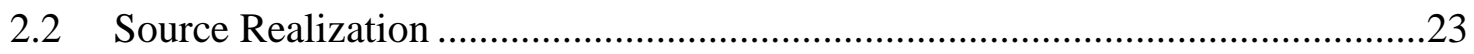

2.3 Overall Results from Ho et al. [27] and Kim et al. [30] .....................................24

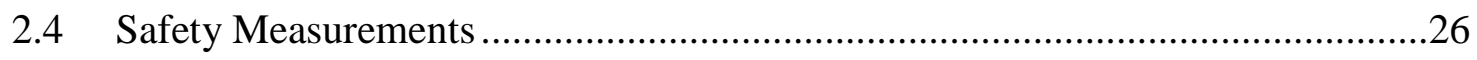

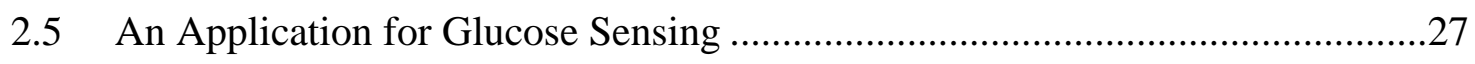

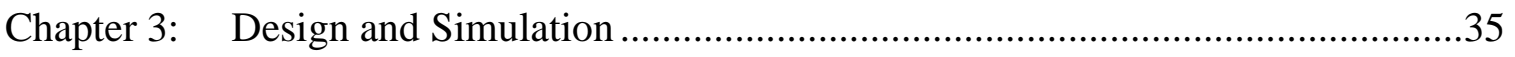

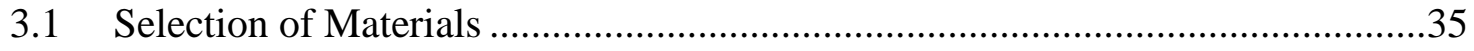

3.2 Preliminary Source Excitation Designs ..............................................................

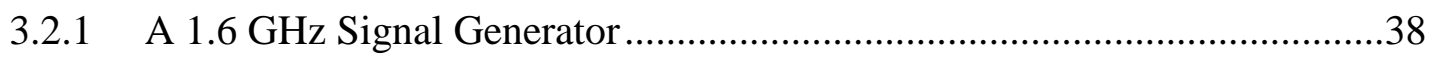

3.2.2 Four-Output Wilkinson Power Divider ………………................................

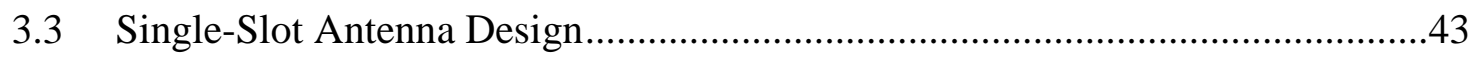

3.3.1 Increasing Radiation Efficiency ................................................................43

3.3.2 Impedance Matching for Single-Slot Antenna Design .................................46

3.4 Slot Array Antenna Characterization............................................................52

3.4.1 Determining the Directionality of the Slot Array Antenna............................55 
3.4.2 Three-Dimensional Analysis of Slot Array Antenna Propagating through

Biological Tissue 61

3.5 Receiver Design for Slot Array Antenna Transmitter .66

Chapter 4: Glucose Sensor Test Setup and Results .69

4.1 System Assembly .69

4.2 Testing the Signal Transmitter. .69

4.2.1 RF Mixer and Bandpass Filter Test Setup and Results .70

4.2.2 Wilkinson Power Divider Test Setup and Results .72

4.2.3 Slot Array Antenna Test Setup and Results........................................... 76

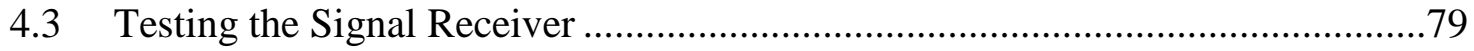

4.3.1 Loop Antenna Test Setup and Results ................................................. 79

4.3.2 RF Detector Test Setup and Results ....................................................81

4.4 System Test Setup and Results ................................................................ 83

4.4.1 System Test on Blood Mimicking Phantoms ........................................87

4.4.2 System Test on Human Subjects.............................................................91

Chapter 5: Conclusion and Future Considerations ................................................101

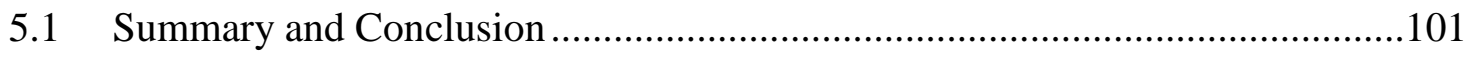

5.2 Future Considerations .......................................................................... 103

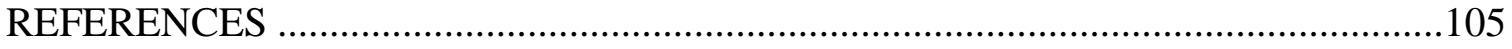

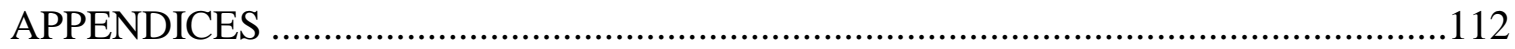


I. Fabricating Blood Mimicking Phantoms.........................................................112

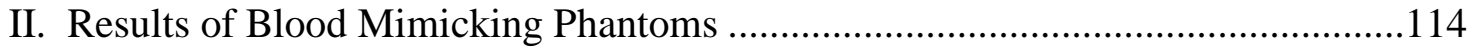




\section{LIST OF TABLES}

Table

Page

Table 1: Three-pole Debye-Drude model determined for glucose in blood plasma......... 28

Table 2: Relation between blood glucose concentration, blood relative permittivity, and the maximum radiated power as measured by ADS.

Table 3: Substrate comparisons between FR-4/Duroid 5880 and RO4350 from

Rogers Corporation.

Table 4: Simulated S-parameters for the outputs of the 4-way Wilkinson power divider show near ideal values. Due to reciprocity, the output ports have the same power when looking from the source to the output.

Table 5: Various sizes for shapes $S 1, S 2$, and $S 3$ were tested to find the maximum power transfer efficiency in free space.

Table 6: Based on the input power and radiated power as calculated by ADS, the radiation efficiency was determined to choose the optimal slot antenna design. 45

Table 7: Simulated S-parameters for the slot array antenna in free space.

Table 8: Input port setup determined for optimal directionality per simulation results.

Table 9: Setup of layers simulated with their thicknesses and electrical parameters.

The three types of blood were simulated one by one in combination with all other

layers. .

Table 10: EMPro simulation results demonstrated the inverse relationship between glucose and received current density, magnetic field, and electric field. 
Table 11: Measured parameters of the 4-way Wilkinson Power divider. Each measured parameter from the input to output were near $-6 \mathrm{~dB}$, as desired by the design

Table 12: Receiver placement around the slot array antenna used to determine the optimal location with maximum received signal strength intensity for future

measurements.

Table 13: Measured blood glucose and RSSI were recorded for the first human subject with two orientations of the receiving antenna.

Table 14: Characteristics of each test subject led to the estimation of their muscleto-fat ratio for a cross-section of their upper arm. 96

Table 15: Each test subject underwent two or three tests to determine a trend in measurements.

Table 16: List of materials necessary for creating blood mimicking phantom. 112

Table 17: Measurements of dextrose necessary for achieving a variety of blood glucose levels

Table 18: Various blood phantoms were tested to fluctuate the received signal strength based on changing electrical properties.

Table 19: The expected behavior of RSSI based on different blood glucose levels was not demonstrated based on the blood phantom measurements. 


\section{LIST OF FIGURES}

Figure

Figure 1: The key factors differentiating Type 1 and Type 2 diabetes involve the

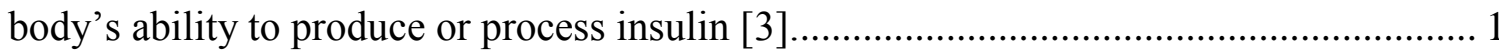

Figure 2: Summary of invasive, minimally invasive, and non-invasive blood

glucose monitoring technologies.

Figure 3: Photoacoustic sensing methods implement an ultrasound transducer to detect ultrasonic waves created by pulsing light on the body.

Figure 4: Block diagram of a 2-port model displaying relations between the source and receiver that are necessary for understanding inductively coupled coils. 18

Figure 5: Electric current density realizations (left: source, right: receiver) for necessary field propagations through multiple layers of biological materials. 24

Figure 6: Slot array layout (left) and microstrip array (right) realized by Kim et al. [30] for maximum power transfer through greater than $5 \mathrm{~cm}$ of biological tissue. The microstrip layer marks the four input ports by the overlapping blue dots. Figure 7: Image of slot array antenna with microstrip layer (yellow) superimposed on slot layer (red) designed by Kim et al [30].

Figure 8: The antenna can produce different angles of output direction based on the phases of the four inputs. 26

Figure 9: The 3-D simulation model of two microstrip patch antennas separated by a $1 \mathrm{~cm}$ air gap and $5 \mathrm{~cm}$ thick block of glucose was modeled to determine the optimal operating frequency for a glucose sensor. 
Figure 10: Based on EMPro simulations through $5 \mathrm{~cm}$ of glucose, the greatest absorption of energy occurred at $1.6 \mathrm{GHz}$. 30

Figure 11: As previously stated, increased blood glucose concentration inversely affects the dielectric permittivity of blood and therefore decreased radiated power received.

Figure 12: Current density of simulated blood glucose concentrations of 134, 95, and $72 \mathrm{mg} / \mathrm{dL}$ respectively 32

Figure 13: Cross-section of the upper arm and targeted position of the slot array antenna source and receiver with angled wave propagation intensity. 33 Figure 14: Bandpass filter designed for extracting unwanted output signals from the frequency mixer [37].

Figure 15: The 2-way Wilkinson power divider schematic designed in ADS. The microstrip lengths and widths were calculated to match the desired impedance at $1.6 \mathrm{GHz}$. 40

Figure 16: The S-parameters resulting from the simulation of a 2-way Wilkinson power divider proves the anticipated $3 \mathrm{~dB}$ loss at the two outputs at $1.6 \mathrm{GHz}$. 41 Figure 17: ADS schematic of the 4-way Wilkinson power divider derived from the 2-way splitter. 42 Figure 18: Corresponding layout of 4-way Wilkinson power divider with the single input port (Port 1) at the left and four separate outputs (Ports 2-5) on the right. The vertical microstrips are $100 \Omega$ resistors to dissipate reflections at the outputs 
Figure 19: The single slot antenna design was configured in six different ways based on size parameters of shapes $\mathrm{S} 1, \mathrm{~S} 2$, and $\mathrm{S} 3$. The yellow semi-circle represents the top microstrip layer, and the red arc and line represent the bottom slot

layer 44

Figure 20: A graphical representation of the radiated power based on input power for all six designs shows the variation in each of the two parameters. 46

Figure 21: To find the optimal point of excitation for the single slot antenna design, $\mathrm{S}_{11}$ parameters were acquired at the identified nine points around the microstrip curve. 48

Figure 22: The simulated $S_{11}$ parameters of all four layout designs (L1, L4, L5, and L6) determined the best input placement for minimal signal reflection. Figure 23: Physical layout of design L4 (left) and its current density (right). The maximum current density achieved was $11.91 \mathrm{~A} / \mathrm{m}$. 50 Figure 24: The antenna pattern of the single slot design shows a symmetrical pattern about the z-axis. Far field measurements were recorded for the cut plane at a polar angle of 60 degrees indicated by the green line. 51 Figure 25: At a far field plane of 60 degrees, the maximum gain of the antenna was near $20 \mathrm{dBi}$. The radiation intensity of the single slot antenna was 0.008 $\mathrm{W} / \mathrm{sterad}$, and the maximum magnetic far field was $-7.78 \mathrm{E}-5 \mathrm{~A} / \mathrm{m}$. 51 Figure 26: ADS layout design and 3-D representation of the full slot array antenna. The yellow shapes indicate the microstrip layer, and the dark red indicate the ground plane with slot shapes removed 52 
Figure 27: ADS simulation of the slot array achieved a current density of 17.77

$\mathrm{A} / \mathrm{m}$. 53

Figure 28: The antenna pattern of the full slot array in free space shows what appears to be a symmetrical radiation pattern above and below the plane of the antenna. 54

Figure 29: Gain, radiation, and magnetic far field patterns from a far field cut at a zero degree polar angle. 55

Figure 30: Simulation setup of full slot array antenna and $4 \mathrm{~cm}$ layer of blood (red) separated by $1 \mathrm{~cm}$ of air (unshaded). 56 Figure 31: Changing magnetic field magnitudes under the $4 \mathrm{~cm}$ layer of blood after incrementing the phase of input port 1 by 90 degrees ( 0 degrees, 90 degrees, 180 degrees, 270 degrees, respectively). 58

Figure 32: Varying the input phases of the remaining three ports (ports 2, 3, and 4, respectively) by 90 degrees shows the effect of the input phase on the magnetic fields at distances near $5 \mathrm{~cm}$ away.

Figure 33: The magnetic field at the interface between air and blood displays the direction of the strongest current density (left) and the resulting current density at the bottom surface of the blood layer (right).

Figure 34: Snapshots of the magnetic field propagating through the blood layer at the intersection of largest current density.

Figure 35: Physical setup for simulation environment. The antenna was separated by $1 \mathrm{~cm}$ of air, followed by $3 \mathrm{~mm}$ of skin, $2 \mathrm{~mm}$ of blood, $2 \mathrm{~mm}$ of fat, $3 \mathrm{~cm}$ of muscle, $2 \mathrm{~mm}$ of fat, $2 \mathrm{~mm}$ of blood, and $3 \mathrm{~mm}$ of skin. 63 
Figure 36: Magnitude of the current density on the receiving side of the simulated biceps based on the optimal input phase settings. The simulations are in order from highest to lowest blood sugar, and the resulting magnitudes are 0.03912, 0.04202, and $0.04368 \mathrm{~A} / \mathrm{m}^{2}$, respectively. 64

Figure 37: Magnitude of the magnetic field on the receiving side of the simulated biceps based on the optimal input phase settings. The simulations range from the (a) highest blood sugar level with $0.03546 \mathrm{~A} / \mathrm{m}$, to (b) medium blood sugar level with $0.04133 \mathrm{~A} / \mathrm{m}$, and (c) lowest blood sugar level with $0.04785 \mathrm{~A} / \mathrm{m}$. 64 Figure 38: Magnitude of the electric field on the receiving side of the simulated biceps based on the optimal input phase settings. The simulations range from the (a) highest blood sugar level with $2.104 \mathrm{~V} / \mathrm{m}$, to (b) medium blood sugar level with $2.105 \mathrm{~V} / \mathrm{m}$, and (c) lowest blood sugar level with $2.114 \mathrm{~V} / \mathrm{m}$. 65

Figure 39: Based on the simulation for the medium level of blood glucose, the magnitude of the magnetic field at the surface of the skin furthest from the antenna determined a generic location for the output.

Figure 40: Animation stills of the propagating magnetic field through all the described biological layers. The animation images are captured in increments of 30 degrees and range from 0 to 150 degrees. 66 Figure 41: Block diagram of the entire sensing system, beginning with the RF mixer and ending with the Arduino Uno Processor

Figure 42: RF mixer implementing a local oscillator and RF input signal used to create a $1.6 \mathrm{GHz}$ output for the slot array antenna. 
Figure 43: Bandpass filter centered at $1.6 \mathrm{GHz}$ used to remove harmonics from the output of the RF mixer.

Figure 44: Due to the nature of the RF mixer, multiple signals were generated across $1.3 \mathrm{GHz}-1.9 \mathrm{GHz}$, but filtered well with the designed bandpass filter. 72 Figure 45: 4-output Wilkinson power divider designed for splitting a single input into four different outputs with equal loss.

Figure 46: Measured signals at each of the four output ports of the 4-way Wilkinson divider with $10 \mathrm{dBm}$ RF mixer input signals. Ports 2 through 5 correspond to (a) through $(\mathrm{d})$, respectively. 74 Figure 47: Possible sources of signal loss at each output included potential fringing at sharp corners and coupling between nearby microstrips. 75 Figure 48: Measured signals at each of the four output ports of the 4-way Wilkinson divider with $15 \mathrm{dBm}$ RF mixer input signals. Ports 2 through 5 correspond to (a) through (d), respectively 76

Figure 49: Slot array antenna with connecting coaxial cables soldered to the microstrip layer (left) and ground/slot layer (right). 77

Figure 50: Loop antenna created for receiving the $1.6 \mathrm{GHz}$ transmitted signal. 80 Figure 51: The loop antenna's response at 1.6 GHz showed the desired resonance with $-23.5 \mathrm{~dB}$ of loss. 81

Figure 52: The RF detector demo board with LT5534 detector chip by Linear Technologies was implemented for its broad detection range. 82 Figure 53: The RF detector's received power and DC voltage output linearly correlated by $41 \mathrm{mV} / \mathrm{dB}$ [37]. 83 
Figure 54: RSSI measurements were taken in free space with the receiver coil centered above input 1 on the slot array transmitter. 84 Figure 55: The loop antenna receiver was positioned at varying distances from the transmitter to characterize the system in free space 85 Figure 56: Received signal strength decreased as the receiver moved further away from the antenna. 85

Figure 57: Full system diagram with test sample placement specified between the transmitter and receiver. 86

Figure 58: A wooden frame was constructed out of balsa wood to maintain the necessary $1 \mathrm{~cm}$ air gap during testing. 86

Figure 59: Top view and side view of the two fabricated blood phantoms. One phantom contained no added glucose (left) while the other contained 5 grams of added glucose (right) 89 Figure 60: The test setup for the initial blood glucose phantoms placed the entire blood phantom on top of the transmitting antenna with the receiver placed directly on the phantom 90 Figure 61: The test setup on human subjects involved tying the transmitter and receiver to the biceps with an elastic band. 92 Figure 62: Measurements from the receiving antenna in the first orientation showed the expected inverse relationship between glucose and RSSI. 94 Figure 63: Measurements from the receiving antenna in the second orientation showed the expected inverse relationship between glucose and RSSI with two outliers 94 
Figure 64: The measured RSSI and blood glucose levels varied across each test subject, yet 5 of the subjects' data reveals the expected inverse relationship

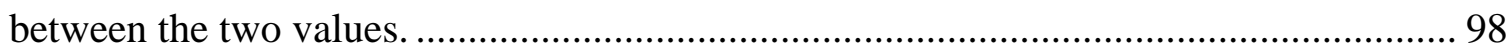

Figure 65: With the exception of a few data points, the sensed and measured blood glucose levels for Subjects A, B, and C display a trend similar to the expected relation. 99 Figure 66: Subjects with smaller arm circumferences were found to have varying measurements attributed to distance between the receiver and arm. 100 


\section{Chapter 1: Introduction to Diabetes and Glucose Sensing}

The American Diabetes Association reports that 29.1 million Americans, or about one out of 11 people, currently have diabetes [1]. Even more alarming, 89 million Americans are prediabetic, and nine out of ten of them are unaware of this fact. Current dietary trends and decreased physical activity in today's world of convenience foster such high populations for this chronic disease. Unfortunately, these conditions will cause 15$30 \%$ of prediabetics to develop Type 2 diabetes within 5 years, and their risk of death will resultantly increase to $50 \%$ greater than adults without diabetes [1].

When individuals develop diabetes, their body can no longer produce a hormone called insulin (Type 1 diabetes) or their body can produce insulin but has difficulty using it effectively (Type 2 diabetes), as illustrated in Figure 1 [2]. The importance of insulin involves the chemical reaction it causes in the body - insulin allows glucose from ingested food to pass from the blood stream into the body's cells, thus producing energy. Diabetes causes an abundance of glucose in the blood since it is not transported to the body's cells, and this profusion of glucose can cause blindness, kidney failure, heart disease, stroke, or loss of toes, feet, or legs [2].

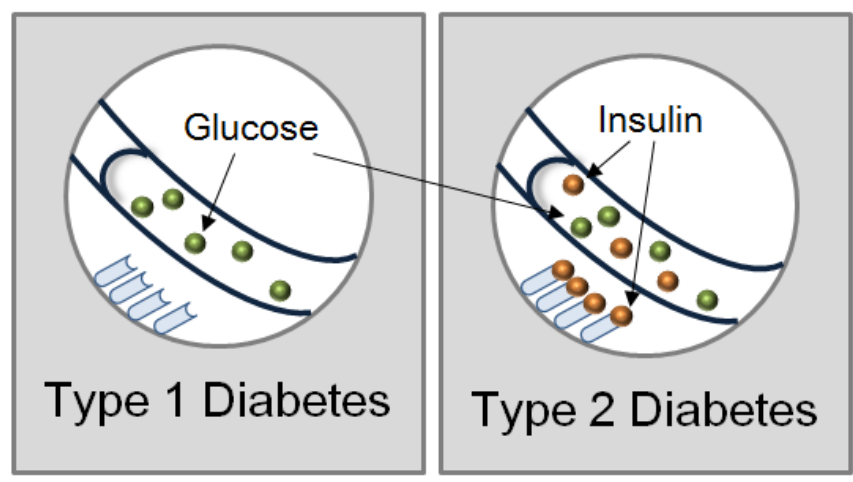

Figure 1: The key factors differentiating Type 1 and Type 2 diabetes involve the body's ability to produce or process insulin [3]. 
Type 1 diabetes can develop at any age but typically develops during childhood or adolescence. There is no known prevention method for this type of diabetes since it is caused by an unknown auto-immune reaction in which the body attacks insulinproducing cells in the pancreas. Due to this lack of insulin, individuals with Type 1 diabetes require daily insulin injections to control their glucose levels for survival. Type 1 diabetes accounts for one in 20 adults with diabetes and continues to increase amongst youth. More than 18,000 youth were diagnosed with Type 1 diabetes in 2008 and 2009, and the number continues to grow each year [2].

Type 2 diabetes can develop at any age but typically develops during adulthood. Individuals with Type 2 diabetes have trouble processing insulin, therefore eliminating cells' ability to absorb glucose for energy. They require close maintenance of glucose levels throughout the day and typically require insulin injections similar to those with Type 1 diabetes. Risk factors include being overweight, having a family history, or temporarily developing diabetes while pregnant, which are all common factors that cause one out of three people to develop Type 2 diabetes in their lifetime.

Individuals with diabetes also pay double the amount of medical costs than those without diabetes. The financial cost of diabetes in America totals $\$ 245$ billion a year including lost work and wages for people with diabetes. These medical expenses account for $\$ 176$ billion spent on maintaining blood glucose levels through at-home blood tests, doctor or hospital visits, and any insulin injections necessary [1]. The most common athome test for blood glucose levels involves pricking the skin with a needle to extract a few microliters of blood for chemical analysis with a disposable test strip. This invasive method imposes not only a financial burden for the equipment, but also a physical burden 
for the pain involved. These issues are especially problematic for seniors with diabetes who compose $25.9 \%$ of the senior population greater than 65 years old in America [1].

Test strips alone range from 40 cents to over a dollar per strip [4] which adds up to multiple dollars a day and thousands of dollars a year to properly monitor glucose levels. The test strips must also match the manufacturer of the blood glucose meter, so diabetics do not have the option to alleviate medical expenses by purchasing less expensive test strips from another company. An individual's choice in purchasing a glucose meter can also be restricted by their insurance company since they choose which glucose meter manufacturers their policies will cover. While seemingly small on an individual basis, these issues surmount to great nuisances since diabetics must inject themselves up to eight times a day [5]. An injection frequency of eight times a day means that diabetics must physically prick themselves before and after every meal if they consume four meals a day. These financial and physical burdens contribute to an increased disposition for testing blood glucose levels and cause many diabetics to avoid testing their blood glucose levels. Without proper glucose monitoring, an individual increases their risk for medical complications, such as organ failures and other potential conditions previously stated.

The underlying facts contribute to the general statement that diabetes is a major chronic disease and plagues more and more citizens every year. It is currently the $7^{\text {th }}$ leading cause of death in the United States and contributes to more deaths when combined with other health implications [2]. The availability of a non-invasive glucose monitoring device would alleviate the pain and inconveniences associated with current at-home blood tests and allow for easier maintenance of blood glucose levels. However, 
the greatest difficulty lies in creating a reliable and accurate test that also factors out contributors to measurement noise, such as skin temperature, fat percentage, and hydration levels.

\subsection{Glucose Monitoring Methods}

All forms of blood glucose sensing fall into one of two categories: invasive or minimally invasive sensing and non-invasive sensing. The majority of individuals with diabetes use invasive blood glucose at-home monitoring systems due to their high accuracy [6]. However, the circumstances are not ideal when needing multiple tests throughout the day as previously detailed. The hassle and pain involved in extracting every blood sample has sparked greater interest in non-invasive blood glucose monitoring by both researchers and biomedical companies alike.

\subsubsection{Invasive or Minimally Invasive Glucose Monitoring}

The general public with diabetes continues to use a first-generation sensing technique, the invasive finger prick test, despite the ever-advancing technology towards non-invasive glucose sensors [2]. The finger prick test determines glucose levels with a chemical reaction in which an enzyme called glucose oxidase (GOx) reacts with glucose [7]. The resulting compounds are glucuronic acid and hydrogen peroxide, which reacts with another compound to produce a colored dye. Visible light is then used to analyze the absorbance of the solution to determine blood glucose levels. Although efforts have been made to minimize the amount of test blood drawn or reduce test pain by invading a less-sensitive area of the body, the blood extraction process still remains a hassle to the everyday person. The finger prick method also exposes the body to a possible infection 
at the testing site, regardless of needle size, since the procedure involves a foreign object that invades the body.

Minimally invasive methods have also been developed to analyze interstitial fluids, such as blood plasma, for constant monitoring systems. These interstitial fluids are typically extracted through microelectromechanical systems (MEMS) implanted at a subcutaneous level [8]. One such example is the portable MiniMed Paradigm Real-Time Revel System by Medtronic [9]. The pocket-sized MiniMed system incorporates a constant blood glucose monitor and insulin pump, both implanted just below the skin. The glucose monitor provides constant glucose level readings and can alert the user of oncoming glucose lows and highs for faster treatment. An accompanying insulin pump also allows a diabetic to constantly supply their body with insulin throughout the day instead of self-injection at intermittent testing times. Constant glucose monitoring systems are most desirable because they eliminate the need for upkeep of a blood testing schedule, which is also a common deterrent for properly maintaining glucose levels. Despite their minute size, minimally invasive sensors for constant glucose monitoring still require an implantation procedure and risk potentially dangerous reactions between the device and body. An open hole on the body increases chances for possible infections and bacteria entering the monitoring site. Furthermore, constant monitors such as the MiniMed Revel system also require calibration with test strips at least once every 12 hours of operation. This is common for continuous glucose monitors since they are meant to supplement, not replace, a blood glucose meter. The MiniMed exemplifies that systems aiming to continuously monitor blood glucose levels can only alleviate some of the pain from invasive testing instead of removing it completely. 
Additionally, continuous glucose monitors are not capable of smart glucose sensing, or the ability to provide insulin when a dangerous glucose level is reached. A complete closed-loop device that both senses and maintains glucose levels would alleviate the pain and hassle associated with monitoring blood glucose. Systems with "smart" sensing capabilities such as these are difficult to incorporate due to FDA regulations; leaving biomedical companies with the option to either accurately sense blood glucose or accurately provide insulin instead of doing both. FDA regulations require at-home blood glucose monitoring systems to maintain $95 \%$ of results within a $15 \%$ range of the true value [10]. The laws are even stricter for clinical meters, of which $99 \%$ of determined values must be within $10 \%$ of the true value. These stringent regulations create difficulties in designing a system that accurately monitors glucose levels in addition to releasing insulin of the right dose and time.

\subsubsection{Non-Invasive Glucose Monitoring}

Several non-invasive methods have been developed to address the physical inconveniences of blood glucose monitoring previously stated. Whether continuously monitoring tissues or intermittently testing fluids, accuracy of these monitors still remains a factor. The following sections provide a brief overview of published non-invasive glucose monitoring methods and their success rates. 


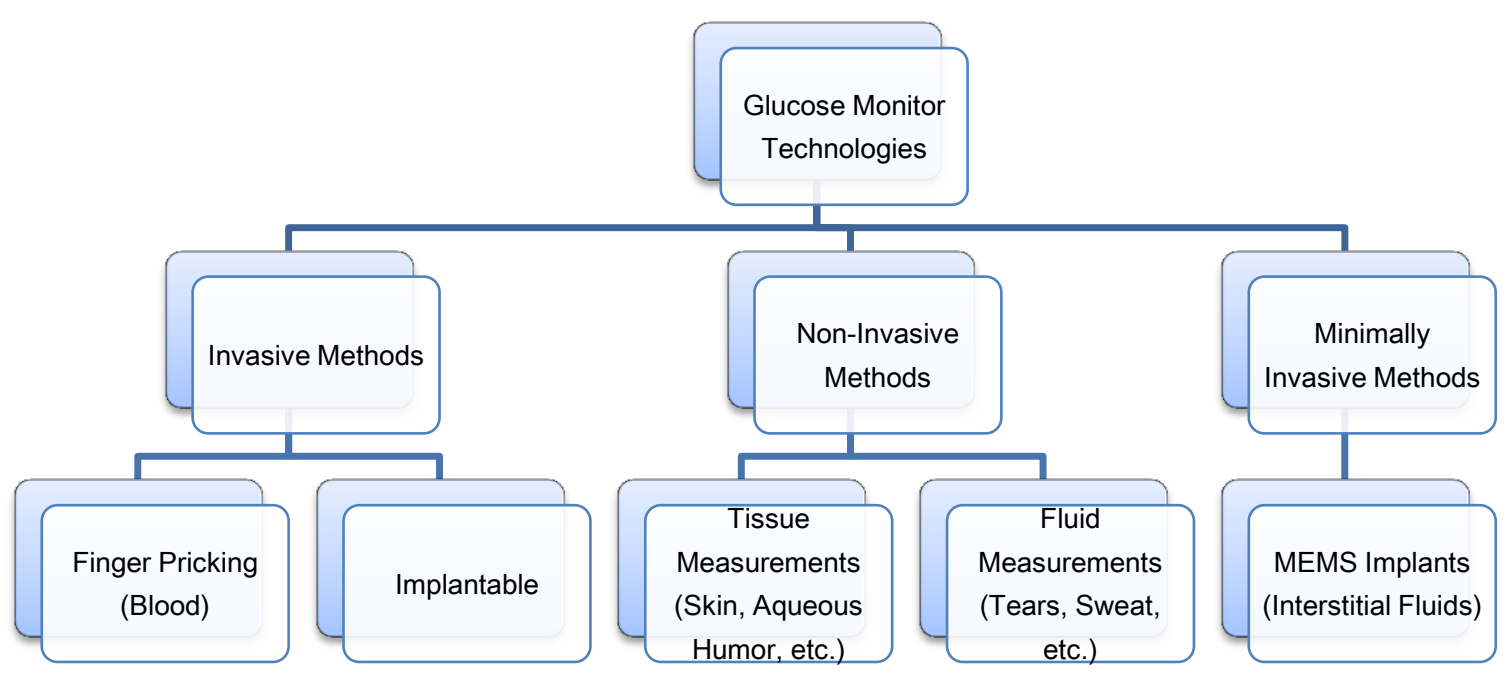

Figure 2: Summary of invasive, minimally invasive, and non-invasive blood glucose monitoring technologies.

\subsubsection{Reverse Iontophoresis}

Iontophoresis uses electrical currents in the $\mathrm{mA}$ range to deliver drugs with an electrical charge through the skin into the body. The reverse process occurs by using a potentiostat circuit with electrodes to create electro-osmotic flow. This flow creates movement of the sodium ions towards the skin's surface, which also drag the neutral glucose molecules through the skin to an iontophoretic cathode [11]. This method draws $\mu$ Mols of glucose out of the body and creates a reverse direction of movement to the original drug delivery technique. The iontophoretic cathode uses hydrogels containing the enzyme glucose oxidase (GOx) that react with the extracted glucose to create $\mathrm{H}_{2} \mathrm{O}_{2}$. The analysis circuit also uses amperometry, the method of determining ions present in a solution with electric current, to read the amount of $\mathrm{H}_{2} \mathrm{O}_{2}$ with merely nA of current.

Reverse iontophoresis can be advantageous when paired with the correct mathematical algorithms to determine glucose levels but must also account for skin temperature and perspiration. Individuals could also suffer from skin reactions to the 
potentiostat circuit which further affects the accuracies of the system. The method itself also requires time to calibrate, catalyze, and process the chemical reactions which creates a delay from current blood glucose levels to the most recent measurement. The Glu-Call from KMH Company is currently one of the best utilizations of reverse iontophoresis but has a warm up time of 70 minutes.

\subsubsection{Absorption Spectroscopy}

Upon directing light into biological tissue, the light particles undergo reflection, scattering, and transmission in a manner dependent on the chemical structure of the biological tissue. These changes create a unique response of the tissue depending on the glucose level and remain one of the most popular non-invasive detection methods. Most work already completed in this field use frequencies in the visible and near infrared ranges for a number of reasons: water does not absorb many particles at these frequencies, which creates a stronger signal for measurement, and there exist a larger number of light transducers commercially available. This frequency range also allows for particle reflection at both superficial and deep tissue layers $(1-100 \mathrm{~mm}$ for near infrared), thus narrowing the expected measurement and increasing glucose detection accuracy [12].

Dozens of companies are developing continuous glucose monitoring products based on some form of absorption spectroscopy but have yet to create a successfully accurate, reliable, and FDA-approved device for market consumption. Limitations include variations in blood pressure, body temperature, skin hydration, and triglyceride concentrations, all factors which affect the reflection and absorption of light when directed through tissue. Another significant obstacle is the difference in skin elasticity 
and thickness from person to person which also affects the light scattering response of the measurement site [13].

\subsubsection{Photoacoustic Spectroscopy}

Photoacoustic spectroscopy uses pulses of light (pico to nanosecond range) on tissue to generate heat from light particle absorption. The generated heat creates pressure differentials in the targeted tissue and therefore produces ultrasonic waves. When combined with incident wavelengths, the ultrasonic waves can be detected by piezoelectric transducers to determine optical properties of blood. These optical properties directly relate to the presence of chemical compounds such as glucose.

The drawbacks and uncertainties of photoacoustic spectroscopy remain since the detected ultrasonic waves can be affected by several other factors in tissue properties such as blood pressure, hydration, and body temperature. Another drawback is from the scattering effects of transmission through a dense material which varies for each individual. These physiological influences need to be factored out to truly have an accurate device with reproducible results for at-home use. This method has been most successful with the Aprise sensor from Glucon and proved to properly read blood sugar levels around 2007. Despite the high accuracy produced in clinical studies, the device has to undergo additional trials to pass regulations for the consumer market. 


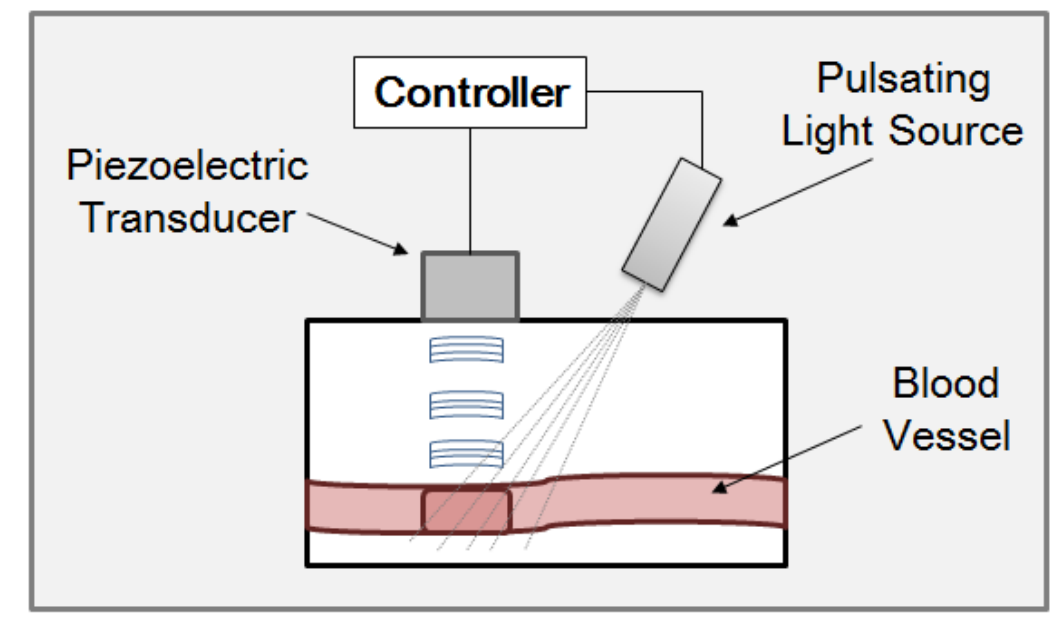

Figure 3: Photoacoustic sensing methods implement an ultrasound transducer to detect ultrasonic waves created by pulsing light on the body.

\subsubsection{Polarimetry}

When projected through optically active solutes, the amount that light rotates in the polarization plane is affected by characteristics of its path. These characteristics include thickness, temperature, and light rotation properties of the solute. Since light rotation properties of glucose have been determined, glucose detection is a feasible application for the technology. Researchers have applied this method to the aqueous humor of the eye since it does not depolarize light in the way skin does.

The aqueous humor resides beneath the cornea and above the iris and provides enough path length for a measurement as well as a short update time from the body's current glucose level. Light shines laterally through the aqueous humor without propagating through the retina. Based on the amount of polar rotation from the projected light in millidegrees, a direct glucose level can be detected at a wavelength of $633 \mathrm{~nm}$ [14]. Researchers have completed studies using a Faraday rotator and single-mode flint glass fiber to increase the optical polarization measurement sensitivity and reached a 
resolution of up to $0.55 \mathrm{mmol} / \mathrm{L}$ of glucose [15]. Despite the finely-tuned control and detection using polarimetry, the measurements can possibly be affected by ambient light exposed to the eye, temperature, and $\mathrm{pH}$ levels of the aqueous humor. Variations in corneal rotations increase the risk for eyesight damage, which makes the FDA approval process difficult for such a device.

\subsubsection{Fluorescence}

Another popular method of non-invasive glucose detection is through

fluorescence, in which a specific wavelength of light diffracts differently through a sample based on its chemical concentration. Simply put, the color of the sample changes based on its glucose concentration, and this method proves especially useful when analyzing tears. Tears are commonly used for fluorescence due to their immunity to ambient light and the relatively short lag time to blood glucose levels.

Researchers have developed disposable colorless contact lenses that sense glucose levels with additional excitation and measurement devices. Results showed a correlation between glucose levels and lens color when excited by 488nm of light [16]. Even more impressive is the creation of a colored contact lens that can display glucose levels when

they are compared to a precalibrated color test strip [17]. Unfortunately, the calibration process proves to be a difficult hurdle to overcome when combined with an uncertain resolution, lifetime, and biocompatibility of such a device.

\subsubsection{Raman Spectroscopy}

When propagating through a sample, scattered light at a given wavelength creates additional light at different wavelengths called the Raman Effect. Using lasers in the visible to mid infrared ranges of wavelength, this method proves to be more 
advantageous because the measurements are not affected by water, and the resulting wavelengths produced fall within distinct narrow bands. This method has been used on the aqueous humor of porcine eyes with an optical fiber and a $785 \mathrm{~nm}$ laser source. Although results showed a correlation, it has yet to be tested on humans. The greatest downside to Raman spectroscopy is smaller intensity of resulting wavelengths on the order of $10^{-3}$ which increases the necessary time for proper data collection in comparison to other optical methods [18]. The greatest danger common to all optical sensing methods also applies in the stability of the laser source when measuring from the eye and the potential harm to the user's vision.

\subsubsection{Metabolic Heat Conformation}

Using thermal and optical measurements of physiological parameters, blood glucose levels can be detected with a strong correlation. These targeted parameters include thermal generation, blood flow rate, hemoglobin, and oxyhemoglobin since glucose directly affects blood heat, flow, and oxygenation [19]. Metabolic heat conformation involves excitation from multiple LEDs in the near infrared and visible wavelength ranges for optical and thermal measurements. Optical reflections are measured, as well as tissue radiation, convection, and thermal transfer to determine heat and blood flow changes. A function based on the amount of heat generated by these LEDs, plus the determined blood flow rate and oxygenation determines glucose levels.

Researchers have produced results with a correlation coefficient of 0.91 when measurements were combined with the proper statistical analyses and mathematical procedures. Although the strong correlation coefficient proves this is a reliable method, it can still be affected by changes in environmental conditions. Metabolic heat 
conformation is therefore typically used in combination with another measurement technique for increased accuracy.

\subsubsection{Thermal Emission Spectroscopy}

Thermal emission spectroscopy focuses on the fact that mid-infrared waves naturally emit from the body, especially the tympanic membrane. These spectral emissions vary based on the concentration of glucose in the blood and therefore provide a factor for measurement simply using thermometers. Tympanic membrane (eardrum) thermometers, typically found in the hospital and home settings, gather temperature information by determining total energy across a wide spectral range of emissions from the body. Upon adding the wavelengths specifically for glucose (between $8 \mu \mathrm{m}$ and $14 \mu \mathrm{m})$ to the measurement process, glucose levels can be determined.

The advantages to this method are significantly outstanding because the eardrum has the same blood supply as the temperature-regulating hypothalamus, which also controls the physiological processes involving insulin. The distance to this blood supply is much less than the thickness of skin or oral mucosa sites and therefore reduces the lag time of blood glucose updates at the measurement location. A prototype was created and proved fair accuracy with a correlation coefficient of 0.87 based on a calibration model with a mean error of $0.638 \mathrm{mmol} / \mathrm{L}$ [20]. The results lacked greater accuracy due to noise caused by body movements, variation of body temperature, and ambient temperature.

\subsubsection{Ultrasound}

Ultrasound methods use techniques similar to that of reverse iontophoresis in which the skin is excited with a specific type of circuit to draw glucose to the surface. In this case, the excitation circuit is a piezoelectric transducer at $20 \mathrm{kHz}$ which increases 
skin permeability to glucose after a given time. Using a similar amperometric electrode and glucose oxidase hydrogel, the amount of glucose extracted from interstitial fluid can determine blood glucose levels.

A study was completed on which rats with diabetes were tested, and the results were compared to a commercial glucose meter [21]. The results showed the existence of a correlation between the measured and true values; however the accuracy was still questionable for FDA-approved glucose detection methods. Again, noise inducing factors include skin temperature and perspiration which were unaccounted for in the study on rats.

\subsubsection{Bioimpedance Spectroscopy}

Technologies based on bioimpedance spectroscopy typically implement an LC resonant circuit coupled to the skin in combination with an impedance measuring circuit. The skin acts as the dielectric for the capacitor, and the blood membranes beneath the skin therefore change the impedance detected. Studies have shown that glucose affects blood conductivity which further influences the polarity and impedance of blood cell membranes. Detecting at a frequency that is low enough to sense this polarity and high enough to avoid signal dispersion in tissue, the range between $1 \mathrm{MHz}$ and $200 \mathrm{MHz}$ was chosen [22].

These researchers were able to develop a commercial device called the Pendra by Pendragon, which imitated a wrist watch form factor. The device was approved in 2003 by the Conformité Européene (CE) and on the market for a short time before reliability testing revealed its inconsistencies across users. It was found that the Pendra only worked for customers with a local skin dielectric with minimal resonant frequency. The 
greatest disadvantage to this device was the 60 minutes of rest time necessary for the device to calibrate itself before beginning measurements.

\subsubsection{Electromagnetic Sensing}

Electromagnetic sensing parallels bioimpedance spectroscopy. Instead of sensing impedance through current, electromagnetic methods sense dielectric parameters of blood through electromagnetic coupling [23]. The strength of electromagnetic coupling between two inductors is directly affected by the dielectric properties of the material between the inductors. Dielectric property, also called relative permittivity, reflects how easily a material can become charged by an imposed electric field [24]. At blood glucose concentrations at and lower than $150 \mathrm{mg} / \mathrm{dL}$, glucose has been shown to inversely affect the dielectric constant and therefore make blood more susceptible to imposed electric fields [25]. Because of this effect, two electromagnetically coupled inductors could be placed across a blood supply. The output voltage of the secondary inductor would change depending on the blood's dielectric constant when the primary inductor is excited with a specific voltage and frequency. Therefore the ratio between the output RMS voltage and input RMS voltage provides a direct estimation of the blood glucose levels.

The main hindrance on electromagnetically sensing blood glucose levels with high resolution lies in the optimal frequency at which to excite the transmitting inductor. Previous research shows that the target frequency for electromagnetic sensing falls in the 2.4-2.9 $\mathrm{MHz}$ range [12] due to the absorptive tendencies of tissue at higher frequencies [26]. However, the optimal frequency also depends on the tissue's temperature as well as the distance between the two inductors. Since biological tissue exponentially decays the electromagnetic coupling of two inductors, the distance between the inductors greatly 
affects the output voltage. These drawbacks affect the reliability and reproducibility of this method and stand in the way of market-ready devices for electromagnetic glucose sensing.

\subsection{Problem Definition}

Since invasive glucose sensing methods impose physical and financial burdens on those with diabetes, the need for a reliable non-invasive glucose sensing technique is undeniably present. While other non-invasive sensing methods exist, the majority of signal-based systems relevant to the scope of this thesis have been detailed. The descriptions have provided both positive and negative aspects of each technology and argue the need for new glucose sensing methods. In summary, most of the unobtrusive glucose sensing methods described contain a fatal flaw to prevent their success. Some examples are absorption spectroscopy and polarimetry, which lack portability because they require large and bulky equipment to carry out such a measurement. Potentially portable methods, such as photoacoustic spectroscopy or metabolic heat conformation, require great power sources to provide energy to multiple components and tremendously affect the size and weight of these designs. Techniques that do not require large power sources add up in cost due to special chemicals and materials for the manufacturing process, similar to those used for fluorescence, reverse iontophoresis, and ultrasonic sensing methods. The issue of accuracy, consistency, and reliability still present themselves in these non-invasive methods, and such combined obstacles provide diabetics with reason to use first-generation invasive techniques. 


\section{Chapter 2: Background}

Signal-based glucose sensing methods offer great promise for non-invasive monitors in the near future. Especially considering how glucose levels change more frequently throughout the day than other blood components, signal-based sensors are optimal for quickly reading and tracking blood glucose levels. Of the methods listed in Chapter 1, electromagnetic sensing techniques have proven their potential for great success due to recent developments in the medical field.

\subsection{Potential New Methods for Electromagnetic Sensing Technologies}

Technology implementing electromagnetic wave propagation has also been found to benefit the need for wirelessly powering deeply implanted devices, such as pacemakers. Battery sizes impose a size constraint on medical implants and have yet to catch up to the miniaturization of component sizes. Batteries constitute the majority of the size of medically implanted devices and can also require replacement via medical surgery once their energy has depleted. Previous attempts at wireless transmission focused on near field coil coupling due to the lower target frequency, but researchers at Stanford University have discovered the benefits of using higher frequency with receivers in the midfield range for optimal power transfer efficiency [26], [27], [28].

\subsubsection{Determining Optimal Frequency}

As stated previously, target frequencies of electromagnetic coupling were in the megahertz range due to the exponential decay of electromagnetic fields in tissue. This decay relates to the diffusion equation approximating Maxwell's equations that govern the properties of electromagnetic fields in good conductors. The equation reveals that the diffusion length is inversely proportional to the square root of frequency and thus makes 
it ideal to use lower frequencies for greater propagation. However, Poon et al.

discovered that tissue is a low-loss dielectric more than it is a good conductor [26]. Low loss dielectrics have greater displacement current, which significantly increases power transfer at high frequencies. The following sections review the theory of power transfer efficiency, the use of inductive coupling for power transfer, and the new optimal frequency in the low-gigahertz range.

\subsubsection{Power Transfer Efficiency}

Power transfer efficiency between an inductively coupled source and receiver depends on total tissue absorption. Before regarding losses caused by tissue properties, a general comprehension of power transfer efficiency in weak, inductively coupled coils should be understood. To further analyze power transfer efficiency, the source and receiver can be modeled as a two-port network with the following linear equations:

$$
\begin{aligned}
& V_{1}=Z_{11} I_{1}+Z_{12} I_{2} \\
& V_{2}=Z_{21} I_{1}+Z_{22} I_{2}
\end{aligned}
$$

Subscript 1 denotes the source, and subscript 2 denotes the receiver in these equations.

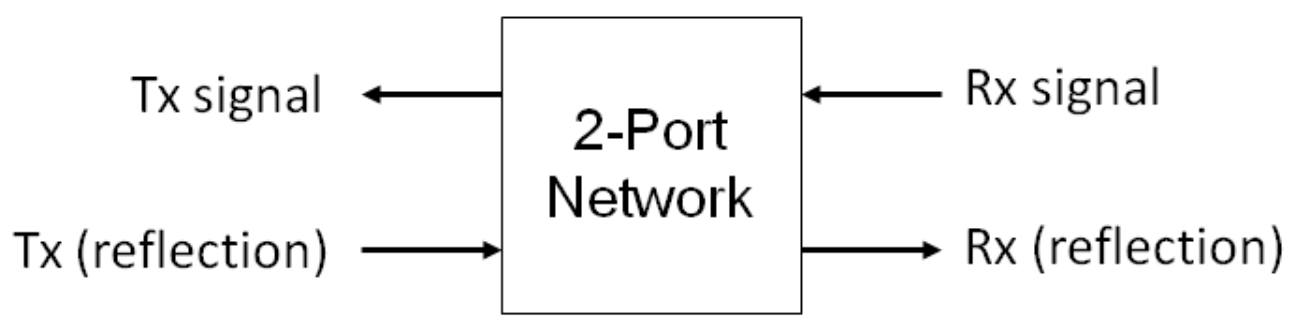

Figure 4: Block diagram of a 2-port model displaying relations between the source and receiver that are necessary for understanding inductively coupled coils.

Determining the real part of the impedance parameters identifies the loss in the system and reveals that $\operatorname{Re}\left\{\mathrm{VI}^{*}\right\} / 2$ gives the dissipated power. With a passive receiver and a 
load impedance of $Z_{L}$, received current can be reduced to $I_{2}=-V_{2} / Z_{L}$. Reciprocity of the system also determines that $Z_{12}=Z_{21}$. Replacing these variables with $I_{2} / I_{1}=$ $Z_{21} /\left(Z_{22}+Z_{L}\right)$ from the determined parameters, the power transfer efficiency is described as

$$
\eta=\frac{R_{L}\left|I_{2}\right|^{2}}{R_{1}\left|I_{1}\right|^{2}+\left(R_{2}+R_{L}\right)\left|I_{2}\right|^{2}+2 R_{21} \operatorname{Re}\left\{I_{1}^{*} I_{2}\right\}}
$$

In addition, the optimal load reactance when maximizing the efficiency with respect to load impedance is given by

$$
X_{\text {L.opt }}=-X_{2}\left(1-\frac{X_{21} R_{21}}{X_{2} R_{1}}\right)
$$

When the load resonates with the receiver, $X_{L, o p t} \approx-X_{2}$, the optimal load resistance reduces to

$$
R_{\text {L.opt }}=R_{2} \sqrt{1-\frac{R e\left\{Z_{21}^{2}\right\}}{R_{2} R_{1}}}
$$

and this equation can be used to determine the new power transfer efficiency. By setting $Z_{L, o p t}=R_{L, o p t}+i X_{L, o p t}$, the new power transfer efficiency follows the equation

$$
\eta\left(Z_{L, o p t}\right)=\frac{\frac{\left|Z_{21}\right|^{2}}{R_{1} R_{2}}}{2-\frac{R e\left\{Z_{21}^{2}\right\}}{R_{1} R_{2}}+2 \sqrt{1-\frac{R e\left\{Z_{21}^{2}\right\}}{R_{2} R_{1}}}}
$$

Additional variables may further be eliminated since the receiver is assumed to be in the weakly coupled regime where $\left|Z_{21}\right|^{2} /\left(R_{1} R_{2}\right) \ll<1$. This provides a new efficiency equation of

$$
\eta \approx \frac{\left|Z_{21}\right|^{2}}{R_{1}} \frac{R_{L}}{\left|Z_{2}+Z_{L}\right|^{2}}
$$


Maximizing $\eta$ can be achieved through conjugate matching when $Z_{L, o p t}=R_{2}-i X_{2}=Z_{22}{ }^{*}$, thus reducing the coupling efficiency to

$$
\eta_{c}=\eta\left(Z_{L, o p t}\right)=\frac{\left|Z_{21}\right|^{2}}{4 R_{1} R_{2}}
$$

This relation provides the optimal ratio between received power to the input power based on the two-port impedances and resistances. Now that the power transfer efficiency has been determined for an assumption of weakly coupled coils, further analysis on inductive coupling in multi-layered tissue is necessary to find the optimal operating frequency.

\subsubsection{Inductive Coupling}

Inductive coupling for medical devices typically follows an analysis assuming slow-changing fields. The slow-changing fields are due to the lower frequencies common in these applications. A lower frequency range allows for the quasi-static approximation or a disregard of the displacement current from Maxwell's equations. This approximation reduces the effective magnetic field, $\mathbf{H}$, to become mostly reactive and yields the following output voltage:

$$
V_{2}=-i \omega M I_{1}+\left(i \omega L_{2}+R_{2}\right) I_{2}
$$

The equation includes $\mathrm{M}$ as the mutual inductance, $\mathrm{L}$ as the self-inductance, and $\mathrm{R}$ as the lumped resistance of the receiver coils. The coupling coefficient can also be found to be $k=M / \sqrt{L_{1} L_{2}}$, a value independent of the propagating tissue and solely dependent on the coils' properties.

Another result of the quasi-static approximation is the simplification of the optimal load to 


$$
Z_{L, o p t}=R_{2} \sqrt{1-\frac{R e\left\{Z_{21}^{2}\right\}}{R_{1} R_{2}}-i X_{2}}
$$

This describes that the optimal power efficiency must actually follow a general rule of $\left|\mathrm{Z}_{21}\right|^{2} / \mathrm{R}_{1} \mathrm{R}_{2} \gg>1$, contrary to the values previously determined. Following this rule aids in impedance matching to the optimal load and therefore achieves the greatest power efficiency between multi-layered, low-loss tissues.

\subsubsection{Higher Frequencies}

When designing for impedance matching to the optimal load, the coupled coils must be placed several centimeters apart to maintain the $\left|Z_{21}\right|^{2} / R_{1} R_{2}>>1$ rule. This design rule places the receiver and the source within a wavelength of each other, also known as the midfield. Midfield antennas operate in the low-gigahertz range given the distances described, thus increasing the target frequency higher than the initial megahertz range. The midfield also uses both reactive and radiative modes to strengthen the coupling between the source and receiver and allows for a receiver coil in the millimeterdiameter range. Maximizing the coupling parameter in the midfield can produce an optimal frequency that creates a power efficiency independent of the coil or matching considerations. Maximizing the coupling parameter involves finding the magnetic dipole moment for a coil area $\mathrm{A}_{\mathrm{r}}$ in the direction $\mathrm{N}$ as follows:

$$
M=i \omega \mu_{0} A_{r} I \delta\left(r-r_{0}\right) N
$$

This magnetic dipole moment therefore determines the coupling parameter as

$$
\gamma=\frac{\left|V_{O C}\right|^{2}}{2 P_{1}}=\frac{\omega \mu_{0}^{2}\left|\boldsymbol{H}_{1}\left(r_{f}\right) N\right|^{2}}{\int \operatorname{Im} \epsilon(r)\left|E_{1}(r)\right|^{2} d r}
$$


The coupling parameter shows that increasing $\omega$ increases the induced $\mathrm{V}_{\mathrm{oc}}$, but becomes limited by the existing, albeit low, dielectric losses of the propagating material. These losses can be characterized by the tissue conductivity and dielectric relaxation. The Debye relaxation model demonstrates both the characteristics of dielectric loss with the following equation:

$$
\epsilon_{r}(\omega)=\epsilon_{\infty}+\frac{\epsilon_{r}-\epsilon_{\infty}}{1-i \omega \tau}+i \frac{\sigma}{\omega \epsilon_{0}}
$$

The dielectric permittivity at the infinite field is defined by the dielectric permittivity of the material in which the receiver resides. All dielectric permittivities and conductances depend on the type of tissue, such as blood, bone, or fat as determined in [29].

Based on these parameters, two angle orientations $\left(0^{\circ}\right.$ and $\left.90^{\circ}\right)$ for the receiver coil were researched for determining the optimal operating frequency [28]. Green's assumptions were used to determine the sum of vector multipoles of the dielectric permittivities at each frequency. The assumptions produced an asymptotic expression that modeled the generic equation for optimal frequency

$$
f_{\text {opt }} \approx \frac{1}{2 \pi} \sqrt{\frac{c \sqrt{\epsilon_{r 0}}}{\tau d\left(\epsilon_{r 0}-\epsilon_{\infty}\right)}}
$$

This optimal frequency approximation reveals that midfield range receivers require higher operating frequencies in the low-gigahertz range. A possible benefit of higher frequencies in the gigahertz range includes greater open-circuit voltages for potentially faster wireless power transfer. Considering the distance to the sensor and the specific relative permittivities of skin, fat, muscle, and bone combined, a frequency of 1.6 GHz was estimated in [27]. This computation accounted for all estimated thicknesses of biological layers in the chest of the human body. 


\subsection{Source Realization}

After determining the optimal frequency, the proper source must be designed to provide the greatest power to a receiver resonating at that frequency and at a given distance. Due to the nature of wave propagation in tissue, the source must optimize radiation concentration to provide maximum power transfer. The radiation concentration can be determined by the in-plane electric current density, $\mathbf{J}$, which faces complexities at boundaries between two different layers of material. Accounting for these complexities and determining the necessary electric current density for highly efficient power transfer yields the radiation pattern of the source required to achieve said density. Therefore, determining the radiation pattern based on the desired electromagnetic fields defines the source design. This governing radiation pattern also implies that the electric current density is the primary parameter that bounds the power transfer efficiency of the source and receiver.

Ho et al. [27] determined that the planar current density patterns for optimal power transfer of a source external to the body and a receiver internal to the body are similar to those shown in Figure 5. The radiating shapes were realized in a slot array antenna shape, as shown in Figure 7, with each slot producing constructive interference to propagate multiple centimeters into the body. Simulations were completed to show that the constructive interference from the source's magnetic fields altered the receiver's dipole moment to reach greater amounts of power, thus creating highly efficient power transfer. 

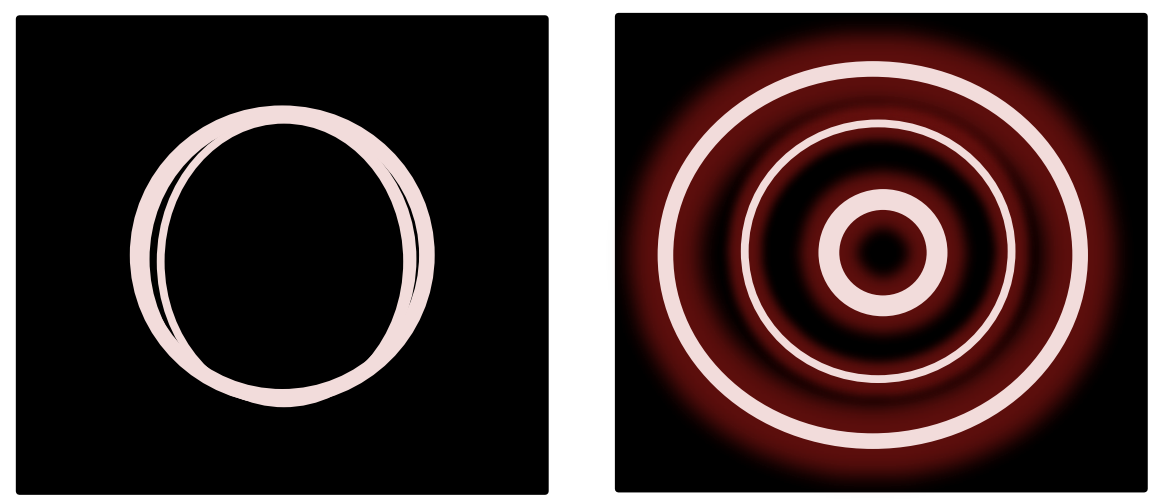

Figure 5: Electric current density realizations (left: source, right: receiver) for necessary field propagations through multiple layers of biological materials.

\subsection{Overall Results from Ho et al. [27] and Kim et al. [30]}

The resulting design features a slot array antenna that, when positioned just above the skin, can wirelessly provide milliwatts of power to microimplants greater than $5 \mathrm{~cm}$ within the human body [27]. Figure 6 details the design originating from Kim et al. [30] for the midfield wireless power transmission. A key successful attribute is the antenna's ability to propagate through multiple layers of biological matter, such as skin, fat, muscle, and bone while maintaining effective current density at the receiver. This attribute increases the coupling to a receiver implanted centimeters deep in the body and provides significant wireless power that has rarely been seen before. 

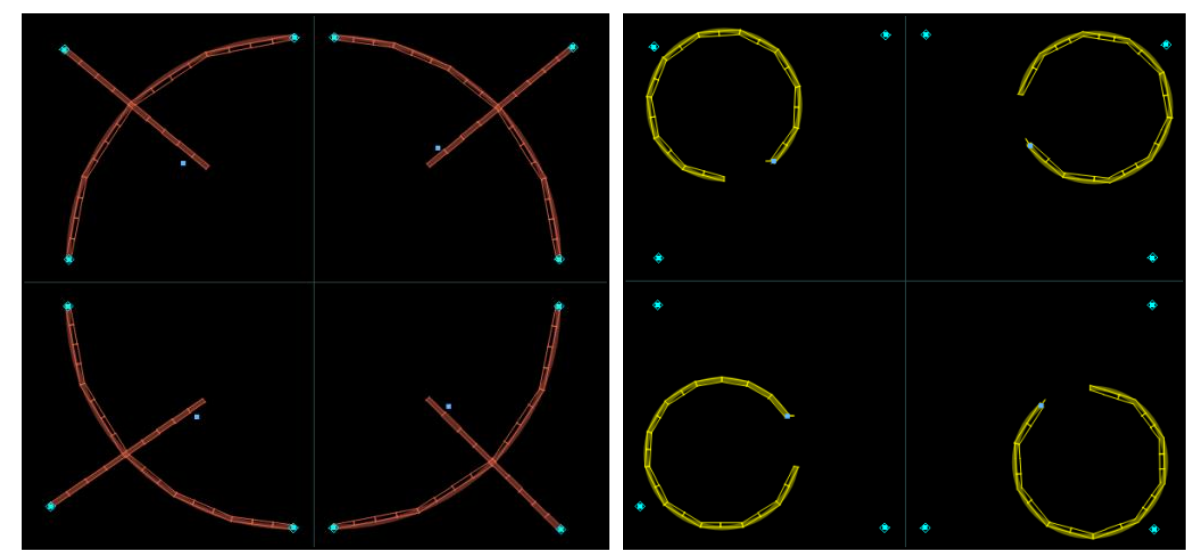

Figure 6: Slot array layout (left) and microstrip array (right) realized by Kim et al. [30] for maximum power transfer through greater than $5 \mathrm{~cm}$ of biological tissue. The microstrip layer marks the four input ports by the overlapping blue dots.

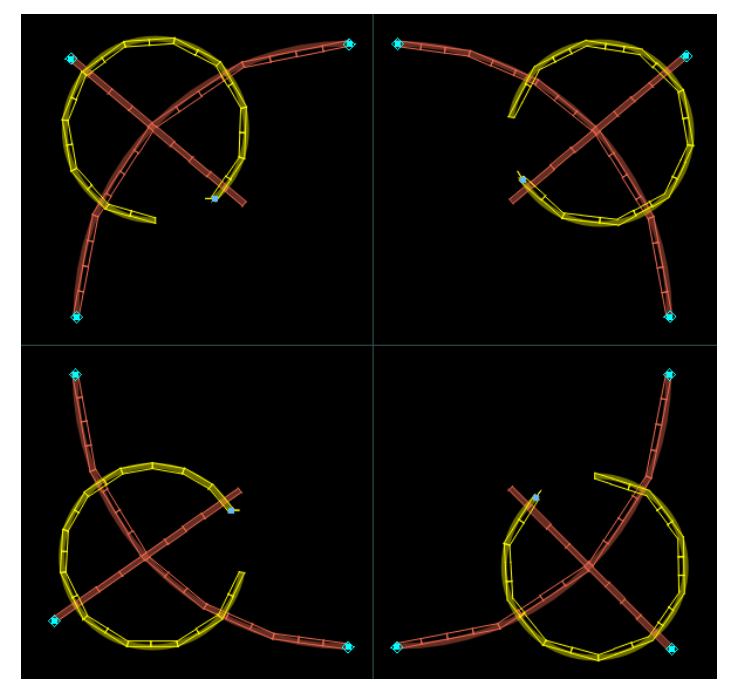

Figure 7: Image of slot array antenna with microstrip layer (yellow) superimposed on slot layer (red) designed by Kim et al [30].

Another outstanding feature is the ability to direct the antenna's output to areas much smaller than the operational wavelength. Depending on the phase of the four input ports, the region within the body with the strongest fields can be controlled to an angle between 45 and 90 degrees from the plane of the antenna. Figure 8 shows three possible 
angles of directed output field from the source, through an air gap, and to an implanted receiver in the body. This directionality aids in producing results with greater reliability and consistency by stabilizing the output field despite variations in tissue thickness.

Propagating electromagnetic waves through multiple layers while maintaining directional precision increases possibilities for additional antenna purposes, specifically electromagnetic glucose sensing.

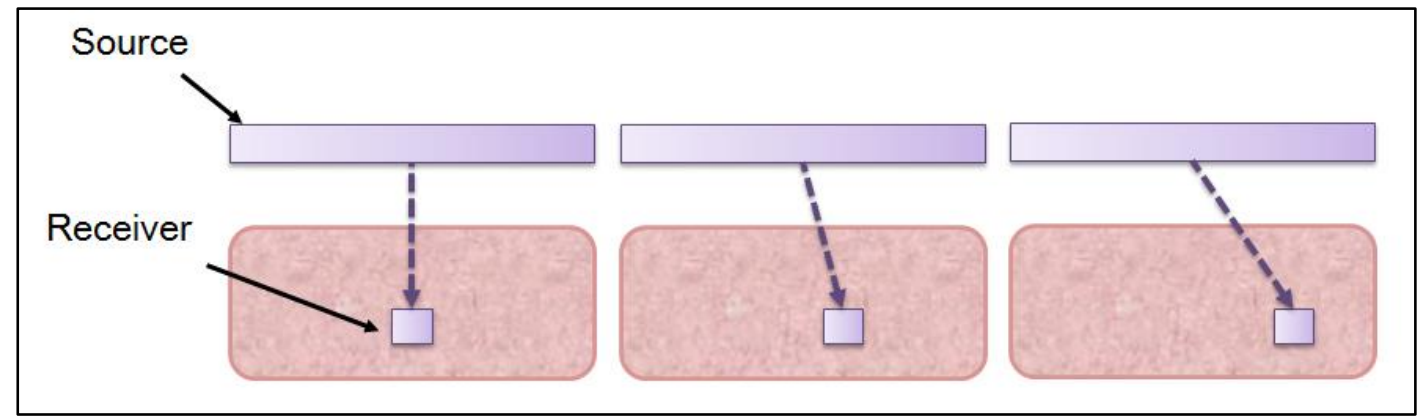

Figure 8: The antenna can produce different angles of output direction based on the phases of the four inputs.

\subsection{Safety Measurements}

Propagating electromagnetic waves through biological tissue imposes a safety hazard to the patient under test. Whether it is for medical devices or commercial electronics, harmful effects of radio frequency waves include thermal damage to the tissue propagating said waves. Thermal damage occurs when the tissue cannot dissipate the energy introduced by a radio frequency source, similar to the way that microwave ovens cook food [31]. To prevent such harmful reactions, devices operating from $3 \mathrm{kHz}$ to $200 \mathrm{GHz}$ require strict operating regulations. The IEEE Standard for Safety Levels with Respect to Human Exposure to Radio Frequency Electromagnetic Fields has defined the stringent regulations necessary for high-frequency devices interfacing to humans. 
The IEEE has defined a safety threshold of $10 \mathrm{~W} / \mathrm{kg}$ averaged over 10 grams of tissue in a controlled environment [32], therefore any excess energy beyond this poses a danger to the patient. Ho et al. completed regulatory compliance measurements of their design by analyzing the amount of energy dissipated into the tissue between the source and receiver. The regulatory compliance testing measured the spatial distribution of absorbed power throughout liquids mimicking tissue from the head and body. Results revealed that absorbed energy fell far below the safety threshold defined by IEEE. When coupling $500 \mathrm{~mW}$ to the receiver, the 10 -gram averaged specific absorption rate (SAR) never exceeded $0.89 \mathrm{~W} / \mathrm{kg}$ in the body-mimicking liquid and $1.17 \mathrm{~W} / \mathrm{kg}$ in the headmimicking liquid. The source was also able to provide $195 \mu \mathrm{W}$ and $200 \mu \mathrm{W}$ to the heart and brain, respectively, at these absorption rates [27]. Further testing also proved that the power transfer through tissue would not have a significant effect on core body temperatures due to low body-averaged absorption and localized distribution.

\subsection{An Application for Glucose Sensing}

When aiming to create a solution for non-invasively detecting blood glucose levels without additional materials for chemical analysis, electromagnetic methods have great potential for highly accurate monitoring. With the recent discoveries by Ho et al. [27], electromagnetic glucose sensing methods prove to be much more feasible with controlled directionality and stronger coupling from a midfield antenna source. Such an antenna allows for more consistent readings of expected output power at a specific location due to the increased coupling strength.

As previously described, the effect of glucose on dielectric permittivity of blood can affect the amount of power received from a coupled transmitter. To verify the 
change in electrical properties of glucose based on frequency, a simulation to model the absorption of glucose across a range of frequencies was completed. EMPro, a 3dimensional electricity and magnetism simulation software, was used to model the response of two microstrip patch antennas separated by a $1 \mathrm{~cm}$ air gap and a $5 \mathrm{~cm}$ thick block of glucose-dependent blood plasma. The microstrip patch antennas were obtained from the example projects provided by EMPro and incorporated probe-fed microstrip patches on a $0.794 \mathrm{~mm}$ thick Duroid substrate. Since the electrical properties of glucose in blood plasma change with frequency, a three-pole Debye-Drude model was implemented for the plasma. This three-pole model was found to be the most accurate parameterization of glucose in blood plasma across $500 \mathrm{MHz}$ to $20 \mathrm{GHz}$ [33], and its parameters are summarized in Table 1. Figure 9 shows the physical setup of the 3-D simulation, with two identical probe-fed microstrip patch antennas on both sides of the simulation volume.

Table 1: Three-pole Debye-Drude model determined for glucose in blood plasma.

\begin{tabular}{|c|c|}
\hline Parameter & Three-Pole Model \\
\hline Conductivity [S/m] & 0.28 \\
\hline Infinite Freq. Relative Permittivity & 7.5 \\
\hline Static Relative Permittivity 1 & 63.33 \\
\hline Relaxation Time 1 [ps] & 12.12 \\
\hline Static Relative Permittivity 2 & 3632.97 \\
\hline Relaxation Time 2 [ns] & 172.98 \\
\hline Static Relative Permittivity 3 & 256744 \\
\hline Relaxation Time 3 [us] & 156.74 \\
\hline
\end{tabular}




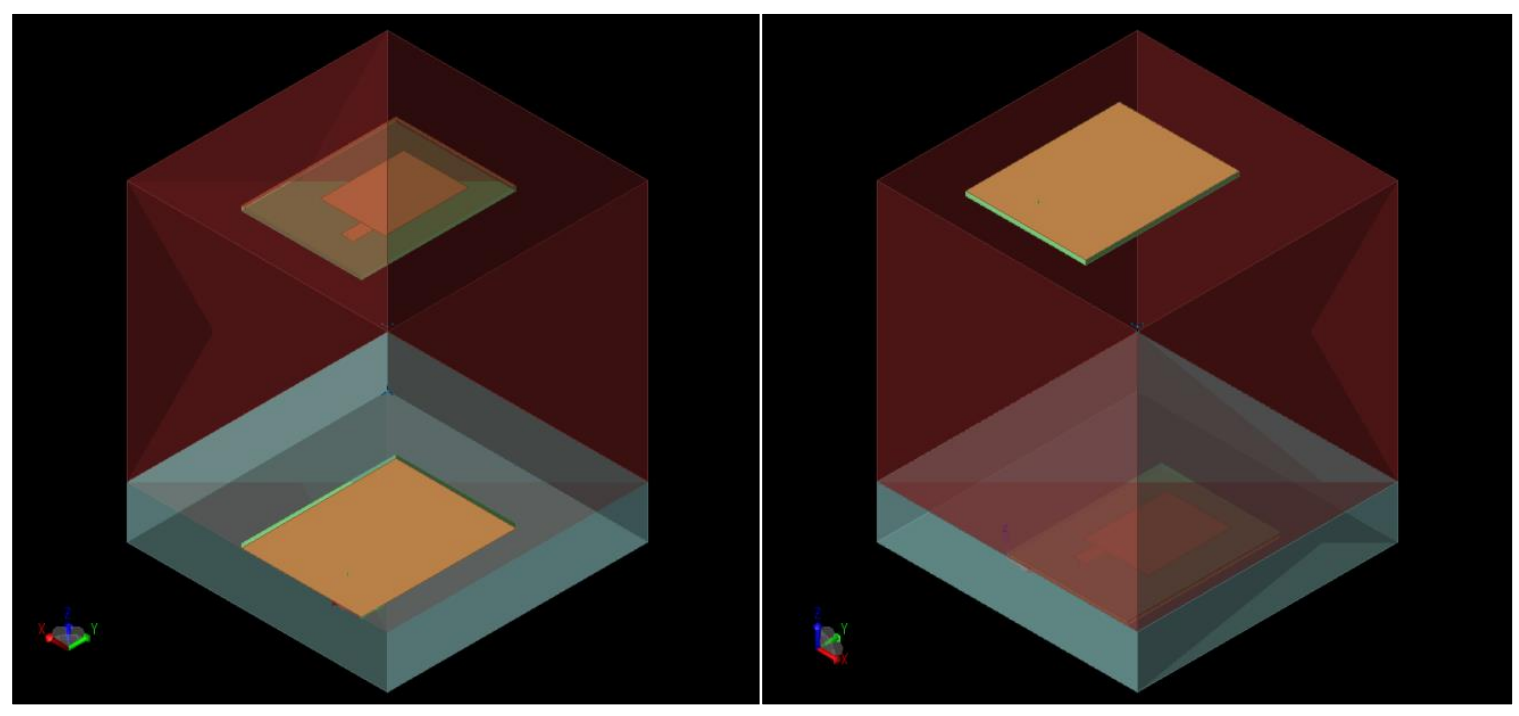

Figure 9: The 3-D simulation model of two microstrip patch antennas separated by a 1 $\mathrm{cm}$ air gap and $5 \mathrm{~cm}$ thick block of glucose was modeled to determine the optimal operating frequency for a glucose sensor.

The simulation was swept across $200 \mathrm{MHz}$ to $3 \mathrm{GHz}$ to determine the amount of energy transferred from one patch antenna to the other. The ultimate objective in completing this simulation was to determine the frequency at which glucose absorbs the most energy, which would provide the frequency with highest sensitivity for glucose sensing. Figure 10 shows the response of the receiving antenna, labeled as Port 2, in relation to the transmitting antenna, labeled as Port 1 , when Port 1 was sourced with a 1 V sinusoidal wave. The smallest magnitude detected by the receiving patch antenna was $-74.97 \mathrm{~dB}$ at $1.6 \mathrm{GHz}$, thus representing a significant loss in energy due to the natural absorption properties of glucose. Therefore, $1.6 \mathrm{GHz}$ was maintained as the optimal operating frequency of the glucose sensor. 


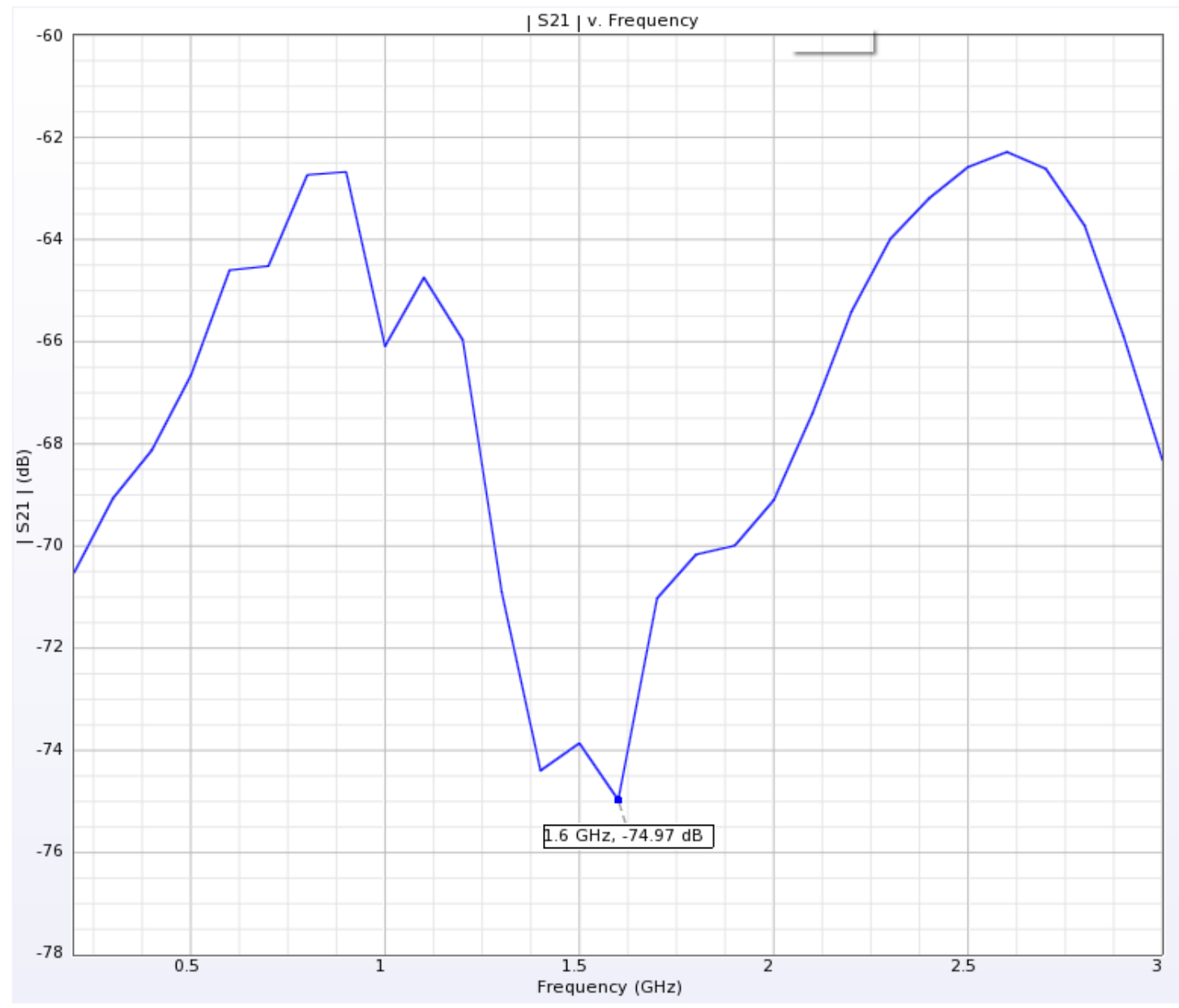

Figure 10: Based on EMPro simulations through $5 \mathrm{~cm}$ of glucose, the greatest absorption of energy occurred at $1.6 \mathrm{GHz}$.

The estimated relative permittivity for blood of varying glucose concentrations at 1.6 GHz was found in [25] and confirmed the inverse relationship between the two parameters. To further demonstrate the relation between blood glucose and received power from an electromagnetically coupled source, a simple design was simulated in a radio-frequency design software called Advanced Design System (ADS). The simulations were completed with a single slot design originating from Ho et al. that propagated into $1 \mathrm{~cm}$ of air and $4 \mathrm{~mm}$ of blood. The single input source was excited with a $1 \mathrm{~V}$ sinusoidal wave with no phase shift, and the dielectric constant of blood was 
changed to match the values estimated from [25]. Measurements of maximum radiating power were gathered from far field patterns in plane with no polar or rotational angle. Table 2 summarizes the information gathered from the simulations and the resulting relation between glucose concentration and radiated power.

Table 2: Relation between blood glucose concentration, blood relative permittivity, and the maximum radiated power as measured by ADS.

\begin{tabular}{|c|c|c|}
\hline Glucose Concentration [mg/dL] & Relative Permittivity & Power [W/Sterad] \\
\hline 134 & 59.738 & $4.77 \mathrm{E}-04$ \\
\hline 95 & 63 & $4.83 \mathrm{E}-04$ \\
\hline 72 & 65 & $4.92 \mathrm{E}-04$ \\
\hline
\end{tabular}

The trend in maximum power displays the inverse relationship between blood glucose concentration and radiated power, as graphically presented in Figure 11. Finite element analysis methods also simulated in ADS revealed the magnitude of the current density in the 4 millimeter layer of blood. Figure 5 shows the simulated current densities of blood stimulated by a single slot antenna source. The color gradient from cool to warm displays stronger current densities and increased power propagation with decreasing levels of blood glucose. Applying this midfield antenna design with knowledge of the relation between blood glucose and received power offers a potential new method for non-invasive glucose sensing. 


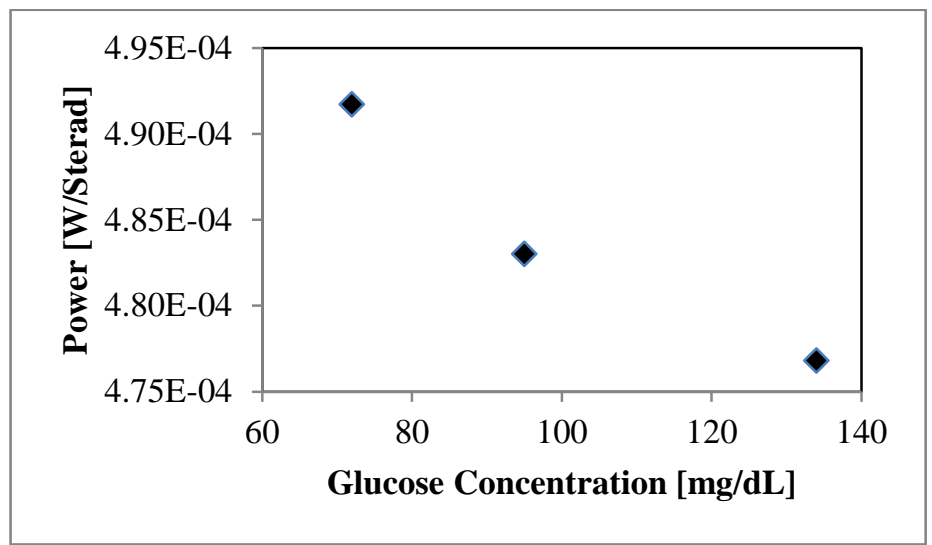

Figure 11: As previously stated, increased blood glucose concentration inversely affects the dielectric permittivity of blood and therefore decreased radiated power received.

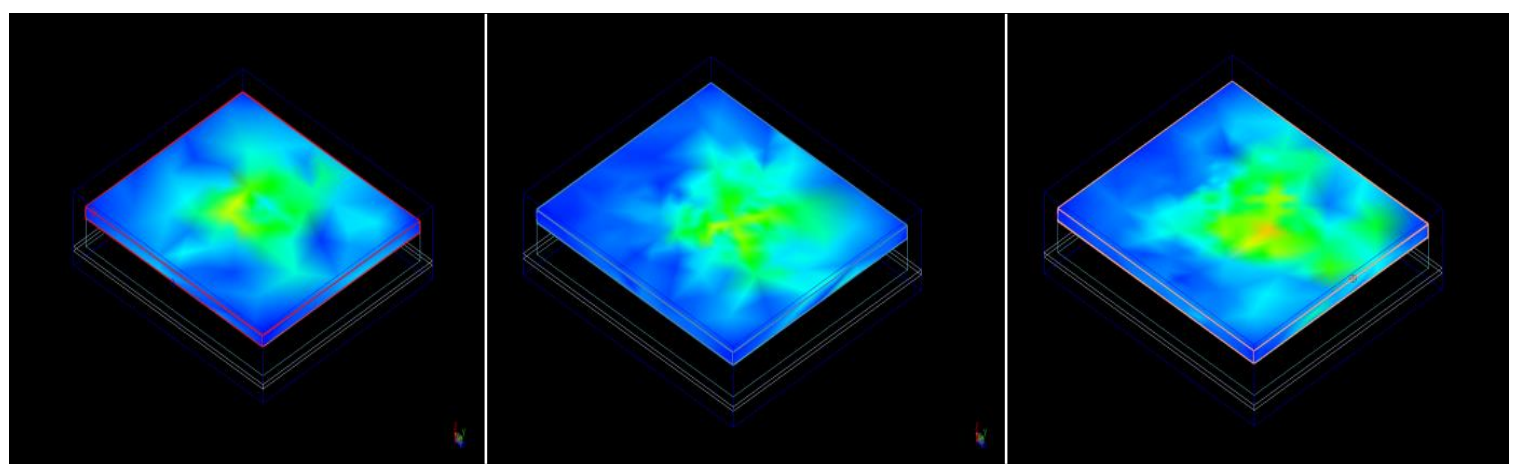

Figure 12: Current density of simulated blood glucose concentrations of 134, 95, and 72 $\mathrm{mg} / \mathrm{dL}$ respectively.

An ideal location for this test is the biceps brachii due to its ease of access. With further simulations from ADS, an expected power value can be calculated for a full slot array antenna transmitter propagating electromagnetic waves to a coil receiver placed on the other side of the biceps brachii. Figure 13 illustrates a proposed testing setup with the source directed to a receiver angled about 45 degrees through the biceps. 


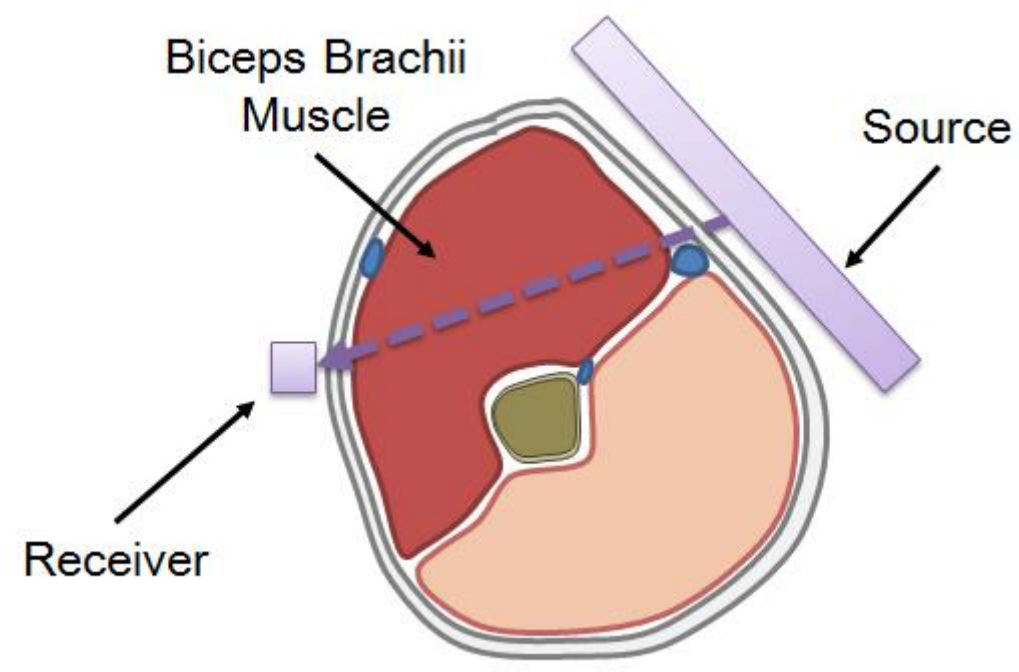

Figure 13: Cross-section of the upper arm and targeted position of the slot array antenna source and receiver with angled wave propagation intensity.

Based on the completed simulations, the absorption of glucose was the dominating cause of signal attenuation at $1.6 \mathrm{GHz}$. When combined with Ho et al.'s midfield antenna that easily transmits through tissue, this strengthens the confidence in using the antenna to sense glucose since attenuation would be caused by changes in glucose instead of changes in other physiological parameters. One example of a changing physiological parameter is water, which varies throughout the day based on the subject's hydration level. However, water does not absorb energy at $1.6 \mathrm{GHz}$ and should not affect the received power, thus making the antenna ideal for sensing changes in blood glucose levels.

The scope of this thesis includes the fabrication of the slot array antenna designed by Ho et al. with 3-dimensional electromagnetic simulations for power estimations shown in Chapter 3. Also included is the testing of the antenna on synthesized materials with varying dielectric constants relating to blood glucose concentrations common for 
diabetics in Chapter 4. Testing on human subjects from Cal Poly University is also included to gather data for noise variable analysis and the true feasibility of such a design. The concluding thoughts in Chapter 5 recap the likelihood of success for the antenna design, sources of error, and potential future work for the project. 


\section{Chapter 3: Design and Simulation}

Before beginning the design process, materials were first chosen to match the device requirements for durability. Sourcing the 4-port slot antenna with a single output signal generator was also determined with the design of a 4-way Wilkinson power divider. The antenna design process then followed multiple design-and-simulate iterations to determine the optimal strategy for glucose sensing. Design simulations were completed in Advanced Design System, a high-frequency schematic and layout analysis tool by Keysight Technologies. Additionally, the resulting design was simulated in a 3-D finite element modeler called EMPro for analyzing the antenna's performance through a model of the biceps brachii. The design was completed with a receiver to measure the radiated signal strength at the opposite end of the biceps brachii.

\subsection{Selection of Materials}

Commercial broadband antennas, military radar antennas, and microstrip circuits are all commonly designed and fabricated on a high frequency, copper-clad, laminate substrate called Duroid. Manufactured by Rogers Corporation, this substrate is a glass microfiber reinforced PTFE composite engineered for milling microstrips and striplines used in high frequency designs [34]. Its flexibility allows for easier cutting, shearing, and machining for high frequency circuits and usually has a consistently low energy dissipation factor across a wide frequency range. However, analysis of the physical and electrical parameters of Duroid determined that another substrate might be more applicable for the glucose sensor.

The main drawback of Duroid was its high flexibility, which could prove difficult for the setup of the glucose sensor. A more flexible substrate could potentially cause 
bending of the antenna source when placed at the designed distance of $1 \mathrm{~cm}$ away from the surface of the skin. Any bend in the source substrate would create inconsistencies between the measured values and the simulations, thus creating an unknown change in the accuracy of the sensor. This determination led to further research resulting in another option for the antenna source substrate.

A search through other materials offered by Rogers Corporation led to the discovery of another copper-clad substrate for high frequency design. This new substrate, called RO4350, was found to have a higher tensile modulus in both the x-direction and ydirection. The electrical properties of RO4350 also matched up to the superiority of Duroid with low dielectric loss and even surpassed Duroid's thermal coefficient of expansion [35]. The thermal coefficient of expansion determines how much a material will expand when introduced with energy in the form of heat. Therefore, a lower coefficient proves more valuable in a biomedical device application such as glucose sensing where material size should remain constant in a wide range of environmental or ambient temperatures. A board thickness also needed to be determined since RO4035 is offered in thicknesses ranging from $0.203 \mathrm{~mm}$ to $1.524 \mathrm{~mm}$. A thickness of $1.524 \mathrm{~mm}$ was ultimately chosen to match the design choice from Ho et. al [27]. To summarize, Table 3 shows a direct comparison of physical and electrical properties of interest between $1.5 \mathrm{~mm}$ thick Duroid and $1.524 \mathrm{~mm}$ thick RO4350 substrates from Rogers Corporation. 
Table 3: Substrate comparisons between FR-4/Duroid 5880 and RO4350 from Rogers Corporation.

\begin{tabular}{|c|c|c|}
\hline Property & Duroid 5880 & RO4350 \\
\hline \begin{tabular}{c} 
Dielectric Constant $\varepsilon_{\mathrm{r}}$ \\
\hline $\begin{array}{c}\text { Coefficient of Thermal Expansion in X-direction } \\
{\left[\mathrm{ppm} /{ }^{\circ} \mathrm{C}\right]}\end{array}$
\end{tabular} & 2.2 & 3.66 \\
\hline $\begin{array}{c}\text { Coefficient of Thermal Expansion in Y-direction } \\
{\left[\mathrm{ppm} /{ }^{\circ} \mathrm{C}\right]}\end{array}$ & 48 & 10 \\
\hline $\begin{array}{c}\text { Coefficient of Thermal Expansion in Z-direction } \\
{\left[\mathrm{ppm} /{ }^{\circ} \mathrm{C}\right]}\end{array}$ & 237 & 32 \\
\hline $\begin{array}{c}\text { Tensile Modulus in X-direction at } 23^{\circ} \mathrm{C}[\mathrm{kpsi}] \\
\text { Tensile Modulus in Y-direction at } 23^{\circ} \mathrm{C}[\mathrm{kpsi}]\end{array}$ & 156 & 2432 \\
\hline
\end{tabular}

In addition to selecting the substrate material, the type of coaxial cable used for the source connections was also determined. Standard copper $50 \Omega$ semi-rigid coaxial cables by Micro-Coax were selected due to their immediate availability on campus as well as their physical and electrical properties. This semi-rigid coaxial cable also has low attenuation and complete shielding for preventing signal loss and noise therefore providing great functional versatility [36]. The physical flexibility of the cable was additionally important since variations in the size of biceps brachii require movement of the source position and angle to appropriately fit each user, a necessity that highly affects the accuracy of the sensed glucose levels. The Micro-Coax semi-rigid cables provided these necessary electrical and physical parameters to uphold the legitimacy of the sensor design. Upon selection of these materials, the next step in preparing the source design was to design an excitation method for all four input ports on the slot array antenna.

\subsection{Preliminary Source Excitation Designs}

Bench-top high-frequency signal generators equipped in Cal Poly labs produce a single output with frequency modulation, amplitude modulation, and other variable 
parameters for generated signals up to $1 \mathrm{GHz}$. Interfacing a 4-port slot array antenna at 1.6 GHz with a single-output signal generator therefore required a frequency mixer and a 4-way power splitter to source all four inputs.

\subsubsection{A 1.6 GHz Signal Generator}

In electronics, frequency mixers are often used to produce a signal at a desired sum or difference of two inputs, a signal and a local oscillator. However, the mixer produces potentially unwanted harmonics at nearby frequencies of the outputs that could create noise in the system and detract from the receiver's accuracy. These additional harmonics are typically removed with a bandpass filter centered at the desired frequency.

For the ease of design efforts, a fellow graduate student designed the frequency mixer needed to produce $1.6 \mathrm{GHz}$ [37]. The frequency mixer was designed with the ADE 42MH integrated circuit to combine a $1 \mathrm{GHz}$ input signal and a $600 \mathrm{MHz}$ local oscillator signal to produce two outputs - one at $1.6 \mathrm{GHz}$ and another at $400 \mathrm{MHz}$. A bandpass filter was then designed and simulated in ADS to remove unwanted signals at $400 \mathrm{MHz}$ and additional harmonics while keeping the desired signal at $1.6 \mathrm{GHz}$.

A fifth-order bandpass filter design was implemented for a frequency of this magnitude. The design incorporated five microstrips, each a quarter-wavelength long, to act as an open circuit for the desired frequency and a short circuit for unwanted frequencies. Simulations of the bandpass filter showed a $-1.7 \mathrm{~dB}$ loss at the pass band while rejecting other signals by $-62 \mathrm{~dB}$. This signal rejection also included the $400 \mathrm{MHz}$ output of the frequency mixer, as desired. Figure 14 shows the schematic of the bandpass filter designed by fellow graduate student Dawei Zhang. 


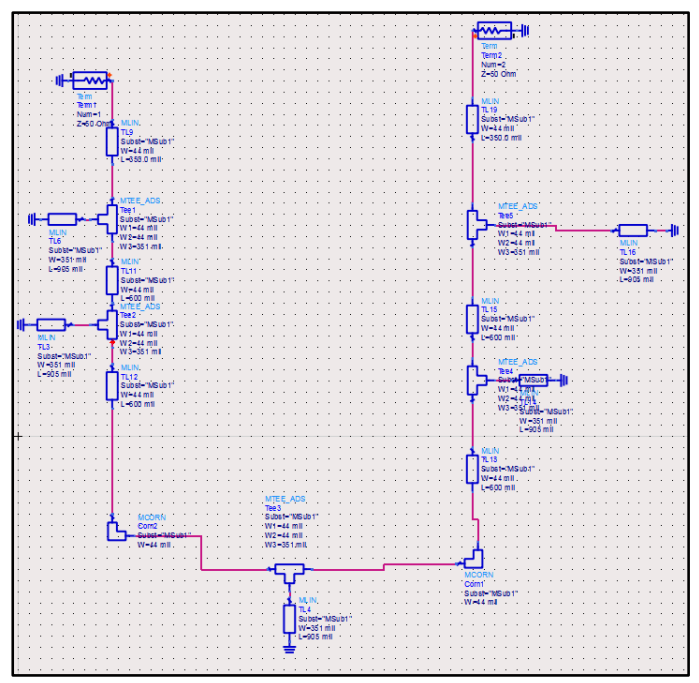

Figure 14: Bandpass filter designed for extracting unwanted output signals from the frequency mixer [37].

\subsubsection{Four-Output Wilkinson Power Divider}

The original two-output Wilkinson power divider uses two quarter-wavelength microstrips of $1.414 \mathrm{Z}_{0} \Omega$ to divide a single input into two equal outputs. The design also incorporates a $2 Z_{0} \Omega$ resistor placed across the outputs to dissipate reflected power from these ports. When all ports are impedance matched, each output contains half the power of the input, resulting in $-3 \mathrm{~dB}$ loss with no phase shift [38]. To build off the original design and create a 4-way power split, one divider was designed with its two outputs each connected to an additional 2-way divider. The branched dividers were designed to produce four separate outputs with a target loss of $-6 \mathrm{~dB}$ at equal phase shift.

An initial design for the 2-way divider was set up in ADS schematic and simulated with a substrate matching RO4350 parameters. Termination ports of $50 \Omega$ were selected to match the port impedance of the signal generator and semi-rigid coaxial cables. The ADS tool called "LineCalc" was used to determine the lengths and widths necessary for creating $1.414 \mathrm{Z}_{0} \Omega$ of impedance with a quarter-wavelength phase shift, 
and the resulting lengths and widths were $28.38 \mathrm{~mm}$ and $1.75 \mathrm{~mm}$, respectively. Instead of using a physical resistor of $2 \mathrm{Z}_{0} \Omega$ across the output ports, a microstrip with identical impedance was calculated with LineCalc and placed at the output ports. Additional microstrip lines were placed to separate the quarter-wavelength lines at a distance matching the $2 Z_{0} \Omega$ resistor's length. Figure 15 shows the schematic view of the 2 -way Wilkinson power divider.

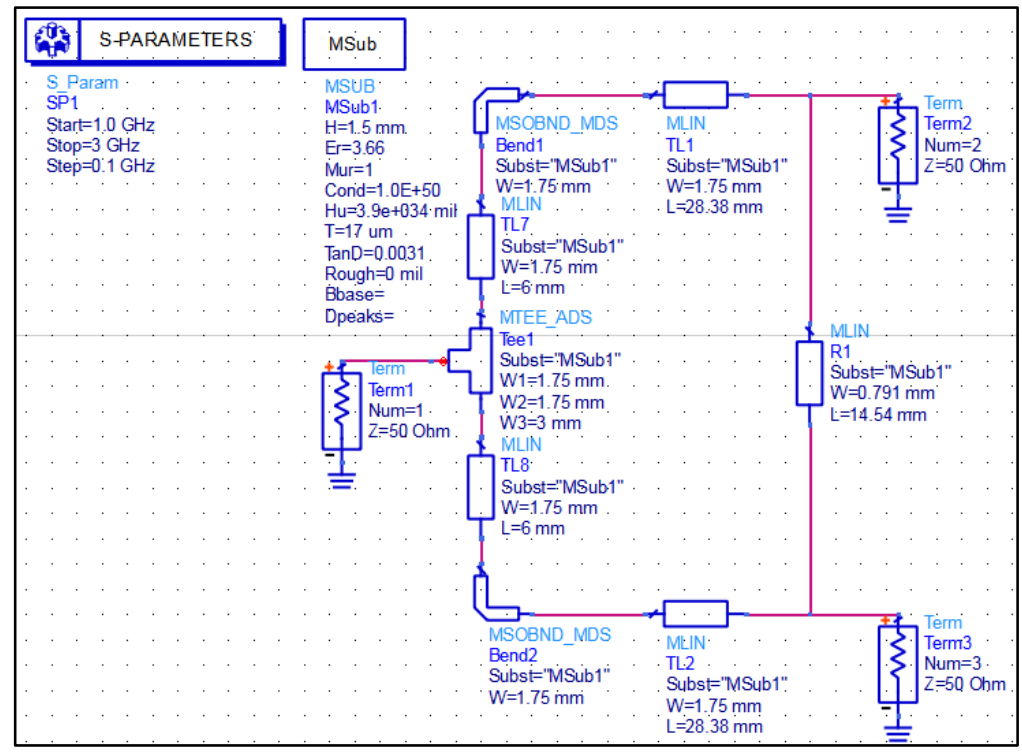

Figure 15: The 2-way Wilkinson power divider schematic designed in ADS. The microstrip lengths and widths were calculated to match the desired impedance at 1.6

\section{$\mathrm{GHz}$.}

Upon completion of the schematic, the power splitter was simulated across a frequency range of $1 \mathrm{GHz}$ to $3 \mathrm{GHz}$. The resulting S-parameters showed the desired -3 $\mathrm{dB}$ loss at the two output ports, as shown in Figure 16. A phase shift of $-123^{\circ}$ was also calculated by ADS, and these were due to the added microstrip lines for resistive purposes. However, the value was neglected since both outputs were phase shifted by the 
same amount, and any specific phase shifting could be controlled after passing through the divider board if necessary.
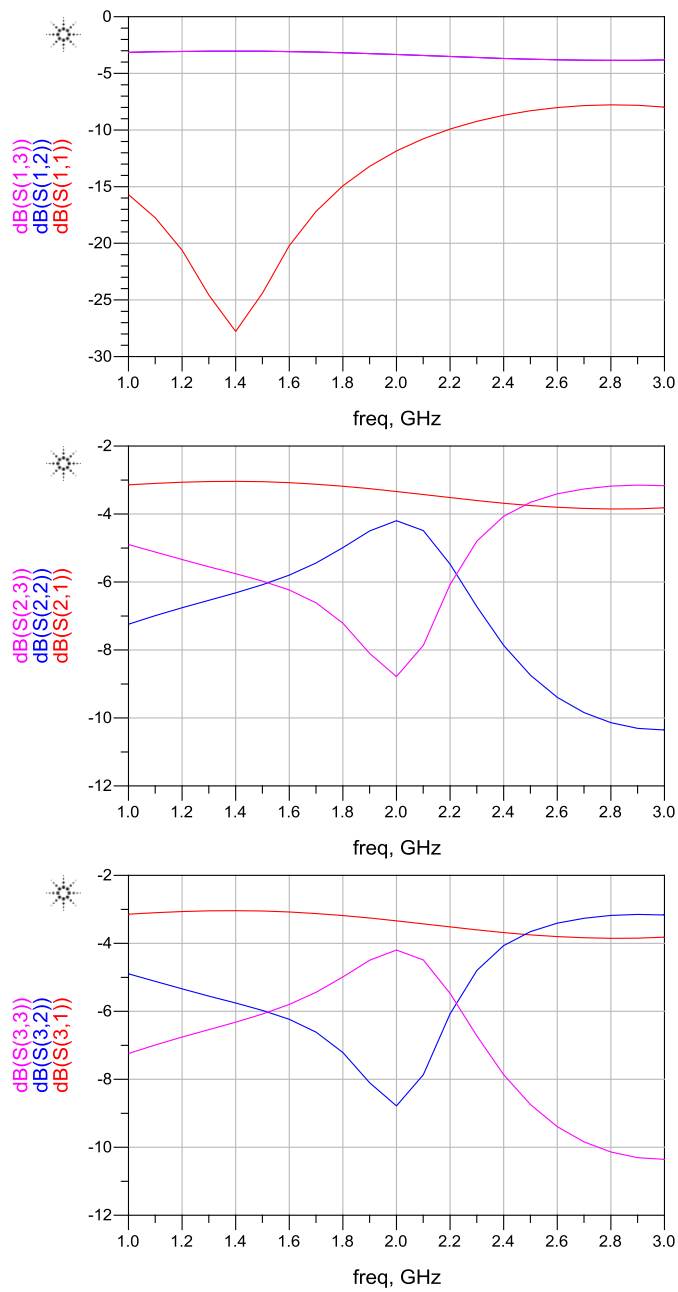

Figure 16: The S-parameters resulting from the simulation of a 2-way Wilkinson power divider proves the anticipated $3 \mathrm{~dB}$ loss at the two outputs at $1.6 \mathrm{GHz}$. After verifying the 2-way Wilkinson power divider, two identical dividers were connected to the outputs of the original splitter to create a 4-way divider. The resulting schematic is seen in Figure 17, and the layout generated from the schematic is shown in Figure 18. Upon simulation of the layout, microstrip lengths at the outputs were edited to bring the ports as close to $-6 \mathrm{~dB}$ loss as possible. Table 4 provides the magnitudes and 
phases of the resulting S-parameters at the four output ports. After completing the design of the 4-way power splitter to the slot array antenna, all design parameters were sufficiently completed to begin designing the slot array source.

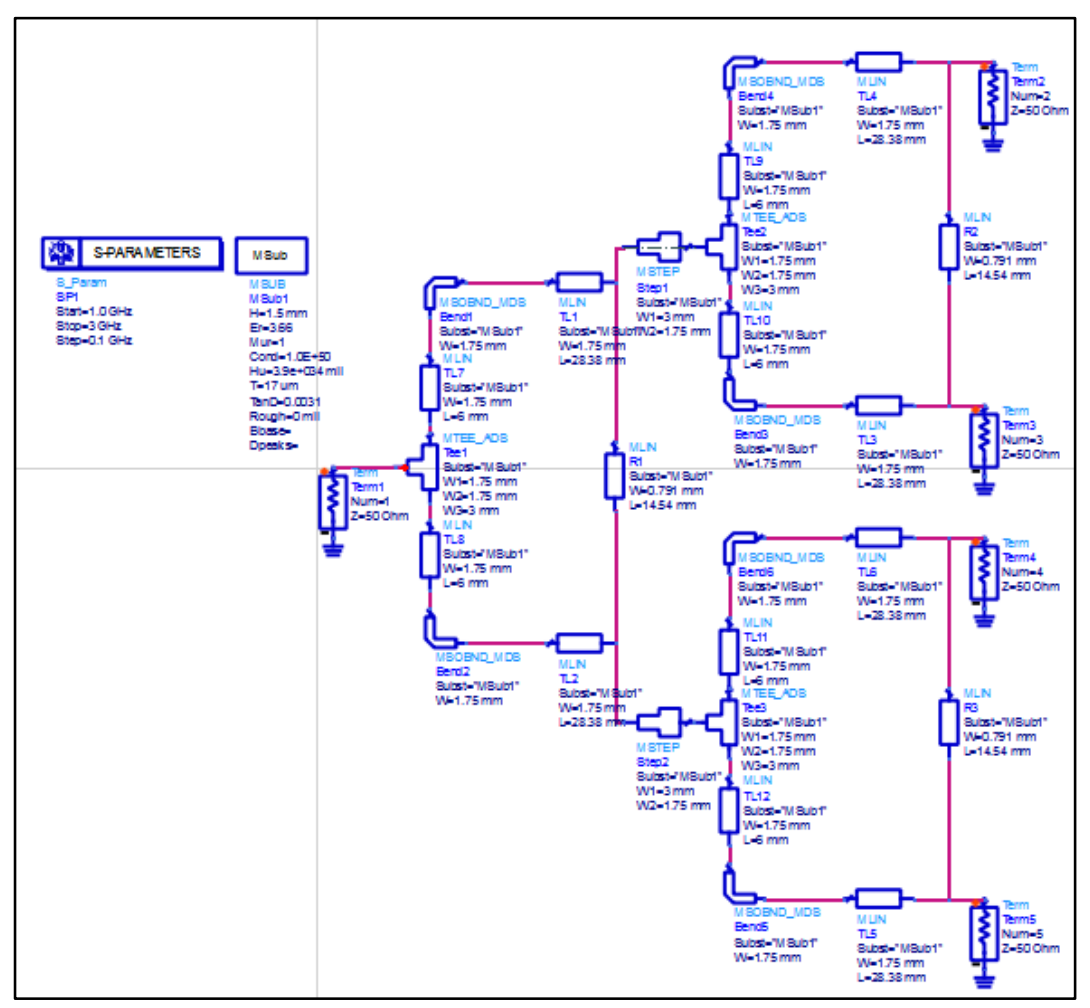

Figure 17: ADS schematic of the 4-way Wilkinson power divider derived from the 2-way splitter.

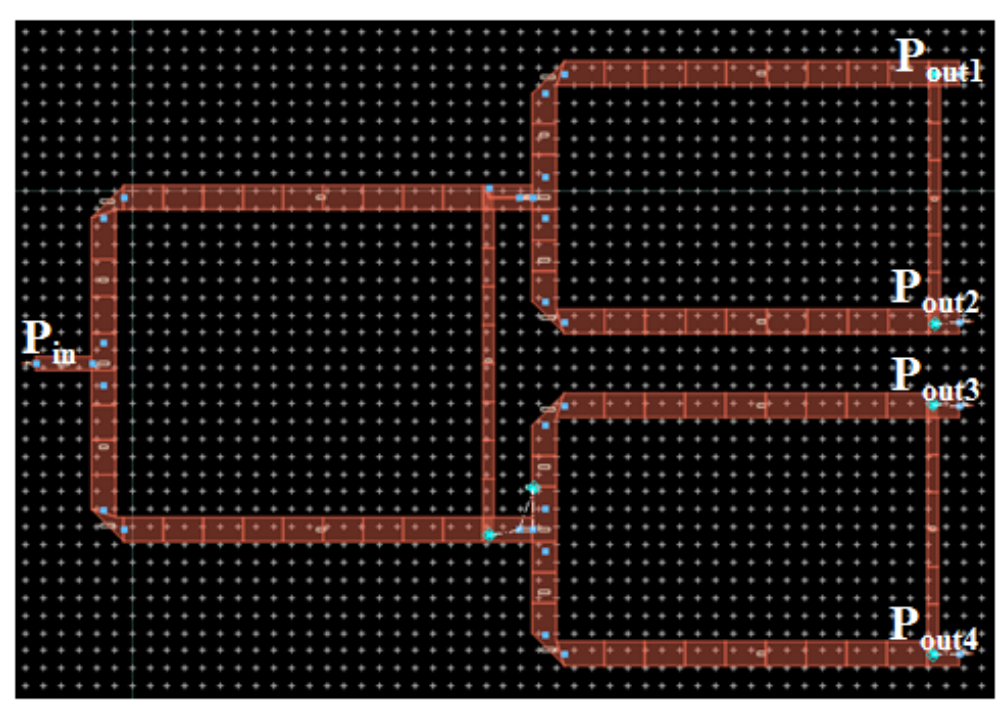


Figure 18: Corresponding layout of 4-way Wilkinson power divider with the single input port (Port 1) at the left and four separate outputs (Ports 2-5) on the right. The vertical microstrips are $100 \Omega$ resistors to dissipate reflections at the outputs.

Table 4: Simulated S-parameters for the outputs of the 4-way Wilkinson power divider show near ideal values. Due to reciprocity, the output ports have the same power when looking from the source to the output.

\begin{tabular}{|c|c|c|}
\hline Parameter & Magnitude [dB] & Phase [degrees] \\
\hline $\mathrm{S}_{21}=\mathrm{S}_{12}$ & -6.128 & 77.7 \\
\hline $\mathrm{S}_{31}=\mathrm{S}_{13}$ & -6.178 & 77.1 \\
\hline $\mathrm{S}_{41}=\mathrm{S}_{14}$ & -6.182 & 77.1 \\
\hline $\mathrm{S}_{51}=\mathrm{S}_{15}$ & -6.124 & 77.7 \\
\hline
\end{tabular}

\subsection{Single-Slot Antenna Design}

Based on the information provided by Kim et al. [30], an approximated physical realization of the source was determined. Specific information included in [30] revealed that the radius of the curved slot shape was about $3 \mathrm{~cm}$. However, the exact dimensions of the remaining circular shapes shown in [27] were not provided and therefore estimated based on visual inspection.

\subsubsection{Increasing Radiation Efficiency}

The best design approach for the overall slot antenna was to optimize the power transfer efficiency of a single slot design from the full array. Based on this logic, a generic layout was created in ADS to mimic the three shapes specified by Ho et al. as seen in Figure 19. Multiple shape dimensions were then simulated and tested to optimize the power transfer efficiency of the single slot. 


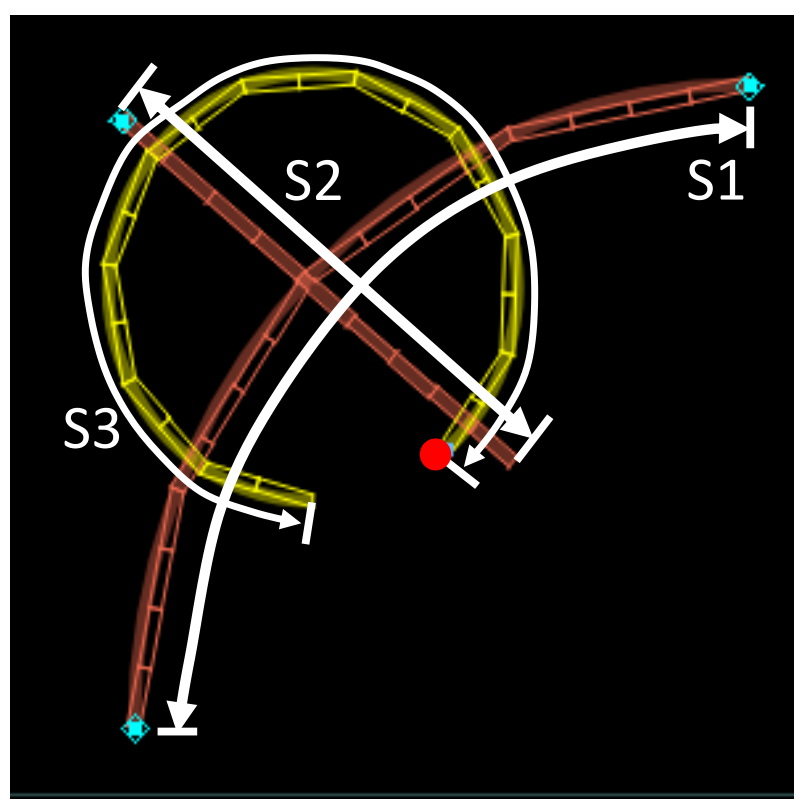

Figure 19: The single slot antenna design was configured in six different ways based on size parameters of shapes S1, S2, and S3. The yellow semi-circle represents the top microstrip layer, and the red arc and line represent the bottom slot layer.

The single slot antenna pattern was designed to match a termination load of $50 \Omega$ at the input and output ports. The measurement ports were placed at the red circle indicator in Figure 19 with the positive port connected to the top microstrip layer and negative port connected to the bottom slot layer of a RO4035 substrate. Table 5 lists the six different designs tested and their corresponding shape dimensions.

Table 5: Various sizes for shapes S1, S2, and S3 were tested to find the maximum power transfer efficiency in free space.

\begin{tabular}{|c|c|c|c|c|c|c|c|c|}
\hline \multirow{2}{*}{$\begin{array}{c}\text { Design } \\
\text { Name }\end{array}$} & \multicolumn{3}{|c|}{ Shape 1 (S1) } & \multicolumn{2}{c|}{ Shape 2 (S2) } & \multicolumn{3}{c|}{ Shape 3 (S3) } \\
\cline { 2 - 9 } & $\begin{array}{c}\text { Radius } \\
{[\mathbf{c m}]}\end{array}$ & $\begin{array}{c}\text { Width } \\
{[\mathbf{m m}]}\end{array}$ & $\begin{array}{c}\text { Angle } \\
{[\text { degrees] }}\end{array}$ & $\begin{array}{c}\text { Length } \\
{[\mathbf{c m}]}\end{array}$ & $\begin{array}{c}\text { Width } \\
{[\mathbf{m m}]}\end{array}$ & $\begin{array}{c}\text { Radius } \\
{[\mathbf{c m}]}\end{array}$ & $\begin{array}{c}\text { Width } \\
{[\mathbf{m m}]}\end{array}$ & $\begin{array}{c}\text { Angle } \\
{[\text { degrees] }}\end{array}$ \\
\hline L1 & 3 & 0.635 & 90 & 2.5 & 0.635 & 1 & 0.635 & 320 \\
\hline L2 & 3 & 1 & 80 & 3 & 1 & 0.9 & 0.635 & 310 \\
\hline L3 & 3 & 1 & 80 & 3 & 1 & 0.9 & 1 & 310 \\
\hline L4 & 3 & 1 & 80 & 3 & 1 & 0.9 & 2 & 310 \\
\hline
\end{tabular}




\begin{tabular}{|l|l|l|l|l|l|l|l|l|}
\hline L5 & 3 & 1 & 80 & 3 & 2 & 0.9 & 1 & 310 \\
\hline L6 & 3 & 3 & 80 & 3 & 3 & 0.9 & 2 & 310 \\
\hline
\end{tabular}

With each simulation, the input port was excited with a sinusoidal wave of $1 \mathrm{~V}$ amplitude and no phase shift. Simulation results from ADS produced the total power radiated in free space and the input power of the antenna, both in Watts. Total radiated power was calculated from the free space far-field electrical and magnetic components and integrated over half of the open space [39].

$$
P_{\text {rad }}=\int_{\Omega} U(\theta, \varphi) d \Omega=\frac{1}{2} \int_{\Omega} E_{f f} \times H_{f f}^{*} d \Omega
$$

Input power was calculated by computing the difference between the input and reflected power in Watts delivered to the antenna. The maximum power efficiency was then calculated by determining the ratio between the radiated power and input power computed by ADS. The resulting values are shown in Table 6.

Table 6: Based on the input power and radiated power as calculated by ADS, the radiation efficiency was determined to choose the optimal slot antenna design.

\begin{tabular}{|c|c|c|c|}
\hline Design & $\begin{array}{c}\text { Input Power } \\
{[\mathbf{m W}]}\end{array}$ & $\begin{array}{c}\text { Radiated Power } \\
{[\mathbf{m W}]}\end{array}$ & $\begin{array}{c}\text { Radiation Efficiency } \\
{[\%]}\end{array}$ \\
\hline L1 & 1.26502 & 1.021800 & 80.773 \\
\hline L2 & 0.196343 & 0.127395 & 64.884 \\
\hline L3 & 0.342983 & 0.233825 & 68.174 \\
\hline L4 & 0.971336 & 0.763782 & 78.632 \\
\hline L5 & 2.14588 & 1.671950 & 77.914 \\
\hline L6 & 1.02228 & 0.841807 & 82.346 \\
\hline
\end{tabular}

Determining the optimal design depended on a combination of the radiation efficiency parameter along with the calculated input power. The first and most significant figure of merit was the radiation efficiency. Radiation efficiencies greater 
than $75 \%$ were assumed to be optimal and thus narrowed down the design choices to L1, L4, L5, and L6. The second figure of merit for consideration was the antenna's calculated input power. A large input power meant less reflected power from the input ports of the antenna, which allowed for greater power delivered to the source.

Considering this figure of merit, L5 would have been the best choice for implementation. However, all remaining design choices were so similar in radiation efficiency that L1, L4, L5, and L6 were all considered in the next design step.

Figure 20 presents a visual comparison of the simulated designs. This graph shows the direct correlation between input power and radiated power; but independently, these parameters do not directly affect the radiation efficiency.

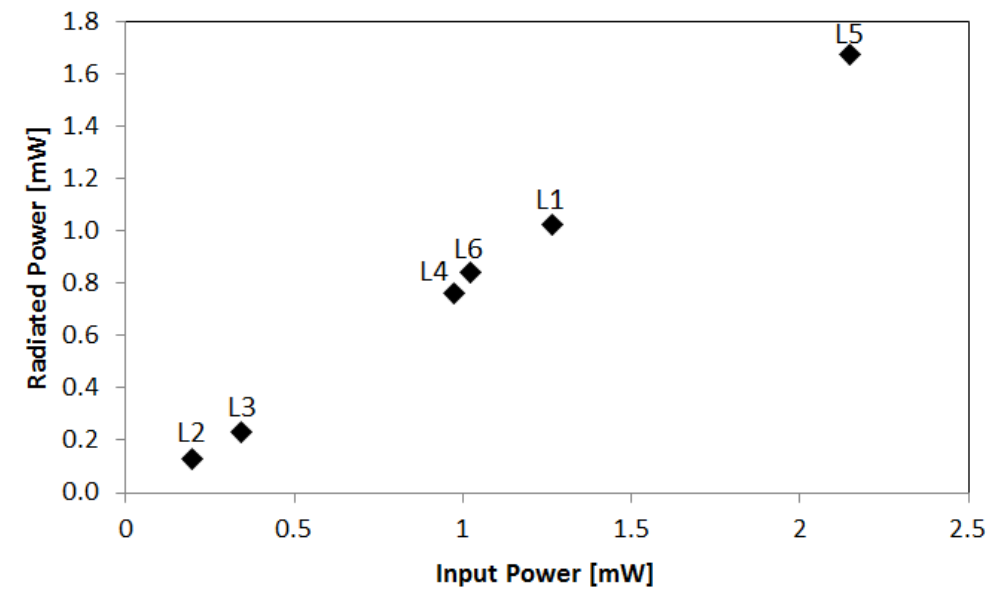

Figure 20: A graphical representation of the radiated power based on input power for all six designs shows the variation in each of the two parameters.

\subsubsection{Impedance Matching for Single-Slot Antenna Design}

The next design step was to determine the best slot layout with optimal scattering parameters at $1.6 \mathrm{GHz}$. When designing an antenna at high frequencies, the scattering parameters of the design determine the overall performance of the antenna because they characterize the amount of voltage received from a source in comparison to the amount of 
voltage actually used by the antenna. Scattering parameters are especially necessary at frequencies in the gigahertz range since any connecting paths to and from the design can cause signal reflections. These signal reflections create standing waves on the connecting paths and degrade the actual amount of voltage at the microstrip [40]. Therefore, radiated power efficiency and overall antenna performance rely on optimizing scattering parameters by minimizing signal reflections.

Simulating the $S_{11}$ scattering parameter, or voltage received at the first port in relation to voltage used from the same port, was the best way to optimize the remaining designs. Ideally, this parameter falls between $-10 \mathrm{~dB}$ and $-15 \mathrm{~dB}$ as an indication of less signal reflection from the microstrip layer and can vary based on the placement of the input port on the microstrip. Designing port locations to create the lowest $S_{11}$ measurements is called impedance matching.

$\mathrm{S}_{11}$ measurements were taken on the single slot designs of L1, L4, L5, and L6 with varying port locations to find the position with least reflections. Figure 21 indicates the nine locations of which $S_{11}$ parameters were measured for impedance matching each design. 


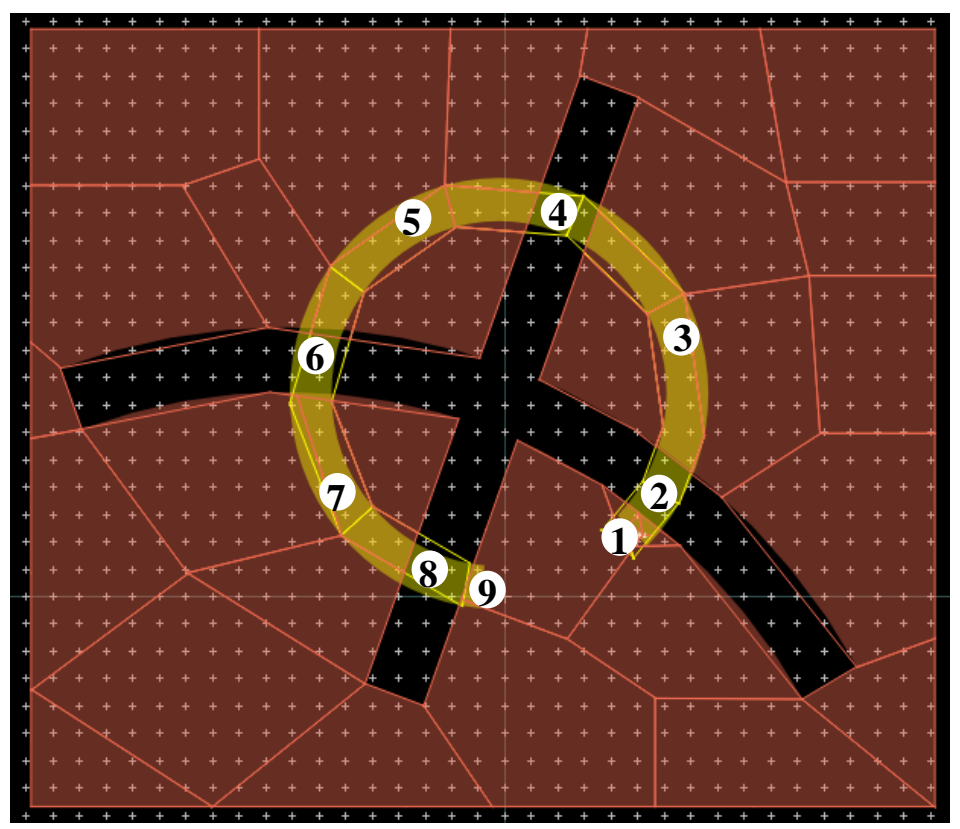

Figure 21: To find the optimal point of excitation for the single slot antenna design, $\mathrm{S}_{11}$ parameters were acquired at the identified nine points around the microstrip curve.

The first position for the input and output port was at the end of the circular microstrip labeled " 1 " in Figure 21. Nine measurements were taken around the microstrip until reaching the opposite end. Figure 22 graphs the resulting $S_{11}$ parameters at each position for all four designs. Design L4 was chosen since the lowest $\mathrm{S}_{11}$ parameter measured far below the necessary threshold at about $-20 \mathrm{~dB}$. 


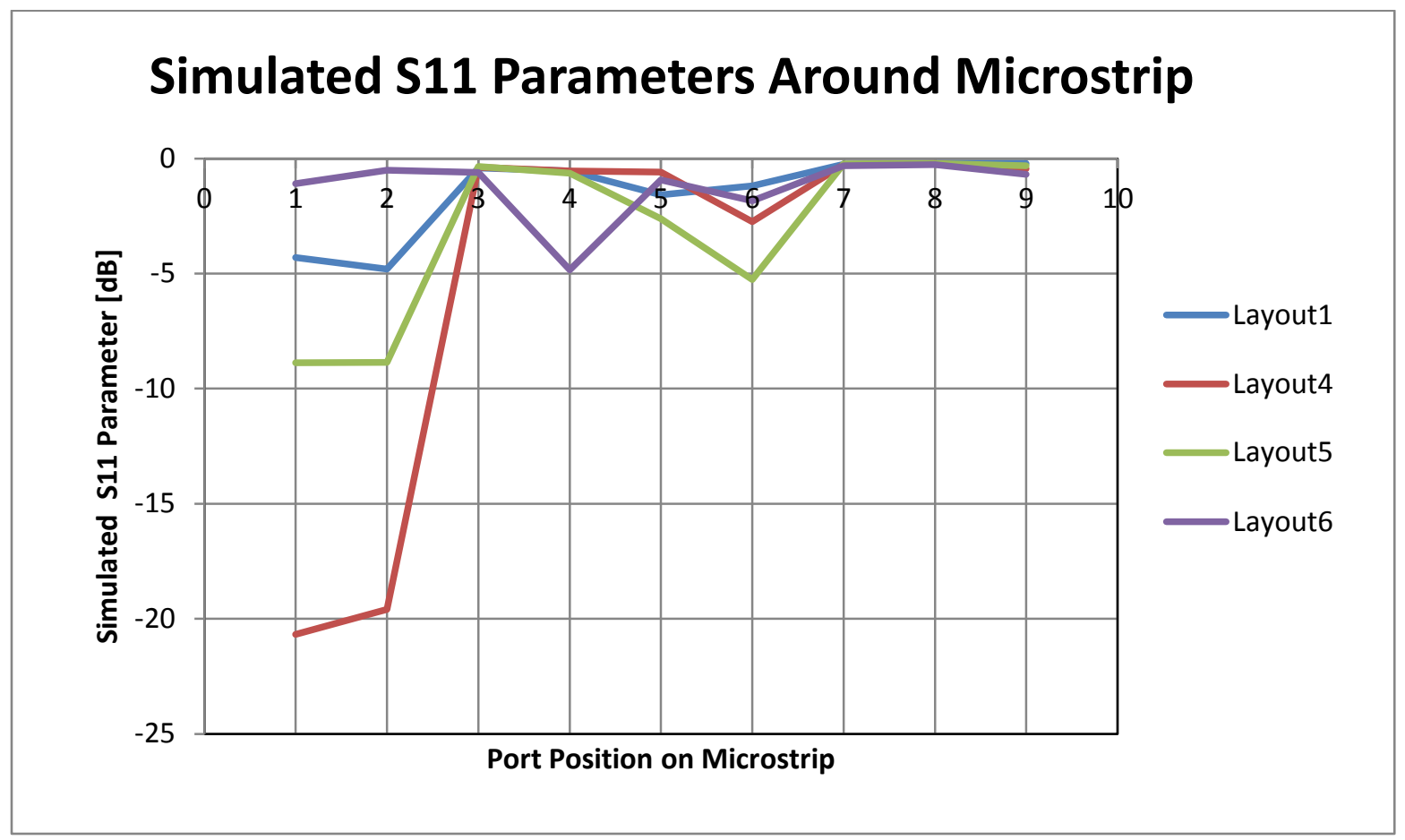

Figure 22: The simulated $S_{11}$ parameters of all four layout designs (L1, L4, L5, and L6) determined the best input placement for minimal signal reflection.

The dimensions shown in Table 5 were used to create the physical layout of L4, as seen in Figure 23. The ADS simulation of the single slot design with a $1 \mathrm{~V}$ sinusoidal input at $1.6 \mathrm{GHz}$ also calculated the maximum current density of the design. The maximum current density reached $11.91 \mathrm{~A} / \mathrm{m}$ and is indicated by the warm red color at the input port on the microstrip layer. Cool blue colors indicate little to no current density on the board. The simulation also showed that current density increased near the ends of the curved slots, which indicated radiation on both sides of the plane of the antenna. 


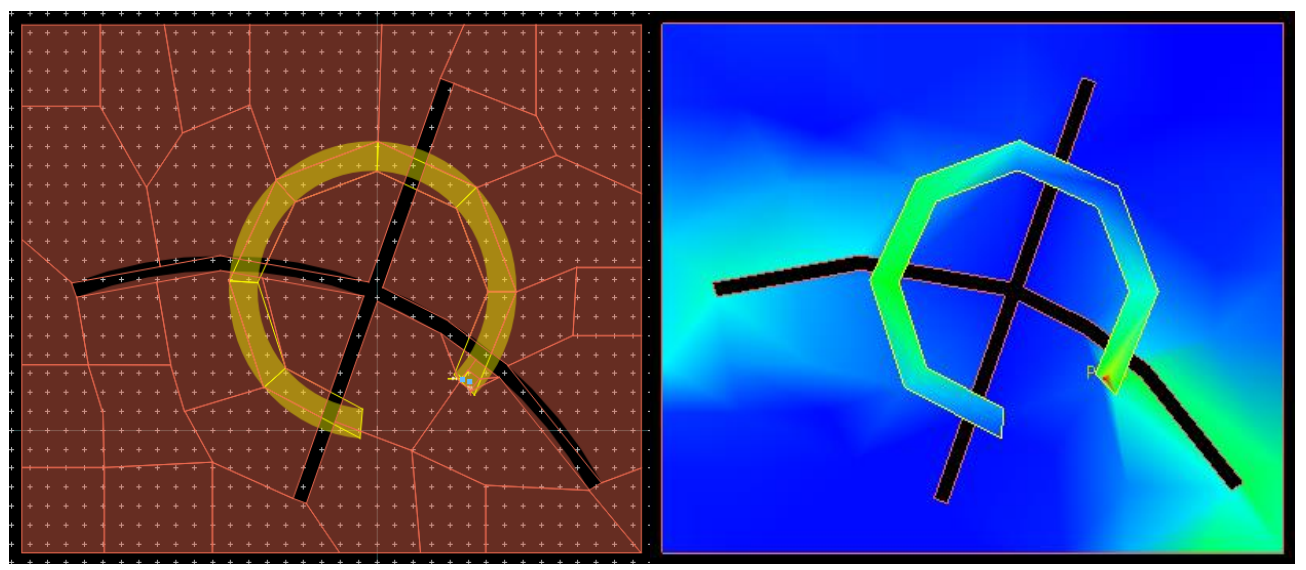

Figure 23: Physical layout of design L4 (left) and its current density (right). The maximum current density achieved was $11.91 \mathrm{~A} / \mathrm{m}$.

A 3-D far field visualization of the antenna pattern was also realized by ADS.

Figure 24 shows the produced antenna pattern and what appears to be symmetry across the plane of the antenna. To verify this estimation, plots of the polar magnitudes of gain, radiation, and magnetic fields were produced by ADS. Figure 25 shows the polar magnitudes of these parameters at the plane indicated by the green line in Figure 24 . 


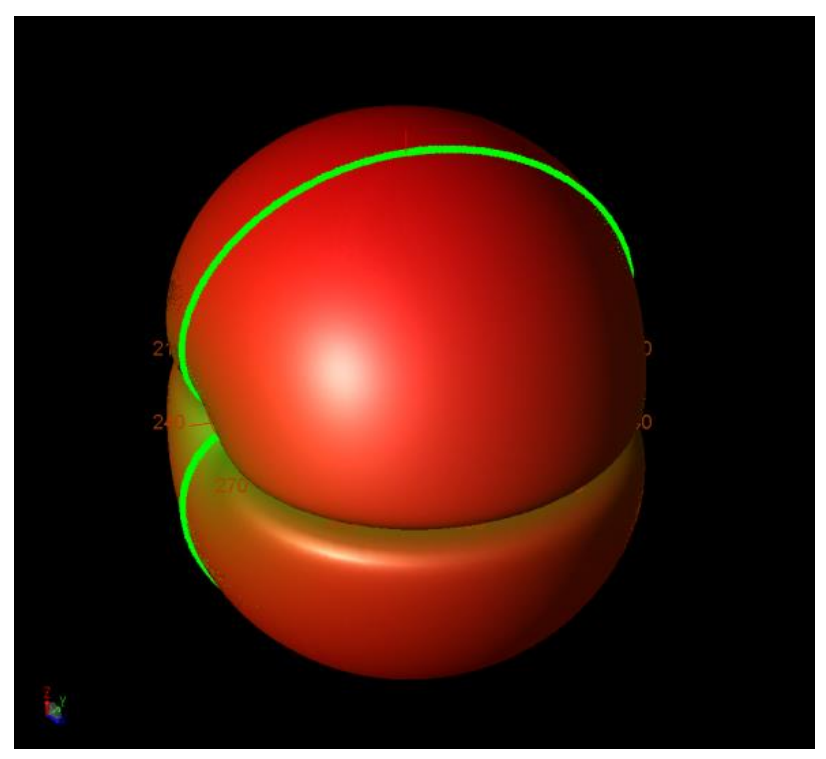

Figure 24: The antenna pattern of the single slot design shows a symmetrical pattern about the z-axis. Far field measurements were recorded for the cut plane at a polar angle of 60 degrees indicated by the green line.
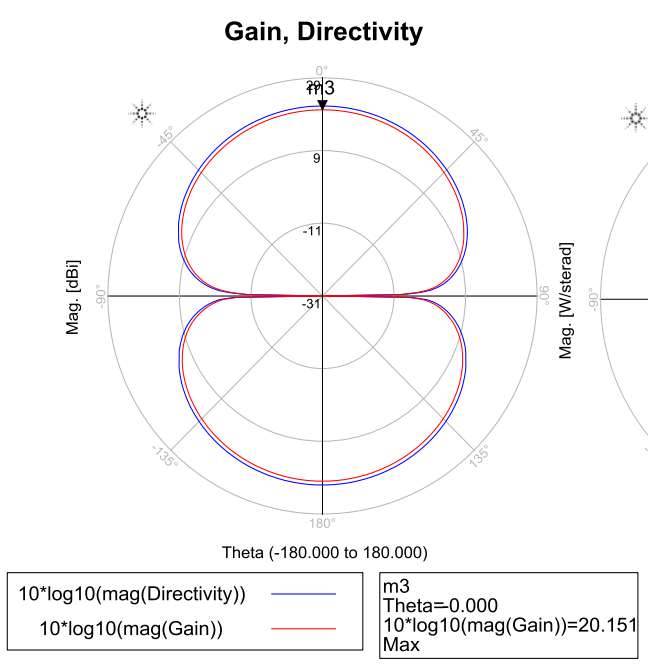
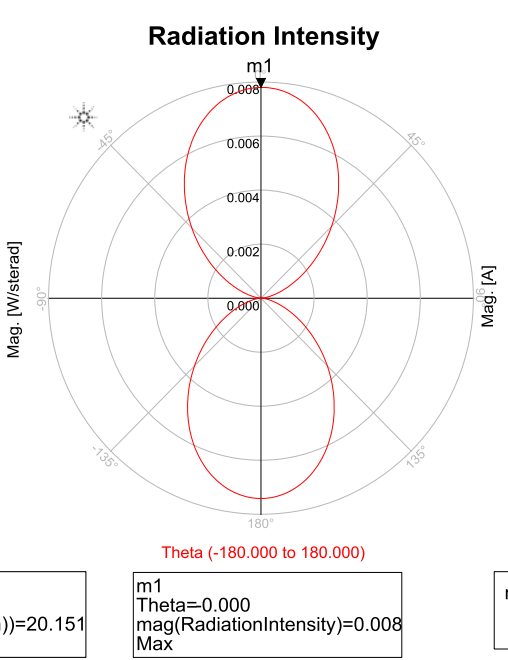
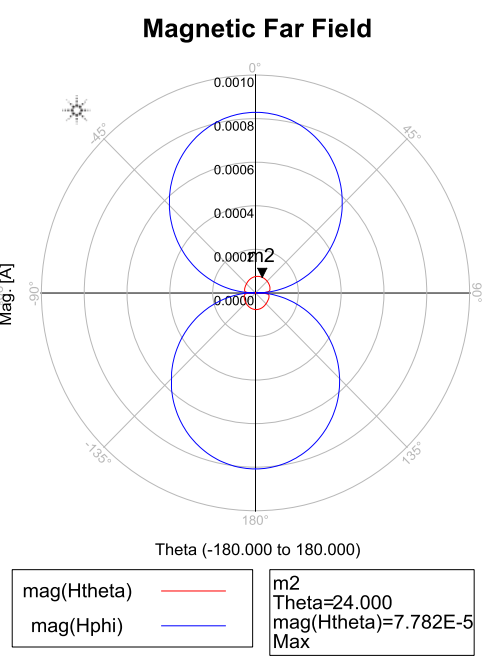

Figure 25: At a far field plane of 60 degrees, the maximum gain of the antenna was near

$20 \mathrm{dBi}$. The radiation intensity of the single slot antenna was $0.008 \mathrm{~W} / \mathrm{sterad}$, and the maximum magnetic far field was $-7.78 \mathrm{E}-5 \mathrm{~A} / \mathrm{m}$.

The far field polar plots revealed that the gain and directivity of the single slot layout were indeed symmetrical across the plane of the antenna, as were radiation and the 
magnetic far field. It is important to take notice of the maximum gain achieved in free space by the single slot antenna, which was calculated to be $20.151 \mathrm{dBi}$ by ADS. The maximum radiation intensity was also calculated to be $0.008 \mathrm{~W} /$ sterad. With the single slot simulations complete, the full slot array simulation was necessary to further characterize the source antenna.

\subsection{Slot Array Antenna Characterization}

The slot array antenna was characterized in free space using the ADS Microwave Momentum simulator with the same simulation setup as the single slot. Figure 26 shows the physical layout of the entire slot array antenna and the simulator's 3-D representation of the design, and Figure 27 shows the simulated current density with all ports equally sourced. Upon simulation, the S-parameters for all four input ports were calculated with the magnitude in decibels and phase in degrees as shown in Table 7.

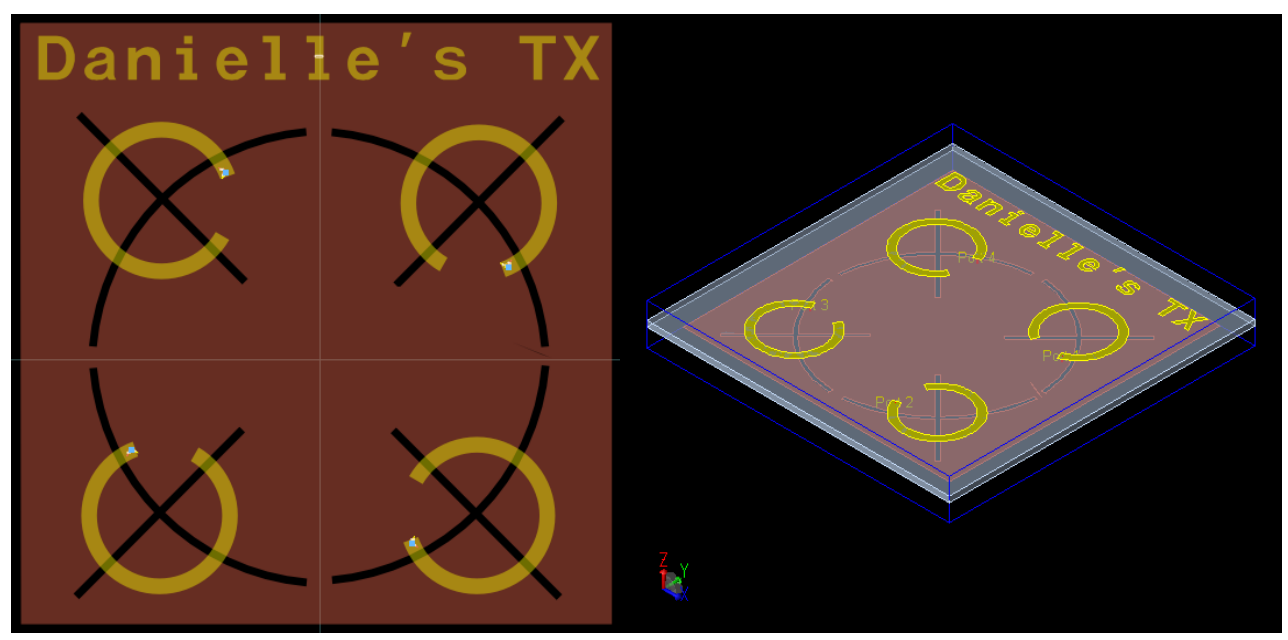

Figure 26: ADS layout design and 3-D representation of the full slot array antenna. The yellow shapes indicate the microstrip layer, and the dark red indicate the ground plane with slot shapes removed. 


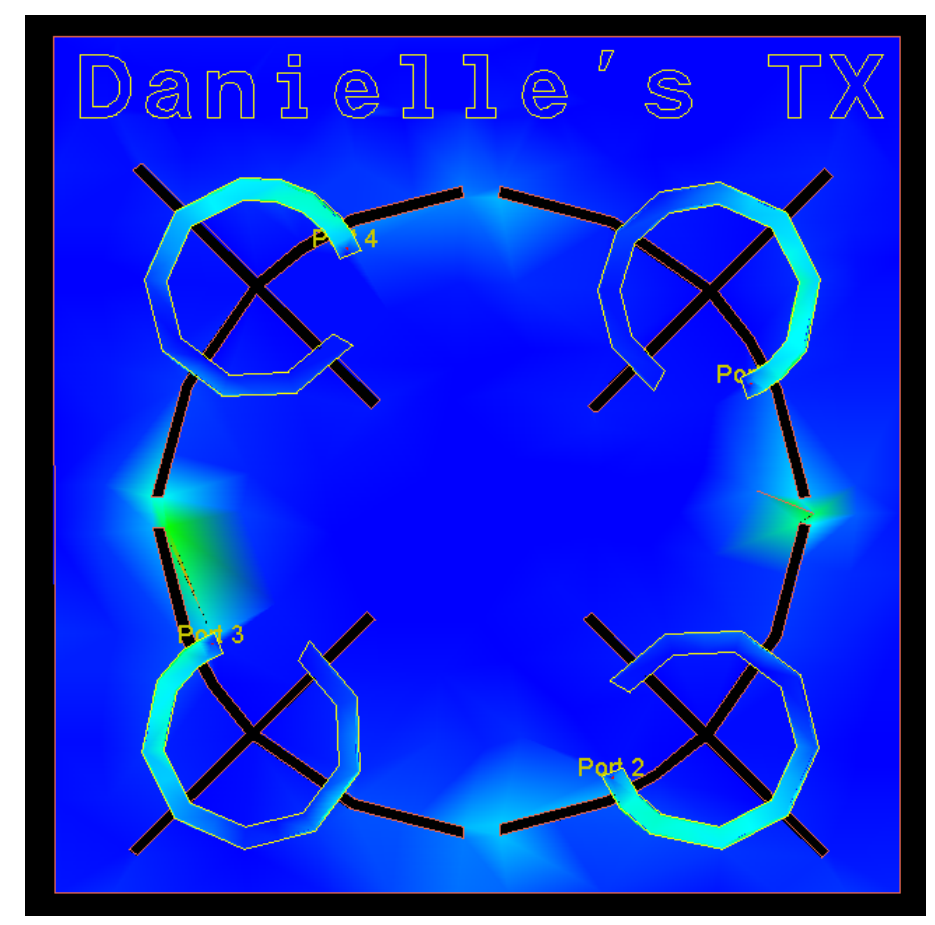

Figure 27: ADS simulation of the slot array achieved a current density of $17.77 \mathrm{~A} / \mathrm{m}$.

Table 7: Simulated S-parameters for the slot array antenna in free space.

\begin{tabular}{|c|c|c|}
\hline \multicolumn{3}{|c|}{ S-Parameters } \\
\hline Parameter & Magnitude [dB] & Phase [deg] \\
\hline $\mathrm{S}_{11}$ & -2.395 & 4.236 \\
\hline $\mathrm{S}_{12}$ & -11.437 & 78.666 \\
\hline $\mathrm{S}_{13}$ & -18.562 & 51.001 \\
\hline $\mathrm{S}_{14}$ & -13.073 & 87.4 \\
\hline $\mathrm{S}_{21}$ & -11.437 & 78.666 \\
\hline $\mathrm{S}_{22}$ & -2.829 & 11.898 \\
\hline $\mathrm{S}_{23}$ & -15.041 & 107.2 \\
\hline $\mathrm{S}_{24}$ & -21.412 & 50.081 \\
\hline $\mathrm{S}_{31}$ & -18.562 & 51.001 \\
\hline $\mathrm{S}_{32}$ & -14.517 & 107.2 \\
\hline $\mathrm{S}_{33}$ & -2.817 & 4.929 \\
\hline $\mathrm{S}_{34}$ & -15.972 & 116.6 \\
\hline $\mathrm{S}_{41}$ & -13.073 & 87.4 \\
\hline $\mathrm{S}_{42}$ & -21.412 & 50.081 \\
\hline $\mathrm{S}_{43}$ & -15.972 & 116.6 \\
\hline $\mathrm{S}_{44}$ & -1.993 & 1.271 \\
\hline
\end{tabular}


Since the antenna was simulated in free space, the large S-parameters measured by ADS were understood as a general characterization of the antenna in air. It was noted that these results were not typically desired of an antenna and were merely used to define the antenna's performance in free space without regards to the intended propagation through biological mediums.

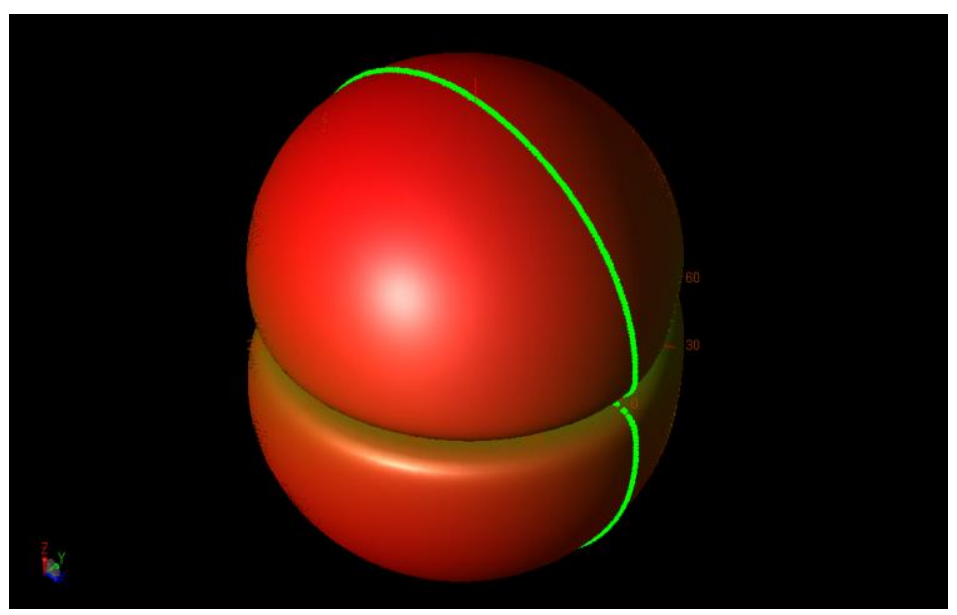

Figure 28: The antenna pattern of the full slot array in free space shows what appears to be a symmetrical radiation pattern above and below the plane of the antenna.

At a far field cut of zero polar angle, all fields radiated both above and below the plane of the antenna, as seen in Figure 28. The fields propagated at nearly a symmetrical pattern, yet radiation intensity was greatest above the plane of the antenna on the microstrip side. Despite the stronger radiation on the microstrip side of the antenna, the final design positioned the antenna to radiate from its ground plane into biological matter. This design choice was decided by [27] since its current density at the boundary between air and the body created the necessary pattern for optimal power transfer efficiency.

Figure 29 shows the resulting far field patterns described with the maximum gain near $20.25 \mathrm{dBi}$ and maximum radiation intensity of $0.007 \mathrm{~W} /$ sterad. Results also showed that the magnetic far field was nearly a magnitude greater than the single slot simulation 
at about $3.4 \mathrm{~A} / \mathrm{m}$. The radiation intensity pattern also showed the directivity of the antenna and the asymmetrical fields it produced when all inputs were of equal weight and phase.
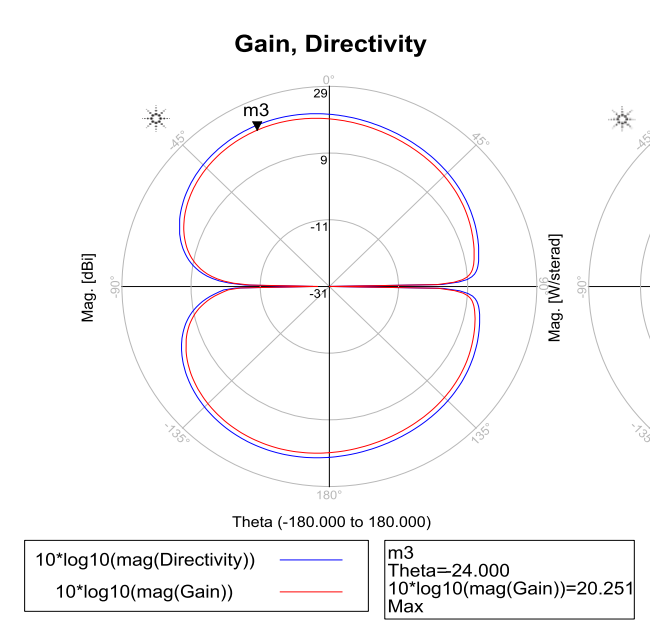
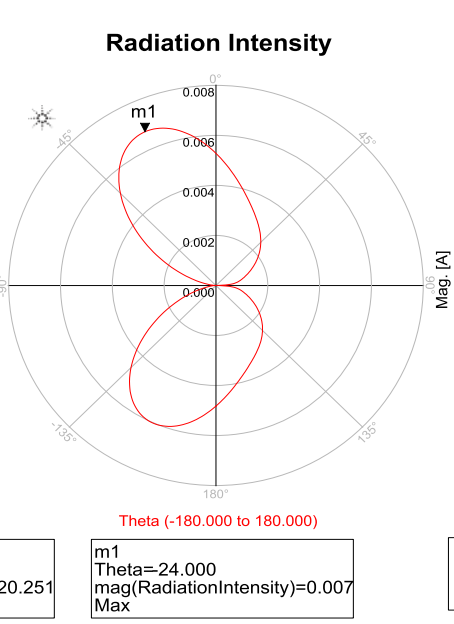

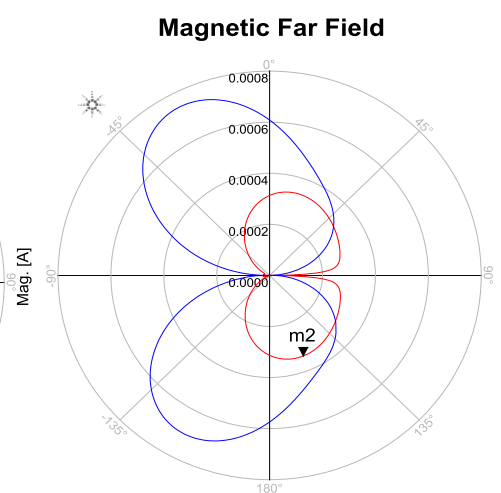

Theta $(-180.000$ to 180.000$)$

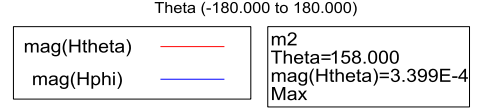

Figure 29: Gain, radiation, and magnetic far field patterns from a far field cut at a zero degree polar angle.

\subsubsection{Determining the Directionality of the Slot Array Antenna}

With the final slot array layout completed, the next design step was to optimize the controllability of the output field direction. Based on the work by [27], it was understood that an output direction in reference to the plane of the antenna could be achieved by changing the phase of the four input signals. This information allowed for an analysis of simulations with varied input settings to control the direction of the output fields. Directional controllability was necessary to minimize field contact with bone for reasons including radiation safety and signal loss incurred from the bone material. Any interference from bone would ultimately cause unknown changes in the received signal and thus degrade the accuracy of the sensor. 
To determine the field waves through all layers of propagation, a 3-D model was set up and simulated in both ADS and EMPro, a companion 3-D RF modeling software by Keysight Technologies. The compatibility between the two software suites allowed for antenna design and layout in ADS, including port placements and substrate definition, completed by 3-D finite element field measurements in EMPro. The antenna was first simulated on a $4 \mathrm{~cm}$ layer of blood separated by a $1 \mathrm{~cm}$ air gap. The modeled blood was set as an electrically isotropic material with magnetic characteristics matching free space. Since the simulation occurred at a single frequency of $1.6 \mathrm{GHz}$, the blood was set as a frequency-independent, nondispersive material with a relative permittivity of 59.128 . This relative permittivity corresponded to average blood sugar levels near $134 \mathrm{mg} / \mathrm{dL}$ when measured at $1.6 \mathrm{GHz}$ [41]. Figure 30 displays the physical implementation of the design with each material layer identified by color as well as the four input ports.

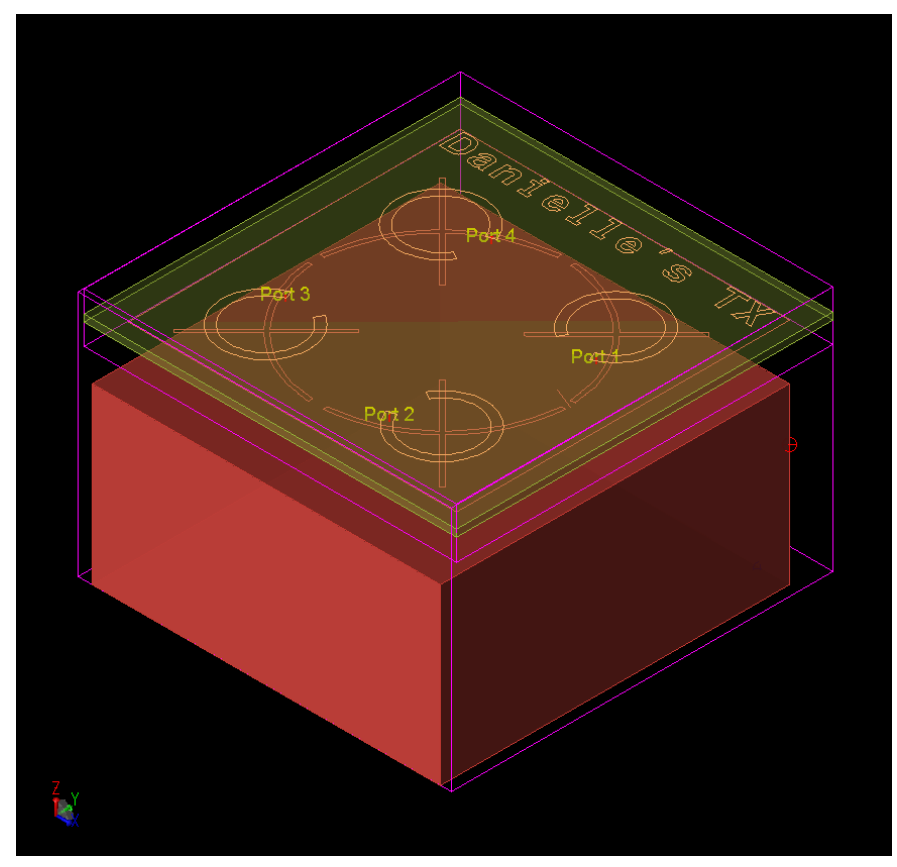

Figure 30: Simulation setup of full slot array antenna and $4 \mathrm{~cm}$ layer of blood (red) separated by $1 \mathrm{~cm}$ of air (unshaded). 
The phase of input port 1 was incremented by 90 degrees at a time to observe the effect of changing a single input's phase. When observing the magnetic field at the bottom of the blood layer, changing the phase to 90 degrees displayed stronger directionality and a more precise output field location than a phase shift of 180 degrees and 270 degrees. The bottom of the blood layer was chosen as the receiving end since it was physically the furthest from the antenna and therefore measured the worst-case field propagation in the simulation. Figure 31 shows the effect of changing the input phase on the magnetic field at the base of the blood layer. The warm red colors indicate stronger magnetic fields, which were chosen as an analysis parameter for its effect on received signal power.

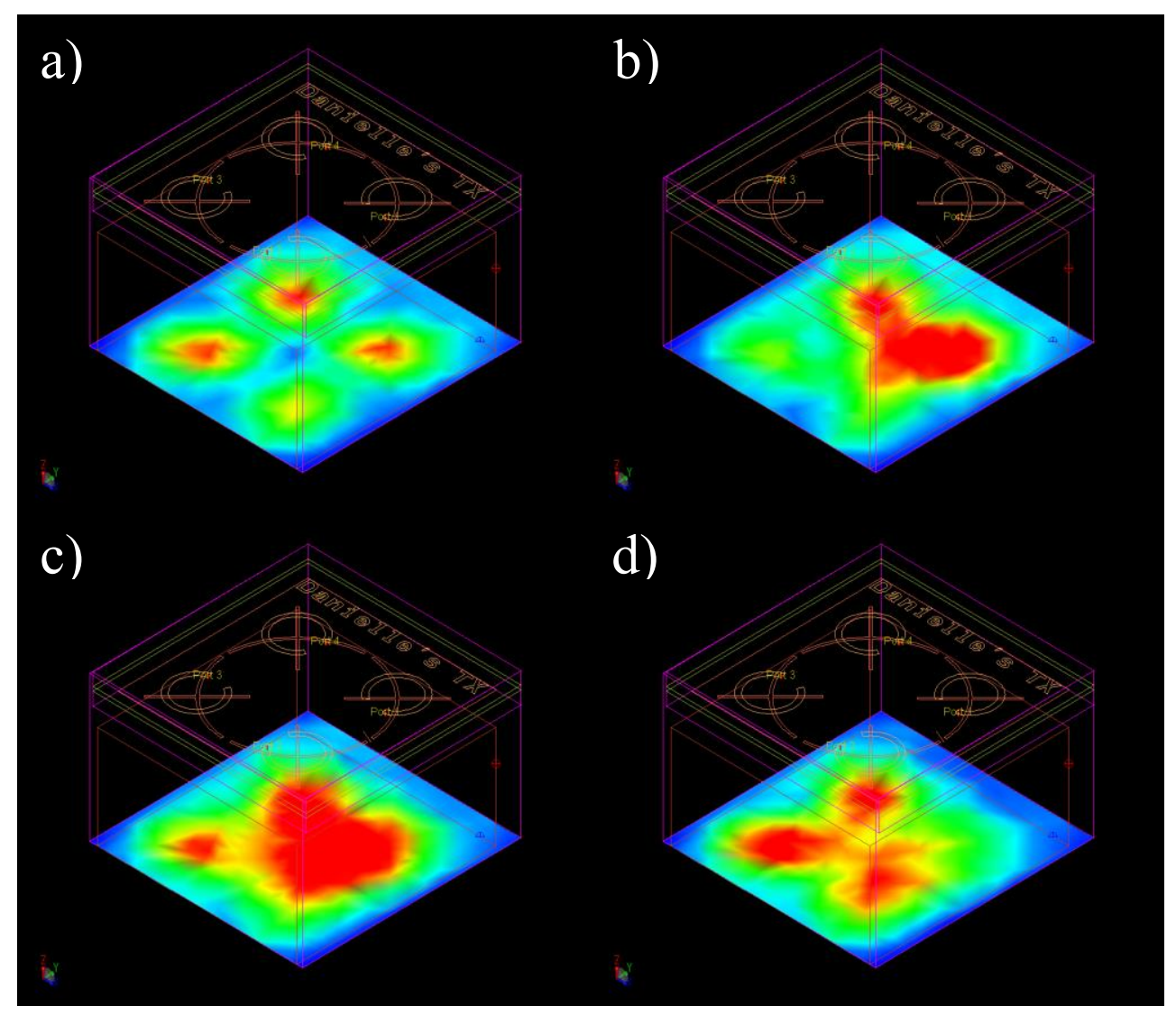


Figure 31: Changing magnetic field magnitudes under the $4 \mathrm{~cm}$ layer of blood after incrementing the phase of input port 1 by 90 degrees ( 0 degrees, 90 degrees, 180 degrees, 270 degrees, respectively).

Further simulations were completed to determine the best phases of all four input ports for optimizing output directionality. Input port 1 remained fixed at 90 degrees due to its maximum directionality as previously described. Each remaining input port was then individually phase-shifted by 90 degree increments, and the received magnetic fields were observed. Figure 32 displays the resulting magnetic field magnitudes at the base of the blood layer for each configuration, which helped narrow down the best setup for maximum directionality. The parameters for performance analysis were the surface area of the highest received energy and the angle of field propagation relative to the center of the antenna. It was desired to achieve strong field concentration at the smallest angle relative to the center of the antenna while maintaining a relatively large surface area, as seen in the first image of Figure 32(a). Therefore, the optimal configuration chosen was a 90 degree phase shift for both input ports 1 and 2 while maintaining no phase change for ports 3 and 4 . 

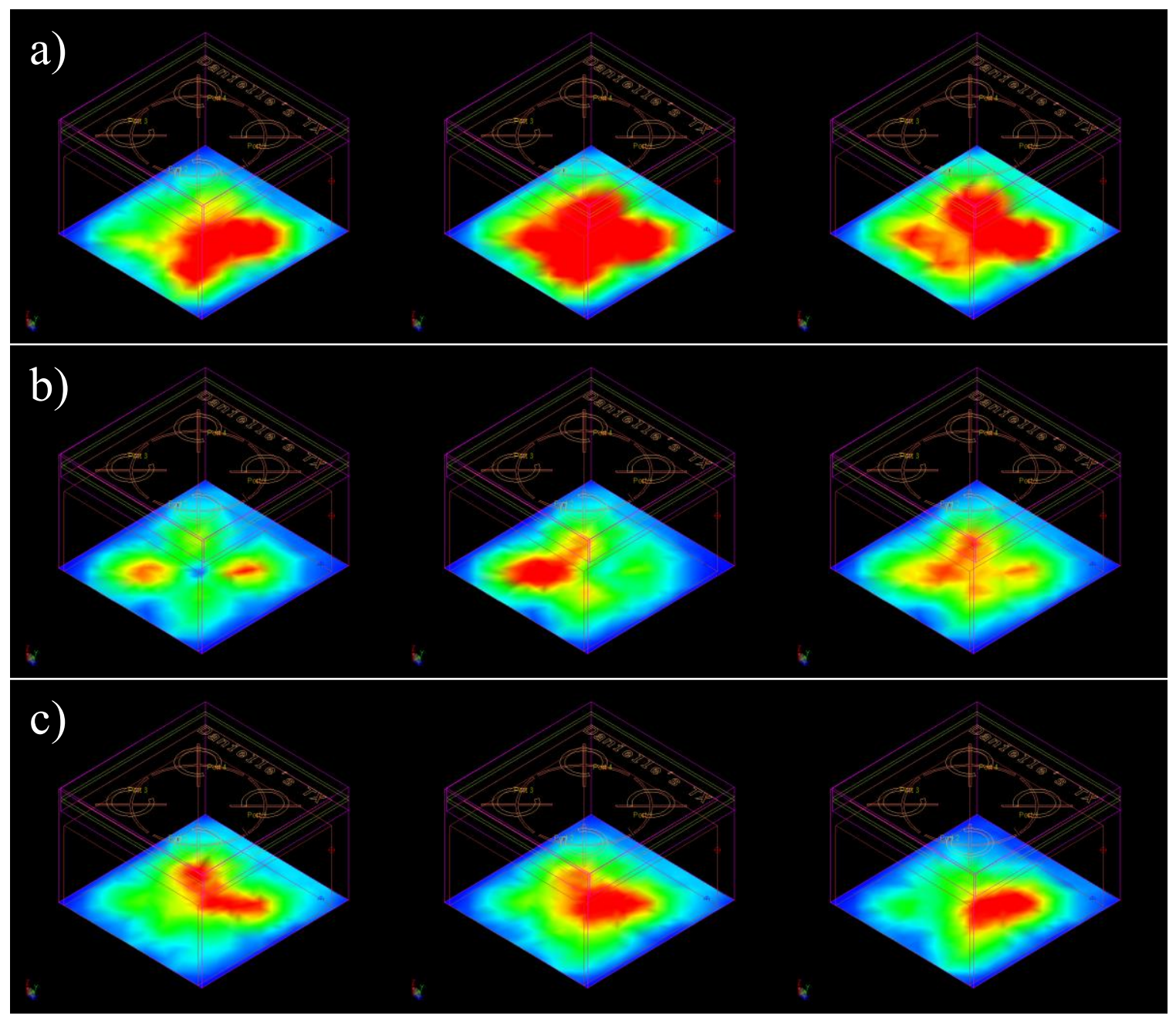

Figure 32: Varying the input phases of the remaining three ports (ports 2, 3, and 4, respectively) by 90 degrees shows the effect of the input phase on the magnetic fields at distances near $5 \mathrm{~cm}$ away.

Additional input configurations were simulated in which three or all four input ports were phase shifted in 90 degree increments at once. Although they produced greater surface areas of high field concentrations at the receiving end, these results showed much less directionality in comparison to the results in Figure 32. The optimal input setup was therefore chosen to match the parameters as described in Table 8.

Table 8: Input port setup determined for optimal directionality per simulation results. 


\begin{tabular}{|c|c|c|}
\hline Port & Weight & Phase [deg] \\
\hline 1 & 1 & 90 \\
\hline 2 & 1 & 90 \\
\hline 3 & 1 & 0 \\
\hline 4 & 1 & 0 \\
\hline
\end{tabular}

The current density of the optimal input setup was also examined to analyze the anticipated decrease in signal strength after propagating through blood. Figure 33 shows the magnitude of the current density at the boundary between air and blood and the receiving end. It is evident that the current density closest to the antenna is much higher than the current density after travelling through blood.

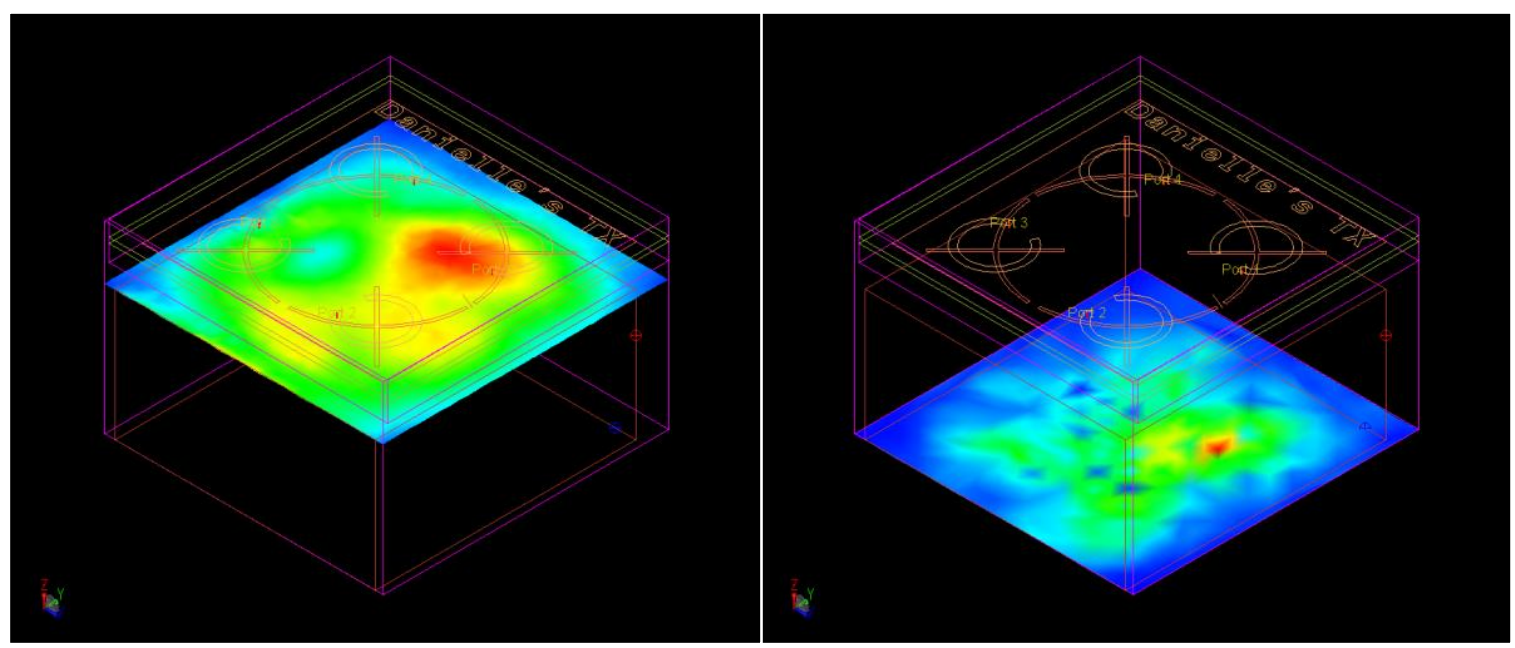

Figure 33: The magnetic field at the interface between air and blood displays the direction of the strongest current density (left) and the resulting current density at the bottom surface of the blood layer (right).

A closer look into the magnetic field propagation through the layer of blood demonstrated the constructive interference of radiating fields from each input. Figure 34 shows time-based snapshots of the magnetic field propagating through the blood layer every 30 degrees of a 360 degree animation. The high concentrations of magnetic field radiation are again shown by the red colors, and the blue colors represent little to no field 
energy. It can be seen that the constructive interference of each input allows for stronger field propagation through greater depths of volume, thus producing greater received fields. These simulations matched the expectations of the antenna design and performance previously described by Ho et. al.

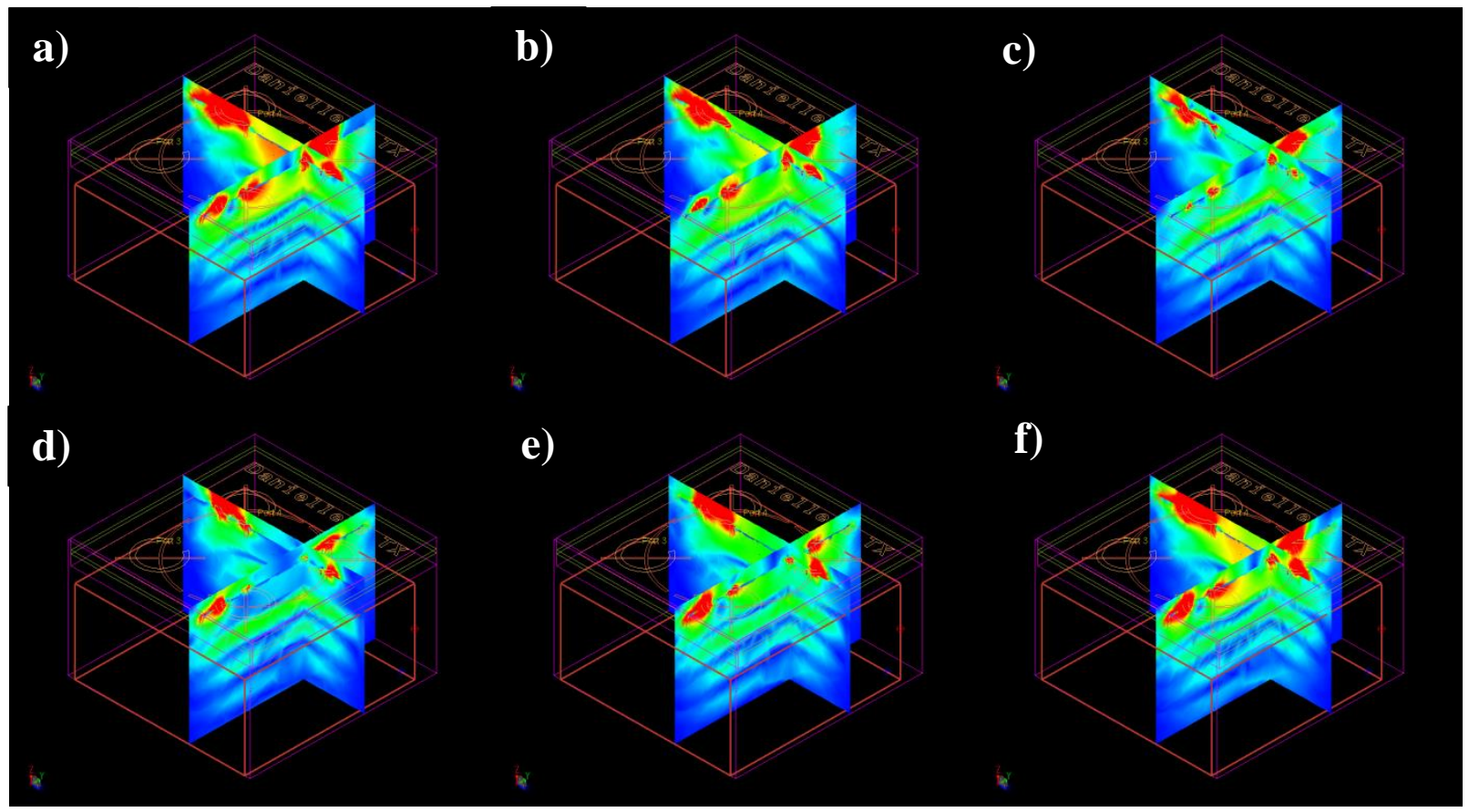

Figure 34: Snapshots of the magnetic field propagating through the blood layer at the intersection of largest current density.

\subsubsection{Three-Dimensional Analysis of Slot Array Antenna Propagating through}

\section{Biological Tissue}

After determining the input setup for optimal directionality, simulations for the antenna's intended application through the biceps brachii were completed. A 3-D model was developed to represent all the layers of biological materials assumed to come into contact with the radiating fields. These layers included skin, blood, fat, and muscle, all of which were sized to match a female in adulthood for future testing purposes. Three different models were also created for this specified biological material structure, but 
each with a different level of blood glucose. The simulated blood glucose levels ranged from $134 \mathrm{mg} / \mathrm{dL}$ (regular), $95 \mathrm{mg} / \mathrm{dL}$ (medium), and $72 \mathrm{mg} / \mathrm{dL}$ (low), and the layers were again parameterized to match an electrically isotropic, nondispersive material with magnetic characteristics matching free space. Table 9 summarizes the physical and electrical properties of each layer, and Figure 35 shows the resulting 3-D model in EMPro.

Table 9: Setup of layers simulated with their thicknesses and electrical parameters. The three types of blood were simulated one by one in combination with all other layers.

\begin{tabular}{|c|c|c|c|}
\hline Material & Thickness & $\begin{array}{c}\text { Conductivity } \\
\text { at } \mathbf{1 . 6} \mathbf{~ G H z}[\mathbf{S} / \mathbf{m}]\end{array}$ & $\begin{array}{c}\text { Relative Permittivity } \\
\text { at } \mathbf{1 . 6} \text { GHz }\end{array}$ \\
\hline Skin & $3 \mathrm{~mm}$ & 1.135 & 44.213 \\
\hline Blood (Regular) & $2 \mathrm{~mm}$ & 1.9117 & 59 \\
\hline Blood (Medium) & $2 \mathrm{~mm}$ & 1.9117 & 63 \\
\hline Blood (Low) & $2 \mathrm{~mm}$ & 1.9117 & 65 \\
\hline Fat & $2 \mathrm{~mm}$ & 0.071352 & 5.3718 \\
\hline Muscle & $3 \mathrm{~cm}$ & 1.2368 & 53.821 \\
\hline Fat & $2 \mathrm{~mm}$ & 0.071352 & 5.3718 \\
\hline Blood (Regular) & $2 \mathrm{~mm}$ & 1.9117 & 59 \\
\hline Blood (Medium) & $2 \mathrm{~mm}$ & 1.9117 & 63 \\
\hline Blood (Low) & $2 \mathrm{~mm}$ & 1.9117 & 65 \\
\hline Skin & $3 \mathrm{~mm}$ & 1.135 & 44.213 \\
\hline
\end{tabular}




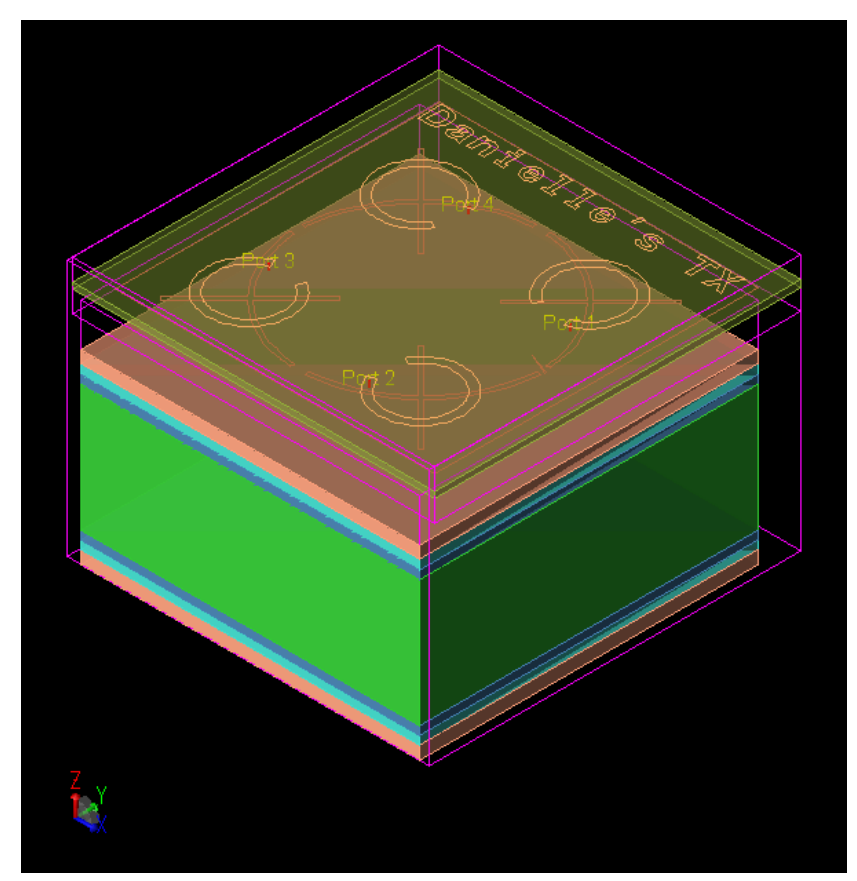

Figure 35: Physical setup for simulation environment. The antenna was separated by $1 \mathrm{~cm}$ of air, followed by $3 \mathrm{~mm}$ of skin, $2 \mathrm{~mm}$ of blood, $2 \mathrm{~mm}$ of fat, $3 \mathrm{~cm}$ of muscle, $2 \mathrm{~mm}$ of fat, $2 \mathrm{~mm}$ of blood, and $3 \mathrm{~mm}$ of skin.

The simulations produced magnetic and electric field magnitudes as well as the current density magnitude at the receiving plane of the outer skin layer. Field magnitudes corresponded to the expected behavior due to the inverse relationship between blood glucose and dielectric permittivity. As the glucose levels decreased, all three magnitude measurements increased due to greater energy storage capabilities. Table 10 summarizes the resulting field magnitudes in relation to the blood glucose levels; and Figure 36, Figure 37, and Figure 38 show the changes in field magnitudes based on blood glucose levels.

Table 10: EMPro simulation results demonstrated the inverse relationship between glucose and received current density, magnetic field, and electric field. 


\begin{tabular}{|c|c|c|c|c|c|}
\hline Blood & $\begin{array}{c}\text { Glucose Level } \\
{[\mathbf{m g} / \mathbf{d L}]}\end{array}$ & $\begin{array}{c}\text { Relative } \\
\text { Permittivity }\end{array}$ & $\begin{array}{c}|\mathbf{J}| \\
{\left[\mathbf{A} / \mathbf{m}^{2}\right]}\end{array}$ & $\begin{array}{c}|\mathbf{H}| \\
{[\mathbf{A} / \mathbf{m}]}\end{array}$ & $|\mathbf{E}|[\mathbf{V} / \mathbf{m}]$ \\
\hline Regular & 134 & 59 & 0.03912 & 0.03546 & 2.104 \\
\hline Medium & 95 & 63 & 0.04218 & 0.04133 & 2.105 \\
\hline Low & 72 & 65 & 0.04368 & 0.04785 & 2.114 \\
\hline
\end{tabular}

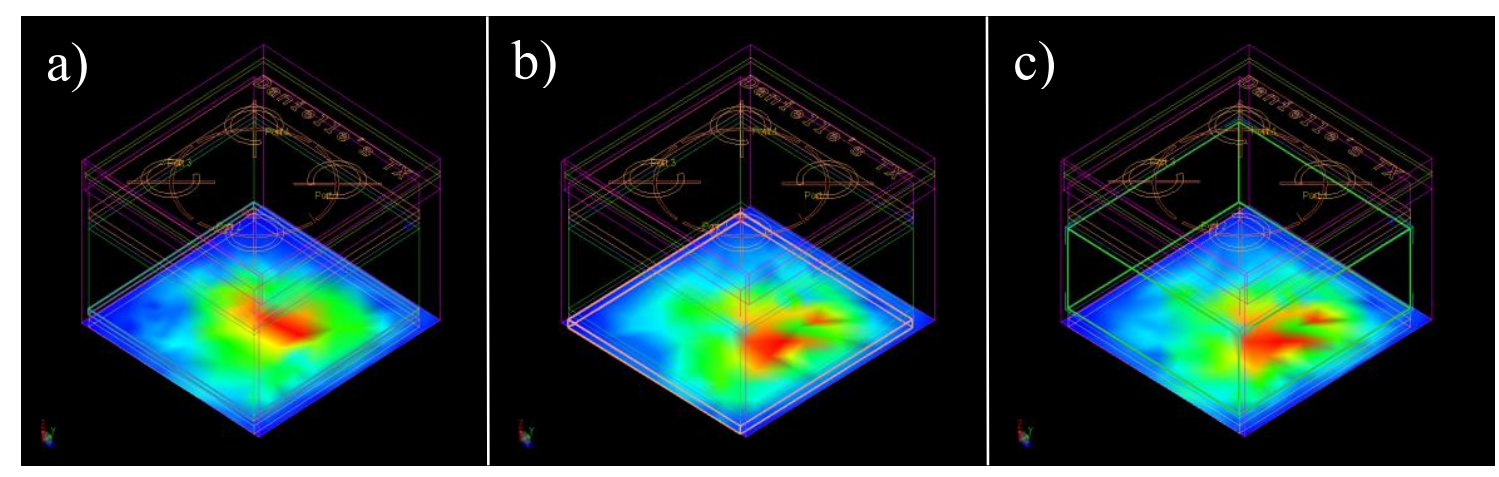

Figure 36: Magnitude of the current density on the receiving side of the simulated biceps based on the optimal input phase settings. The simulations are in order from highest to lowest blood sugar, and the resulting magnitudes are 0.03912, 0.04202, and 0.04368

$$
\mathrm{A} / \mathrm{m}^{2} \text {, respectively. }
$$

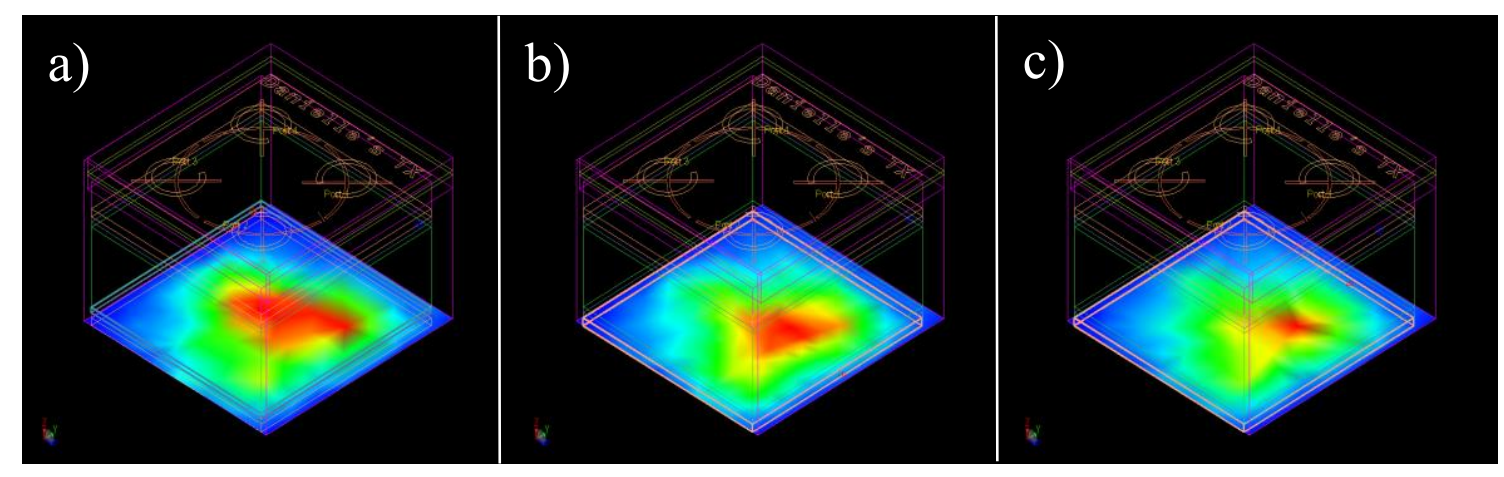

Figure 37: Magnitude of the magnetic field on the receiving side of the simulated biceps based on the optimal input phase settings. The simulations range from the (a) highest blood sugar level with $0.03546 \mathrm{~A} / \mathrm{m}$, to (b) medium blood sugar level with $0.04133 \mathrm{~A} / \mathrm{m}$, and (c) lowest blood sugar level with $0.04785 \mathrm{~A} / \mathrm{m}$. 


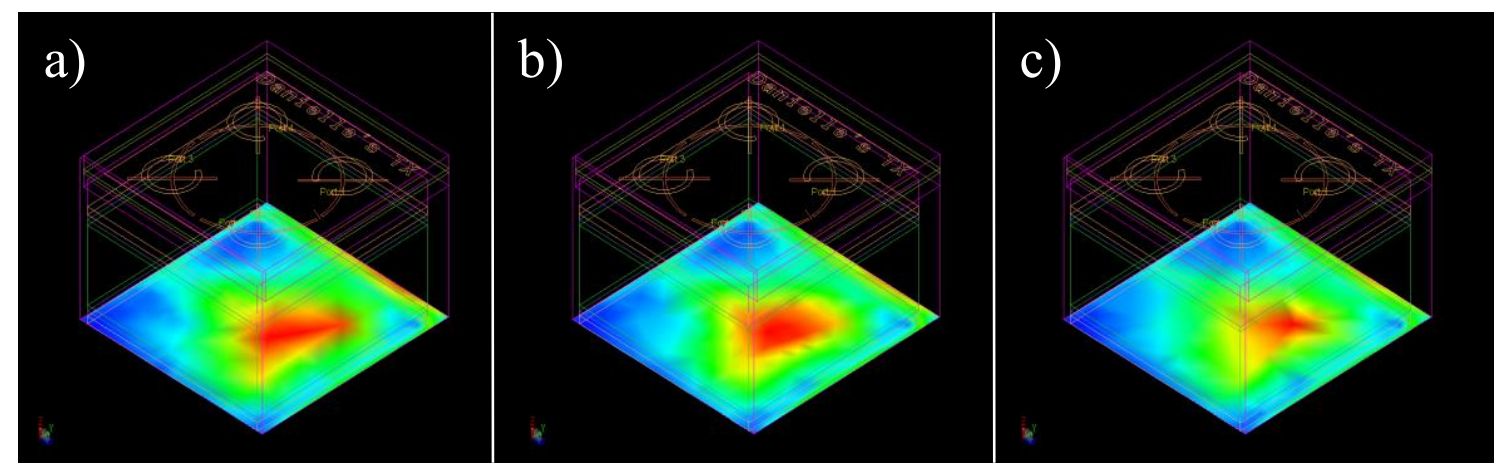

Figure 38: Magnitude of the electric field on the receiving side of the simulated biceps based on the optimal input phase settings. The simulations range from the (a) highest blood sugar level with $2.104 \mathrm{~V} / \mathrm{m}$, to (b) medium blood sugar level with $2.105 \mathrm{~V} / \mathrm{m}$, and (c) lowest blood sugar level with $2.114 \mathrm{~V} / \mathrm{m}$.

When looking at the top of the antenna pointing into the biceps brachii, the magnitude of the magnetic field at the boundary between the $1 \mathrm{~cm}$ air gap and skin provides a general area of maximum expected radiation. Figure 39 shows the magnetic field at this boundary, which was used to pinpoint the location of the strongest field waves travelling through the anticipated biological layers.

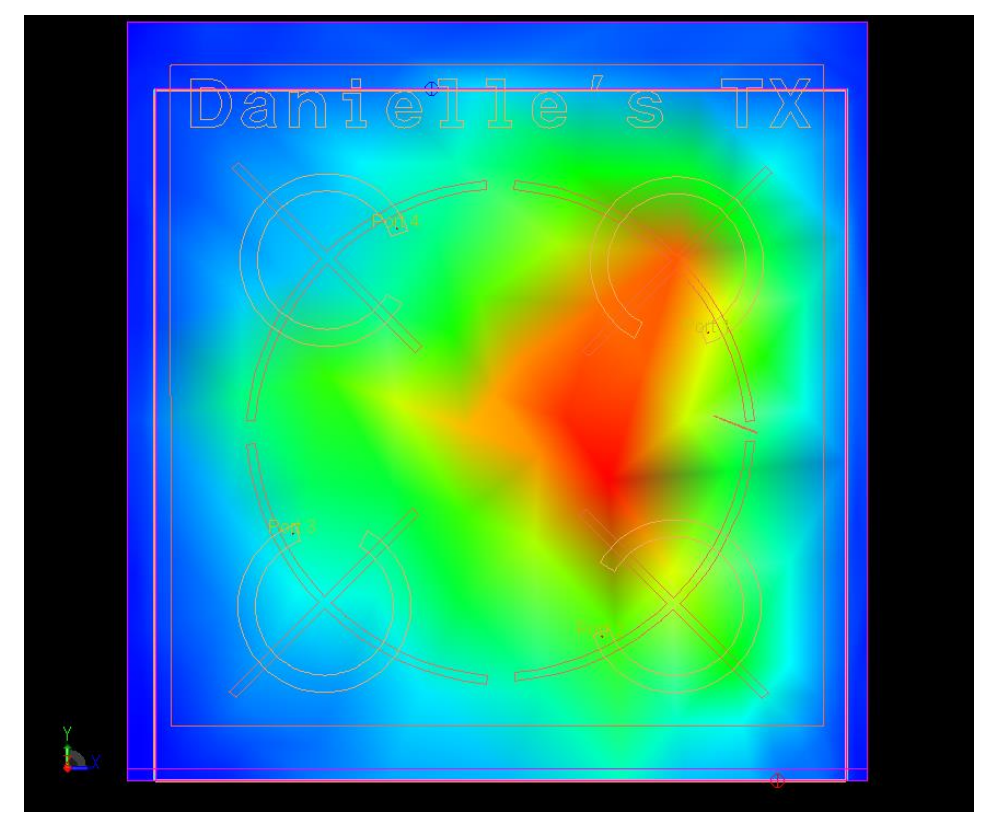


Figure 39: Based on the simulation for the medium level of blood glucose, the magnitude of the magnetic field at the surface of the skin furthest from the antenna determined a generic location for the output.

In a series of time-based snapshots, Figure 40 shows the magnetic field intensities throughout the tissue layers at 30 degree increments. Again, it can be seen that field intensity easily reaches the receiving skin layer due to the constructive interference from all four inputs. These images also provided an expected location for the strongest field presence that would ideally receive the strongest field energies.

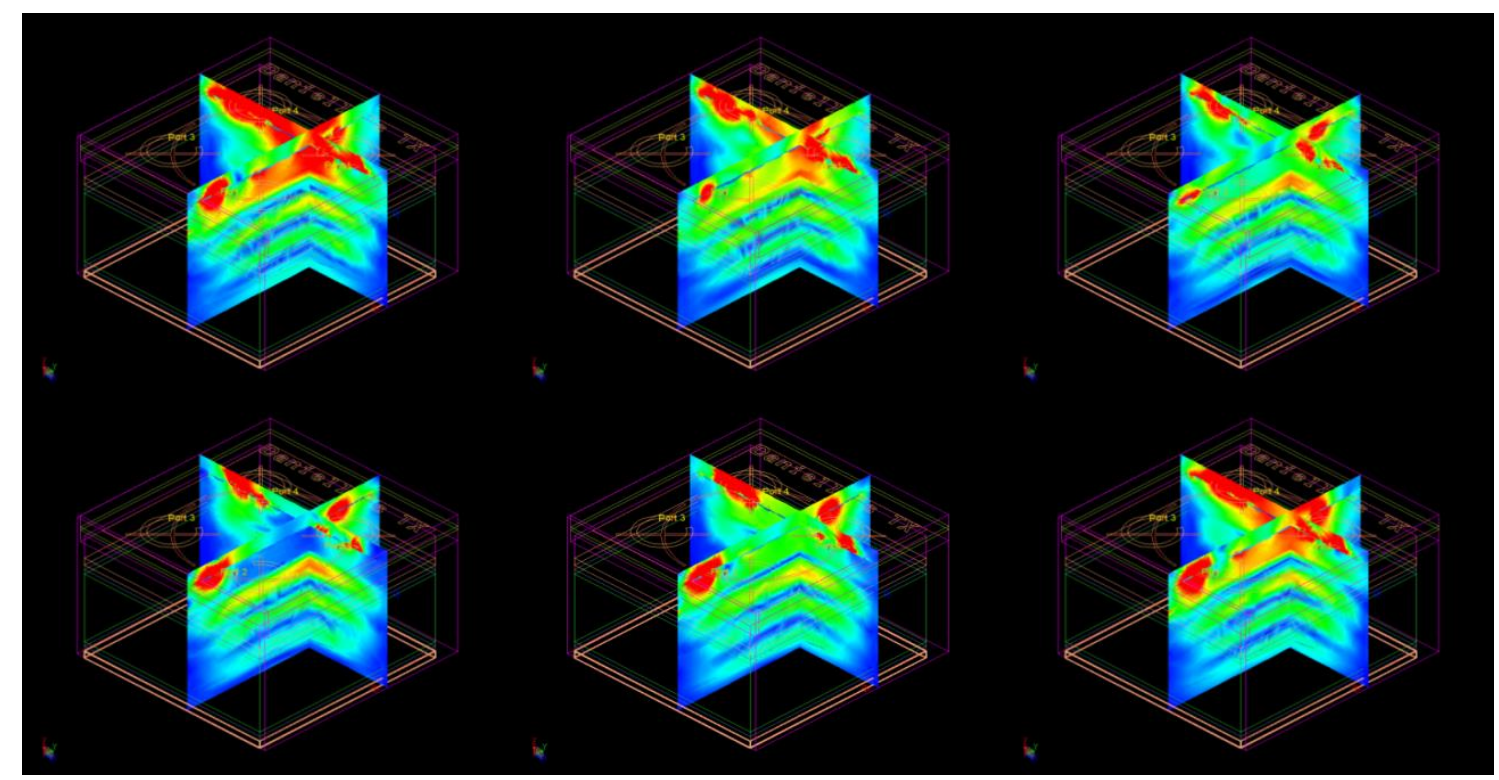

Figure 40: Animation stills of the propagating magnetic field through all the described biological layers. The animation images are captured in increments of 30 degrees and range from 0 to 150 degrees.

\subsection{Receiver Design for Slot Array Antenna Transmitter}

Upon completing simulations for the antenna's expected behavior, a receiver design was required to translate blood glucose levels into received power. A loop antenna was chosen to act as the receiving antenna for easier implementation with the 
receiving module. It was decided that the loop antenna would have a perimeter of the operating frequency's wavelength in free space with the option of additional coil windings to fine tune the resonant frequency if needed. The antenna would be constructed with copper magnet wire provided in the Microwave Engineering Lab on Cal Poly's campus primarily due to its immediate availability. Magnet wire was also desired for its high sensitivity to magnetic fields resulting from the very thin insulation layer. Constructing the antenna was determined to occur during the testing phase of the development cycle.

The requirements for the processing end of the receiver included direct power measurements in $\mathrm{dBm}$ for an input signal at $1.6 \mathrm{GHz}$ when placed on the opposite side of the biceps brachii. To do so, an RF detector receiver was designed by Dawei Zhang [37]. This receiver used the LT 5534 integrated circuit by Linear Technology due to its powerful features. The LT 5534 is an RF detector that operates from $50 \mathrm{MHz}$ to $3 \mathrm{GHz}$ and converts a $60 \mathrm{~dB}$ dynamic range RF signal into DC voltage based on a linear scale of $44 \mathrm{mV} / \mathrm{dB}$, as specified by the data sheet [42]. The ability to receive signals as low as $60 \mathrm{~dB}$ was the primary advantage in choosing this device since it corresponded to $1 \mu \mathrm{W}$ of received power. With expected received power measurements in the $\mathrm{mW}$ range, as seen in simulations, this RF detector was deemed capable of receiving slight changes in signal strength due to changes in glucose.

The accompanying demonstration board offered by Linear Technology was also chosen to implement the LT $5534 \mathrm{RF}$ detector. The main advantage in choosing to implement the demonstration board was to ease the connection of the surface mounted LT 5534 integrated circuit with the remaining through-pin circuitry of the receiver. The 
LT 5534ESC6 demonstration board also incorporated an SMA connector for the direct RF input to the LT 5534 integrated circuit and signal conditioning circuitry. Furthermore, the demonstration board provided the direct voltage output corresponding to the received signal power, operated on a range of $2.7 \mathrm{~V}$ to $5.25 \mathrm{~V}$, and incorporated an output enable pin if desired.

To ease the data collection process, an Arduino Uno development board was connected to the receiver to directly measure the received signal strength intensity [43]. The Arduino Uno was programmed with functions to directly convert the DC voltage into a magnitude of $\mathrm{dBm}$ without any need for a spectrum analyzer. An attached LCD display was chosen to display the received signal strength in $\mathrm{dBm}$ based on the calculations determined by the Arduino's processor. 


\section{Chapter 4: Glucose Sensor Test Setup and Results}

\subsection{System Assembly}

The entire glucose sensing system was set up with the individual parts described in Chapter 3 and pictured in Figure 41. The flow of the system began with two input signals for the local oscillator and RF input into the mixer, of which the output was filtered to isolate the desired $1.6 \mathrm{GHz}$ signal. This was followed by the 4-way Wilkinson power divider to provide a signal source to each of the four inputs of the slot array antenna. Upon transmission, the received signal was captured by a custom sensor. The sensor was realized in a loop antenna shaped to receive $1.6 \mathrm{GHz}$ signals and made from magnet wire. The received signal strength was then converted into a DC voltage with an RF detector and displayed in $\mathrm{dBm}$ on an LCD attached to an Arduino Uno processing board.

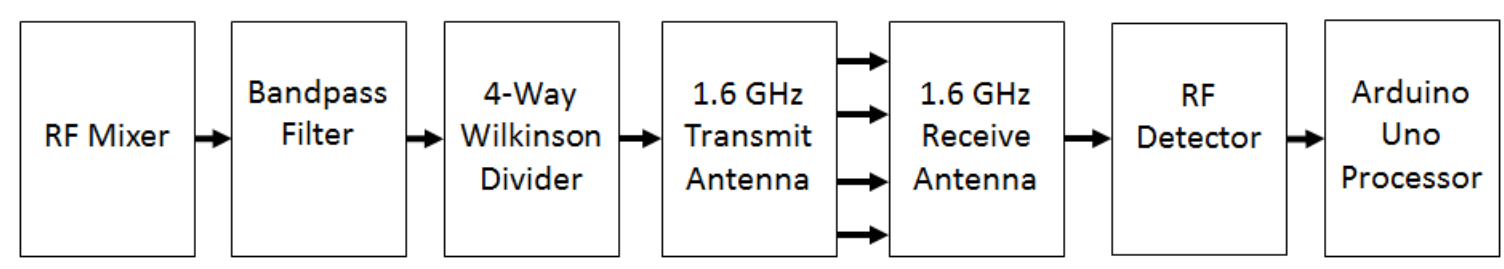

Figure 41: Block diagram of the entire sensing system, beginning with the RF mixer and ending with the Arduino Uno Processor.

\subsection{Testing the Signal Transmitter}

When testing the signal transmitter, the best approach was to characterize each component of the transmitting antenna system. This included the RF mixer, bandpass filter, Wilkinson divider, and the slot array antenna. An overall method of characterization was measuring the scattering parameters of each component since they 
were all RF devices. Additional characterization was also completed with an Agilent N9000A CXA Signal Analyzer when necessary.

\subsubsection{RF Mixer and Bandpass Filter Test Setup and Results}

The RF mixer, as pictured in Figure 42, was the first stage of the signal transmitter setup. A $1000 \mathrm{MHz}$ local oscillator signal at $10 \mathrm{dBm}$ was generated by the Agilent 33210A Arbitrary Waveform Generator and mixed with a $600 \mathrm{MHz}$ RF signal also at $10 \mathrm{dBm}$ and generated by a Fluke 6060B RF Signal Generator. The mixer board was designed and created by Dawei Zhang for the purpose of this glucose sensor, as shwon in Figure 42 [37].

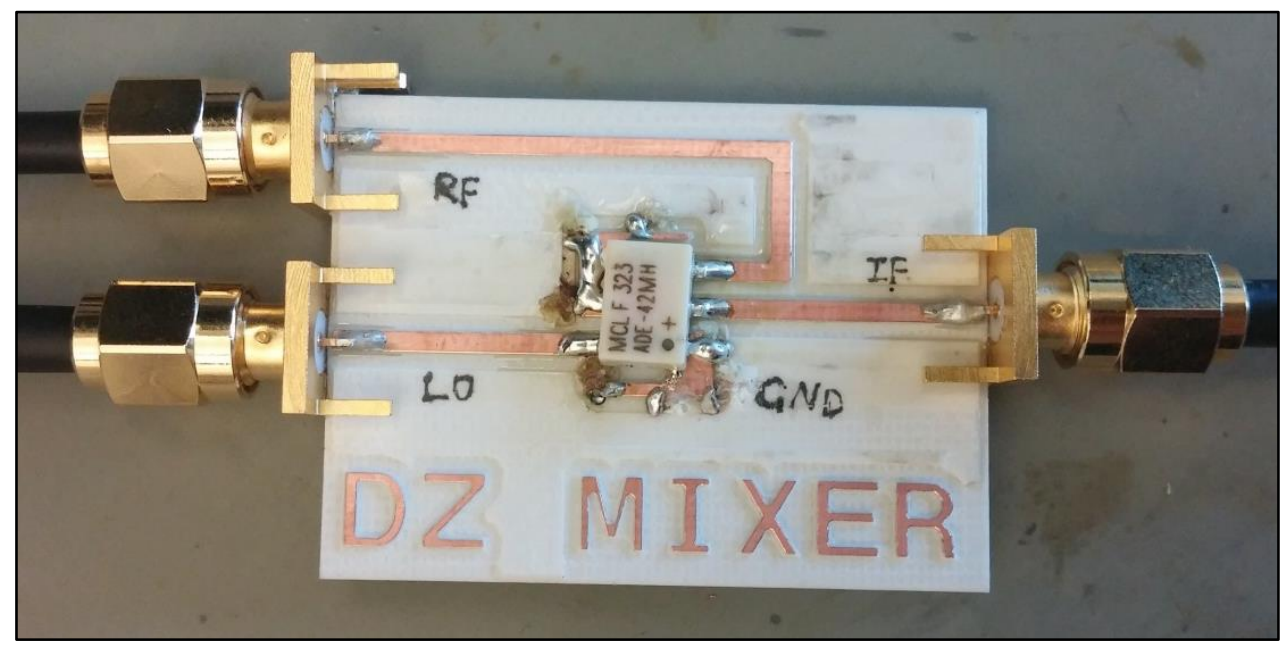

Figure 42: RF mixer implementing a local oscillator and RF input signal used to create a $1.6 \mathrm{GHz}$ output for the slot array antenna.

The resulting output of the RF mixer was then filtered with the bandpass filter, also designed by Dawei Zhang. This was required to remove the unwanted harmonics at all frequencies other than $1.6 \mathrm{GHz}$. The filter focused on a $5^{\text {th }}$-order design method used to create a sharp cutoff near $1.6 \mathrm{GHz}$ rather than a lower order design that might not 
attenuate the unwanted harmonics. Figure 43 shows the layout of the bandpass filter, and the five stub patches that indicate each order of the design.

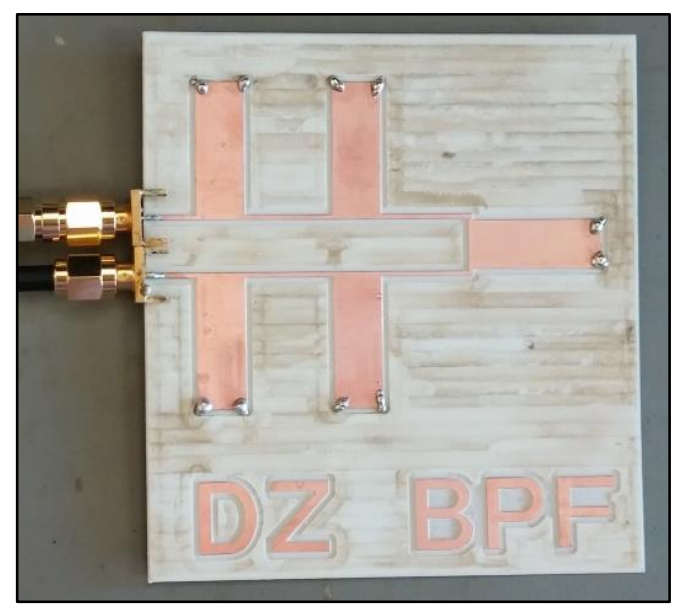

Figure 43: Bandpass filter centered at $1.6 \mathrm{GHz}$ used to remove harmonics from the output of the RF mixer.

The resulting 1.6 GHz signal was characterized using the Agilent N9000A CXA Signal Analyzer. Figure 44 shows the signals produced by the RF mixer and bandpass filter across $1.3 \mathrm{GHz}-1.9 \mathrm{GHz}$. It is important to note that sideband signals exist at 1.4 $\mathrm{GHz}$ and $1.8 \mathrm{GHz}$; however, this was expected and dampened by the bandpass filter. The sideband signals at $1.4 \mathrm{GHz}$ and $1.8 \mathrm{GHz}$ were significantly weaker than the $-8.14 \mathrm{dBm}$ signal at the center frequency by at least $20 \mathrm{dBm}$. 


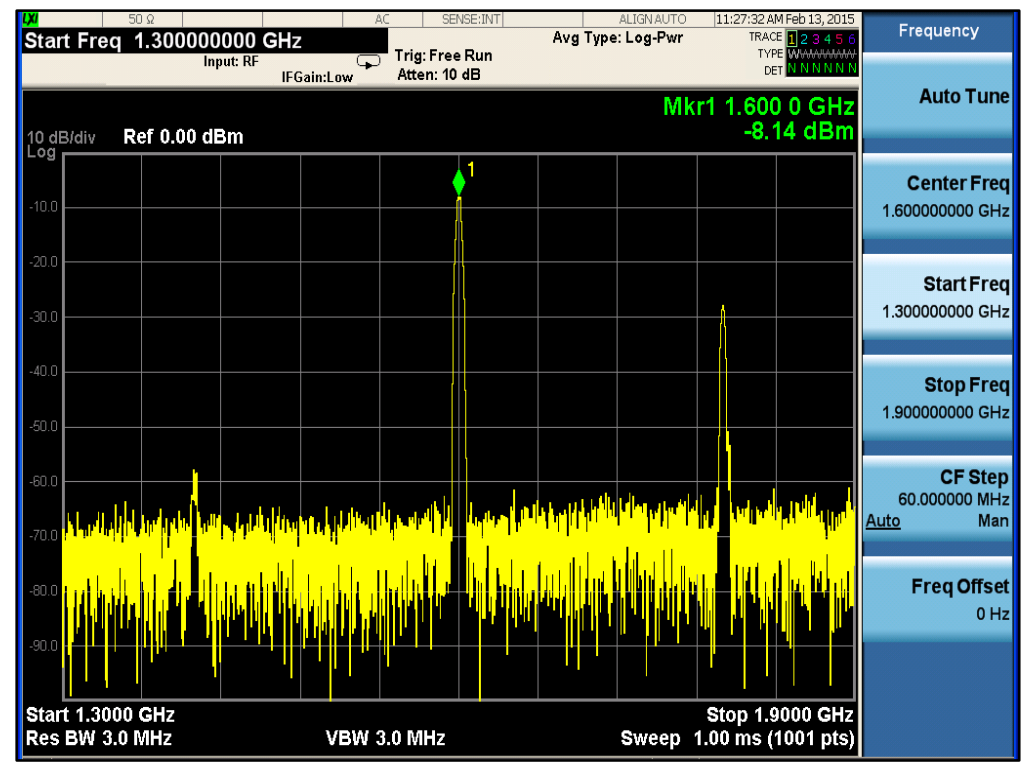

Figure 44: Due to the nature of the RF mixer, multiple signals were generated across 1.3 GHz-1.9 GHz, but filtered well with the designed bandpass filter.

\subsubsection{Wilkinson Power Divider Test Setup and Results}

The 4-output Wilkinson power divider, as seen in Figure 45, was characterized based on two metrics: the measured scattering parameters and the signal generated at each output. The measured scattering parameters focused on the forward and reverse paths between the single input and each of the four outputs. Table 11 summarizes the scattering parameters as measured by the Anritsu 4622B Vector Network Analyzer. The results matched well to the designed values with nearly $-6 \mathrm{~dB}$ of loss and nearly equal phase shift at each output. 


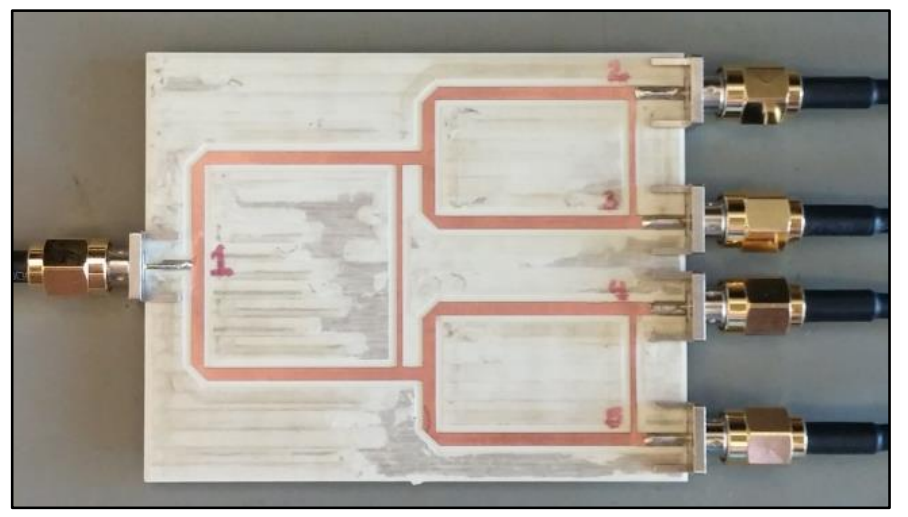

Figure 45: 4-output Wilkinson power divider designed for splitting a single input into four different outputs with equal loss.

Table 11: Measured parameters of the 4-way Wilkinson Power divider. Each measured parameter from the input to output were near $-6 \mathrm{~dB}$, as desired by the design.

\begin{tabular}{|c|c|c|c|c|c|}
\hline \multicolumn{3}{|c|}{ Forward Parameters } & \multicolumn{3}{c|}{ Reverse Parameters } \\
\hline Parameter & Magnitude [dB] & Phase [degrees] & Parameter & Magnitude [dB] & Phase [degrees] \\
\hline S21 & -5.72 & 40.4 & S12 & -5.86 & 31.3 \\
S31 & -5.45 & 41.2 & S13 & -5.82 & 31.8 \\
\hline S41 & -5.62 & 39.8 & S14 & -6.05 & 30.4 \\
S51 & -5.57 & 40.5 & S15 & -5.91 & 31.2 \\
\hline
\end{tabular}

The power divider was further characterized through measurements with the Agilent N9000A CXA Signal Analyzer. Figure 46 shows the magnitude of each $1.6 \mathrm{GHz}$ signal at the four output ports, which ranged between $-35.01 \mathrm{dBm}$ and $-43.44 \mathrm{dBm}$. The strongest output signals were generated at ports 4 and 5 of the Wilkinson power divider. 


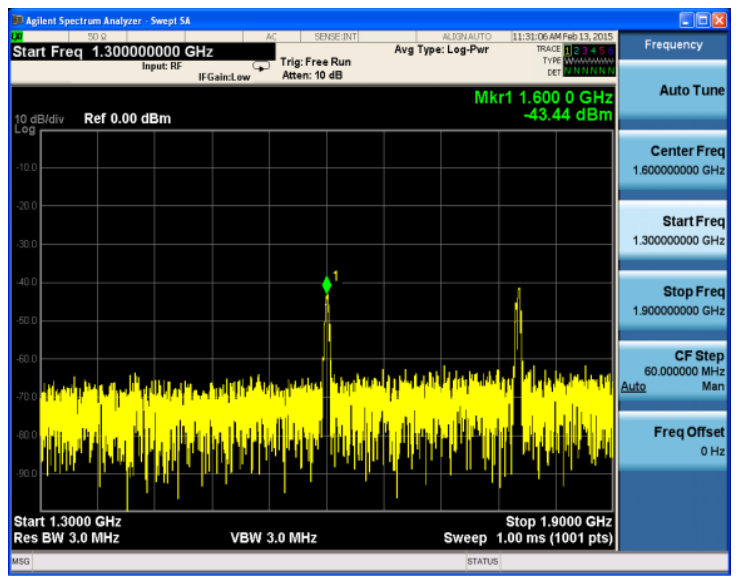

(a)

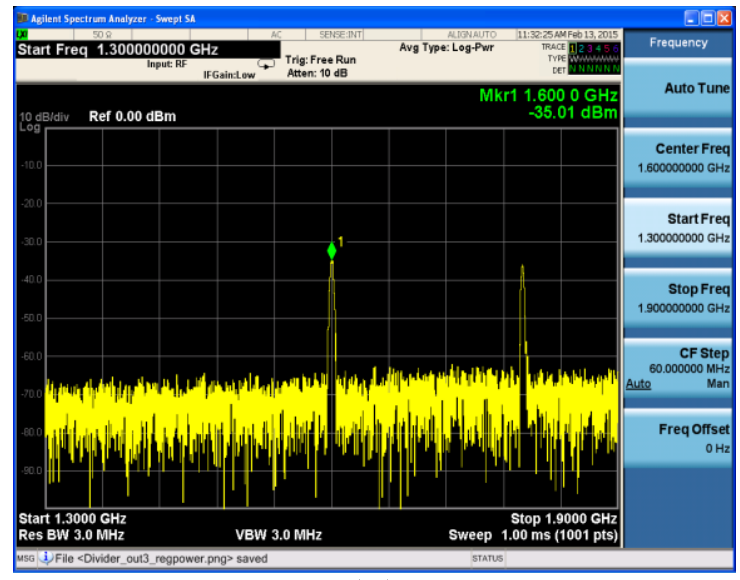

(c)

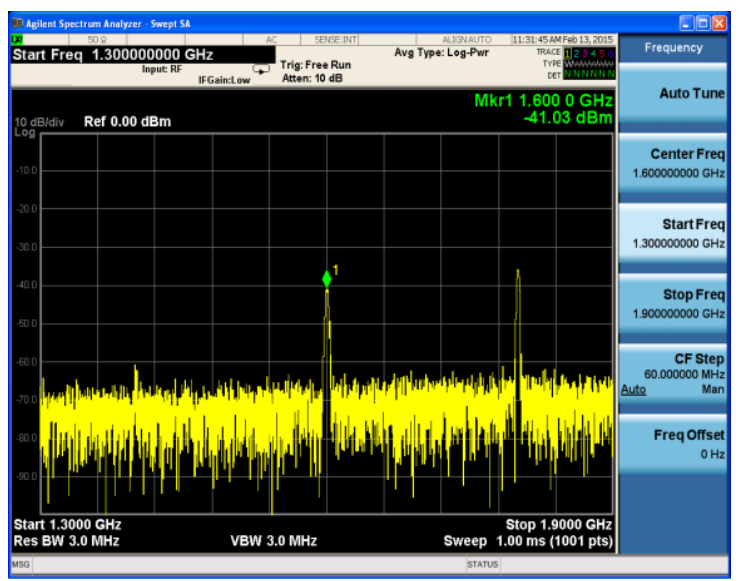

(b)

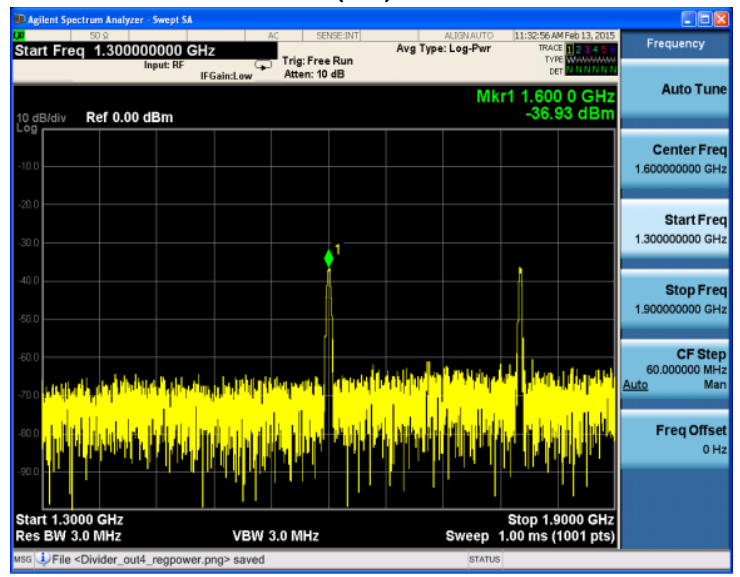

(d)

Figure 46: Measured signals at each of the four output ports of the 4-way Wilkinson divider with $10 \mathrm{dBm}$ RF mixer input signals. Ports 2 through 5 correspond to (a) through (d), respectively.

Although the forward and reverse paths closely matched the desired scattering parameters, these measurements indicated that additional loss incurred at each output port and ultimately degraded signal quality for all outputs. The potential causes for additional loss were signal fringing at the corners of the microstrip traces and coupling between nearby parallel microstrips. Fringing occurs most frequently at sharp corners of microstrip patterns and is associated with the electric field pattern between the charged microstrip and ground plane. At sharp corners, the field lines curve outward and away 
from the shortest path between the microstrip and ground plane, ultimately degrading the field strength and removing energy from the microstrip layer [44]. The multiple sharp corners of the layout provide an understandable reason as to why the output signal strength was significantly lower than the anticipated $-6 \mathrm{~dB}$ of loss.

An additional cause of signal degradation could be the close proximity between parallel microstrip lines, which could cause coupling between the two. Coupled lines change the behavior of the microstrips by transferring energy from one microstrip to another in close proximity, typically for microstrips a quarter-wavelength long. This coupling would alter the expected behavior by possibly removing energy from the microstrips and creating greater reflections from the microstrip resistors [45]. The close proximity between the first vertical resistor microstrip and the vertical separation junctions, as indicated in Figure 47, could possibly couple and change the output signal strength.

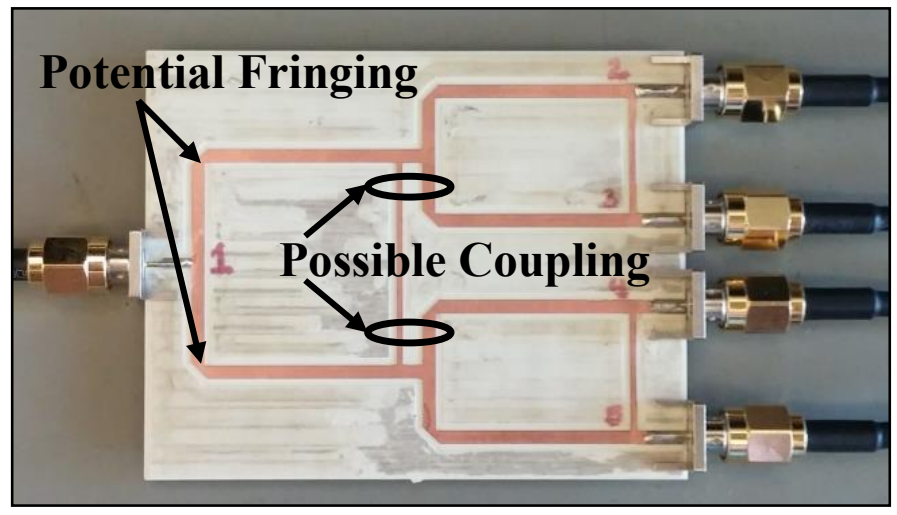

Figure 47: Possible sources of signal loss at each output included potential fringing at sharp corners and coupling between nearby microstrips.

To remedy the significant loss in signal strength from the power divider, the input signals for the RF mixer were increased to their maximum value of $15 \mathrm{dBm}$ per signal. This resultantly increased the output of the mixer and bandpass filter and 
provided a stronger input signal to the Wilkinson power divider. Figure 48 shows the subsequent increase in signal strength at each of the divider's four output ports. This change increased the outputs by nearly $5 \mathrm{dBm}$, and brought the outputs near the range of $-30.85 \mathrm{dBm}$ to $-39.24 \mathrm{dBm}$. These signal values were deemed sufficient since the detector at the receiver had a $-60 \mathrm{dBm}$ sensitivity.

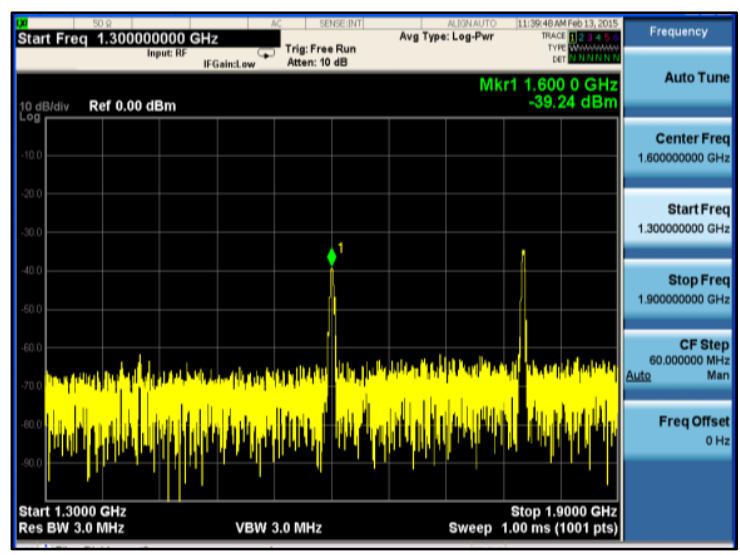

(a)

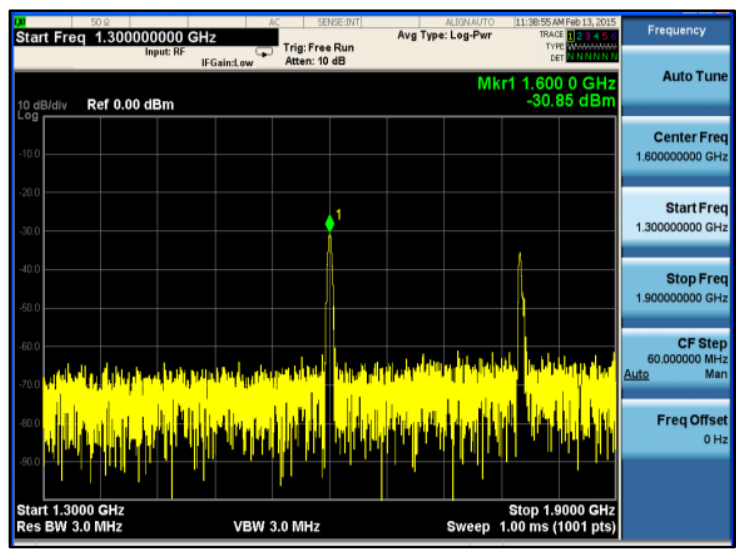

(c)

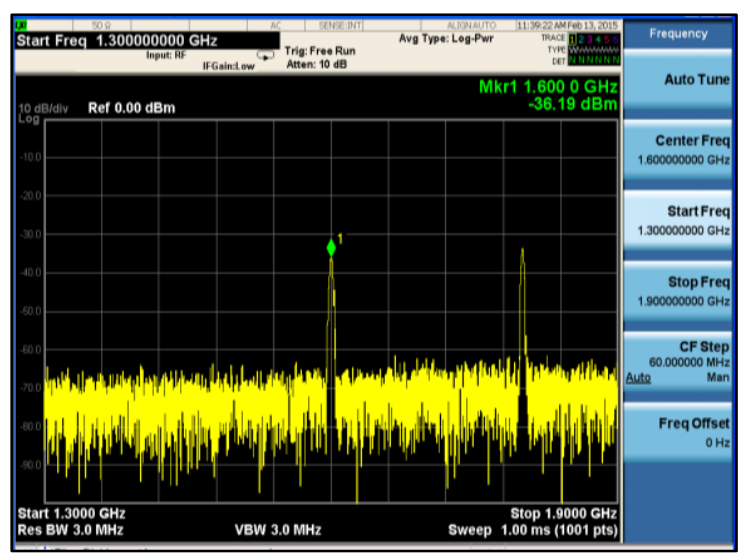

(b)

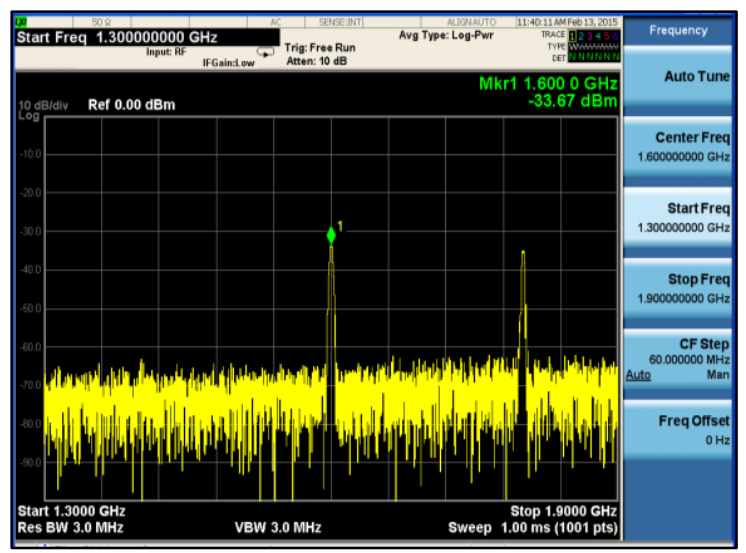

(d)

Figure 48: Measured signals at each of the four output ports of the 4-way Wilkinson divider with $15 \mathrm{dBm}$ RF mixer input signals. Ports 2 through 5 correspond to (a) through (d), respectively.

\subsubsection{Slot Array Antenna Test Setup and Results}


The slot array antenna was characterized by measuring scattering parameters at each input port. Each input port was soldered to a semi-rigid coaxial cable with the conductive center pin fed through the ground plane of the board and up to the microstrip. A physical hole was drilled at the input ports to allow for this signal feed from beneath the board. All input cables were oriented to feed from the same direction, so some of the conductive center pins were curved to reach the microstrip on the top layer. The outer ground shielding of each coaxial cable was also soldered to the ground plane of the board. Figure 49 shows the microstrip layer with coaxial conducting pins soldered to the end of each circular shape as well as the shielding on the cables soldered to the grounded slot layer.

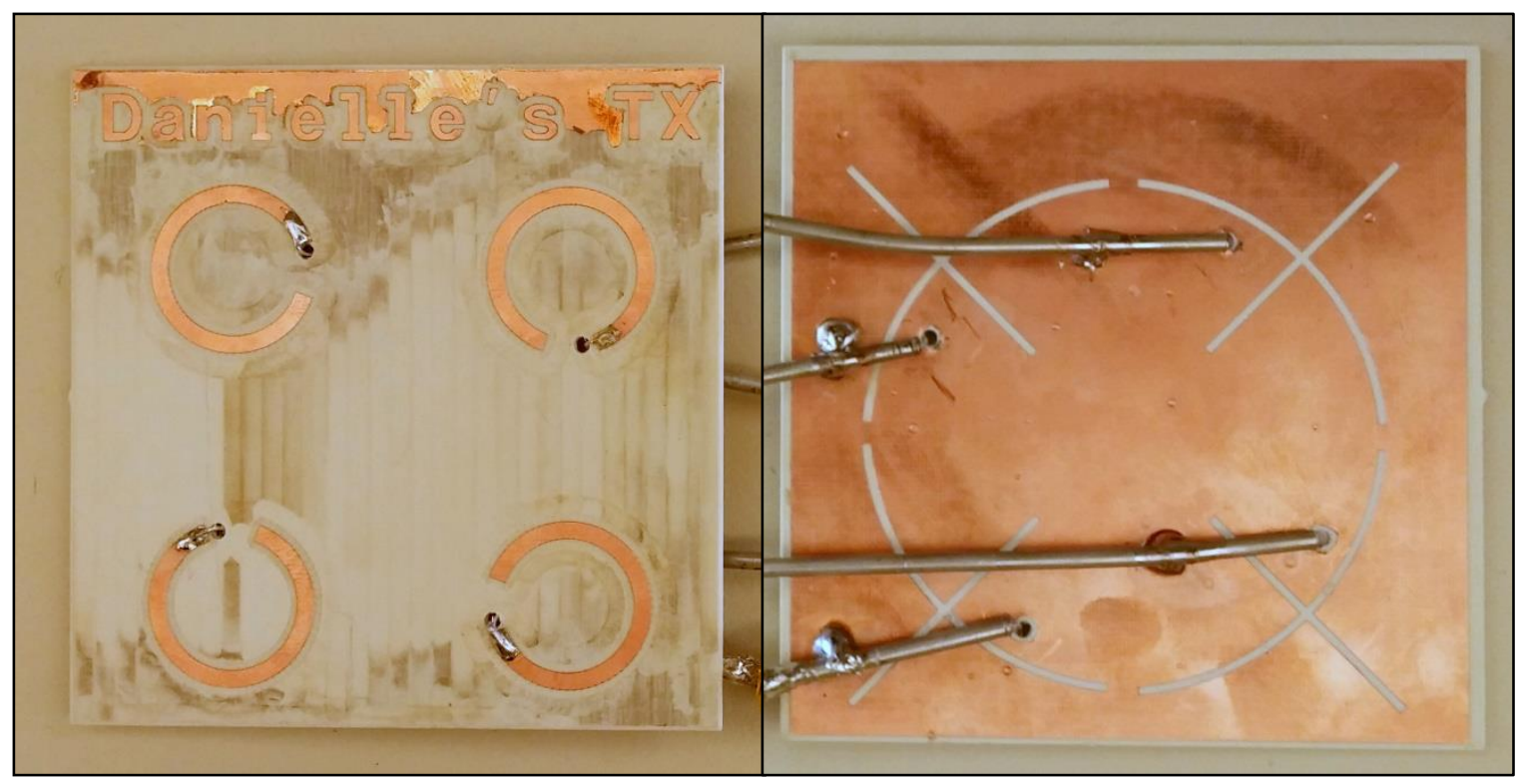

Figure 49: Slot array antenna with connecting coaxial cables soldered to the microstrip layer (left) and ground/slot layer (right).

The connecting coaxial cables soldered to the slot array were cut at a length to match the desired phase shift between the input ports. As determined previously, inputs 1 
and 2 were desired to lead inputs 3 and 4 by 90 degrees. The cables at inputs 1 and 2 were then cut short by a quarter of a wavelength to create this phase shift. The necessary difference in cable length was calculated based on the equation $\lambda=\frac{c}{f * \sqrt{\epsilon_{r}}}$. Since the relative permittivity of the PTFE dielectric in the coaxial cable was 2.1 , the calculated quarter-wavelength was $3.45 \mathrm{~cm}$ for an operating frequency of $1.6 \mathrm{GHz}$. The resulting cable lengths for inputs 1 and 2 were therefore $15.3 \mathrm{~cm}$, and the cable lengths for inputs 3 and 4 were $18.75 \mathrm{~cm}$.

Upon measurements with the Anritsu 4622B Vector Network Analyzer, the scattering parameters for all four inputs did not appear as expected. Measurements for $\mathrm{S}_{33}$ and $\mathrm{S}_{44}$ showed results better than the simulated values with magnitudes of $-34.01 \mathrm{~dB}$ and $-26.2 \mathrm{~dB}$ respectively. However, the measurements for $\mathrm{S}_{11}$ and $\mathrm{S}_{22}$ showed large reflections from the microstrip and resulted in degraded scattering parameters. The measured scattering parameter for $S_{11}$ was $-2.28 \mathrm{~dB}$, and the measured value for $S_{22}$ was $2.04 \mathrm{~dB}$. Attempts at soldering new coaxial cables to these inputs were made and tested, yet the scattering parameters did not improve with these changes.

To offset these issues, inputs 3 and 4 were connected to output ports 4 and 5 of the Wilkinson power divider because they contained the strongest output signals. Since it was proven that inputs 3 and 4 of the slot array antenna were perfectly matched, it was guaranteed that these microstrips would radiate as necessary. The remaining unknown was the capability of propagation through biological matter with proper radiation from only two input ports. EMPro simulations were then consulted to determine the behavior of the antenna when inputs 3 and 4 were the only radiating inputs. Results showed that with this setup, the transmitter would still provide the necessary directionality of the 
output radiation pattern. However, it included the drawback of decreased radiation intensity at the receiving end, which was determined as a reasonable tradeoff for testing.

\subsection{Testing the Signal Receiver}

When testing the signal receiver, the best approach was to test each component separately. This included the receiver antenna and RF detector with characterization methods necessary to each component, such as measuring scattering parameters and comparing measurements to data sheets when available.

\subsubsection{Loop Antenna Test Setup and Results}

Based on the work completed in [27], a receiver coil with $2 \mathrm{~mm}$ diameter windings was used to wirelessly receive power from the slot array antenna. This method was implemented as the initial approach for the design of the receiver antenna, but further research determined that a loop antenna with $2 \mathrm{~mm}$ windings was more desirable. A loop antenna was chosen as the simplest implementation because it did not require special manufacturing or design lead time. It was also found that loop antennas have low radiation resistance as well as high tolerance for impedance mismatches from the transmitter, thus making it ideal for easy implementation with any type of transmitter [46].

When creating a loop antenna, a robust design implements a loop with the perimeter matching a full wavelength of the high-frequency signal in free space. The operating wavelength was previously determined at $18.7 \mathrm{~cm}$ in free space, so a piece of magnet wire was cut to the same length for the loop antenna. Conway Mag 28 magnet wire by Consolidated Electronic Wire and Cable Company was chosen in this implementation since magnetic wire increases the sensitivity of receiving loops. Upon 
implementation, the loop was formed and soldered directly to an edge-mount SMA connector. This would allow for easier testing and a direct connection to the RF detector during system integration. The antenna was then connected to the Anritsu 4622B Vector Network Analyzer and wound with $2 \mathrm{~mm}$ diameter loops until the center frequency was at $1.6 \mathrm{GHz}$ in free space. Figure 50 shows the final loop antenna made with magnet wire that was soldered directly to an SMA connector.

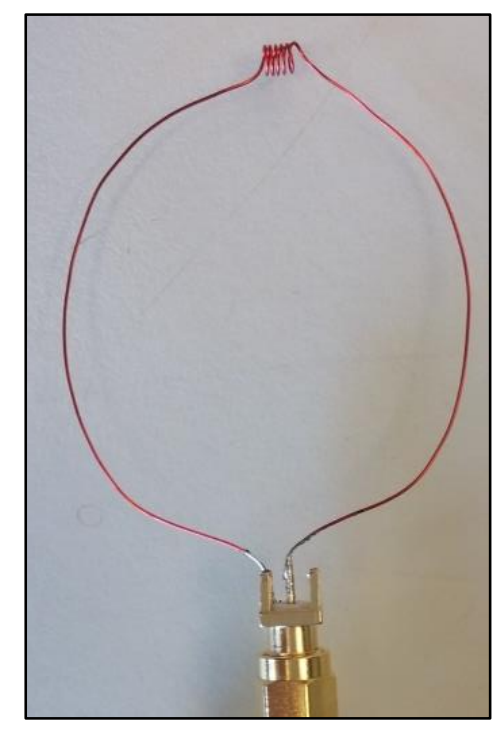

Figure 50: Loop antenna created for receiving the $1.6 \mathrm{GHz}$ transmitted signal.

Measurements made with the Anritsu 4622B Vector Network Analyzer showed $23.5 \mathrm{~dB}$ of loss at $1.6 \mathrm{GHz}$ when the loop antenna was created with 6 windings around $2 \mathrm{~mm}$ of air, as seen in Figure 51. This measurement is desired for similar reasons that a transmitting antenna's scattering parameter at the output requires great loss - for optimal energy capture and the least reflection. This antenna was deemed appropriate for the required use in this glucose sensor and maintained for future testing. 


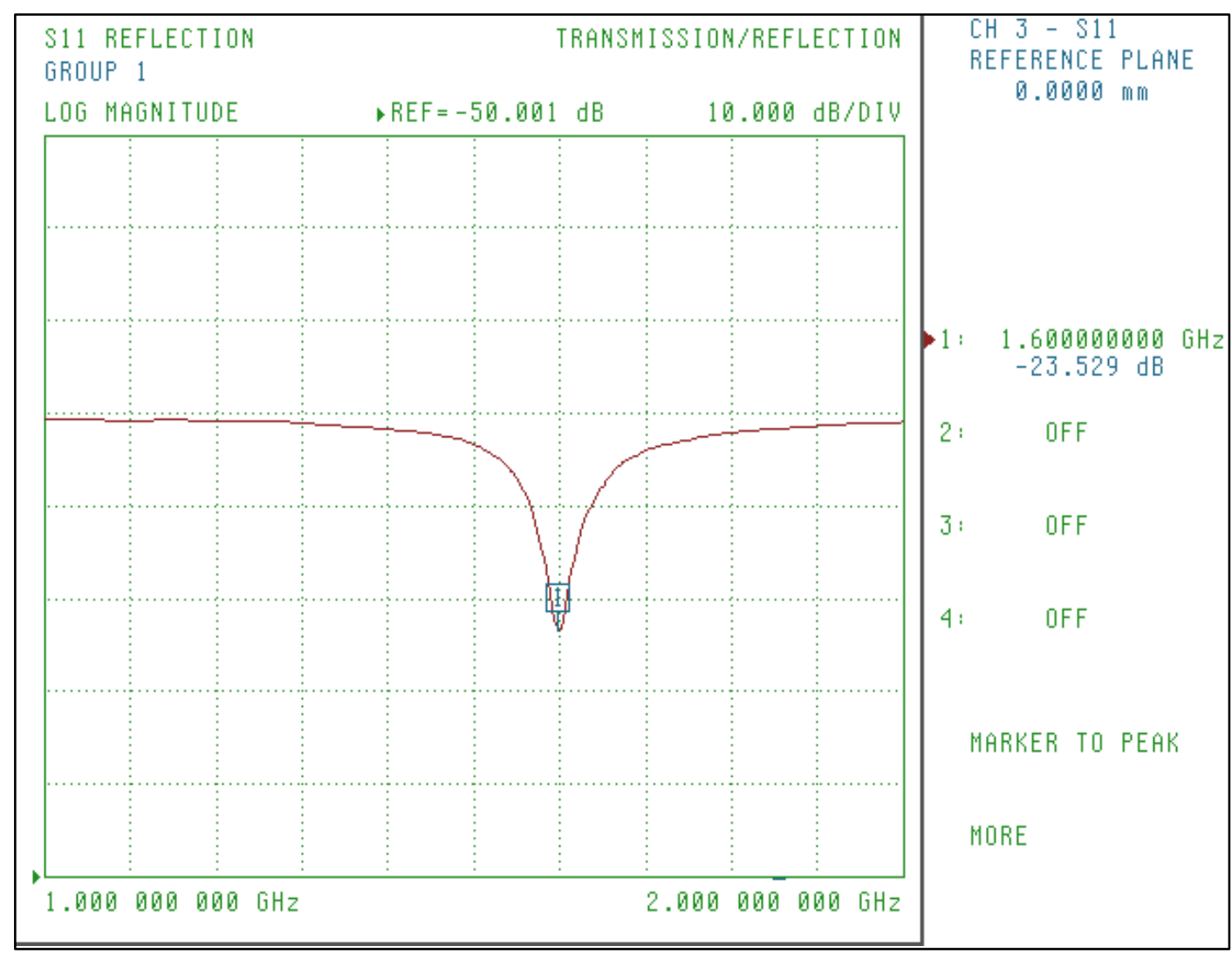

Figure 51: The loop antenna's response at $1.6 \mathrm{GHz}$ showed the desired resonance with $23.5 \mathrm{~dB}$ of loss.

\subsubsection{RF Detector Test Setup and Results}

The most direct method for testing the RF detector was to complete a wired test with known signal dampening connectors. Figure 52 shows the demonstration board implementing the RF detector device by Linear Technology and the DC voltage output connected to the green wire. 


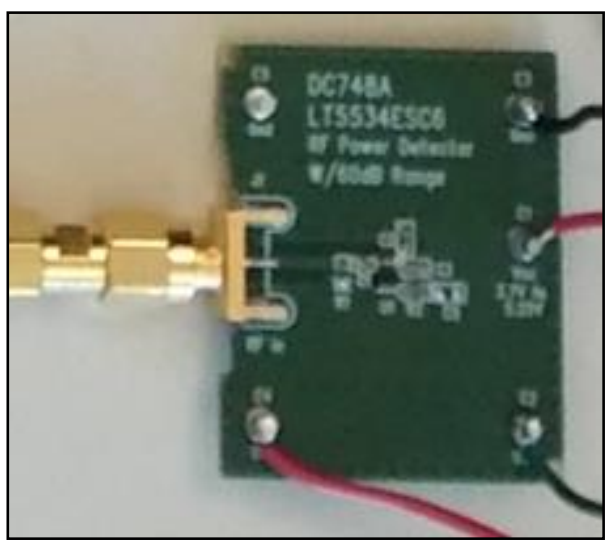

Figure 52: The RF detector demo board with LT5534 detector chip by Linear Technologies was implemented for its broad detection range.

When testing, the $1.6 \mathrm{GHz}$ signal generated after the bandpass filter was directly wired to the input of the RF detector. This measurement was recorded as the maximum signal strength received at $-8.14 \mathrm{dBm}$ and corresponded to a DC voltage output near $2 \mathrm{~V}$. Signal-attenuating connectors were added between the bandpass filter's output and RF detector's input to vary the levels of received power. Five different signal-attenuating connectors were tested, four of which were $-10 \mathrm{~dB}$ and one of which was $-5 \mathrm{~dB}$, to measure five additional levels of received power. Figure 53 displays the recorded values of received power in $\mathrm{dBm}$ and the resulting $\mathrm{DC}$ voltage output. The graph also proves 41 $\mathrm{mV} / \mathrm{dBm}$ slope, as stated by the data sheet [42]. 


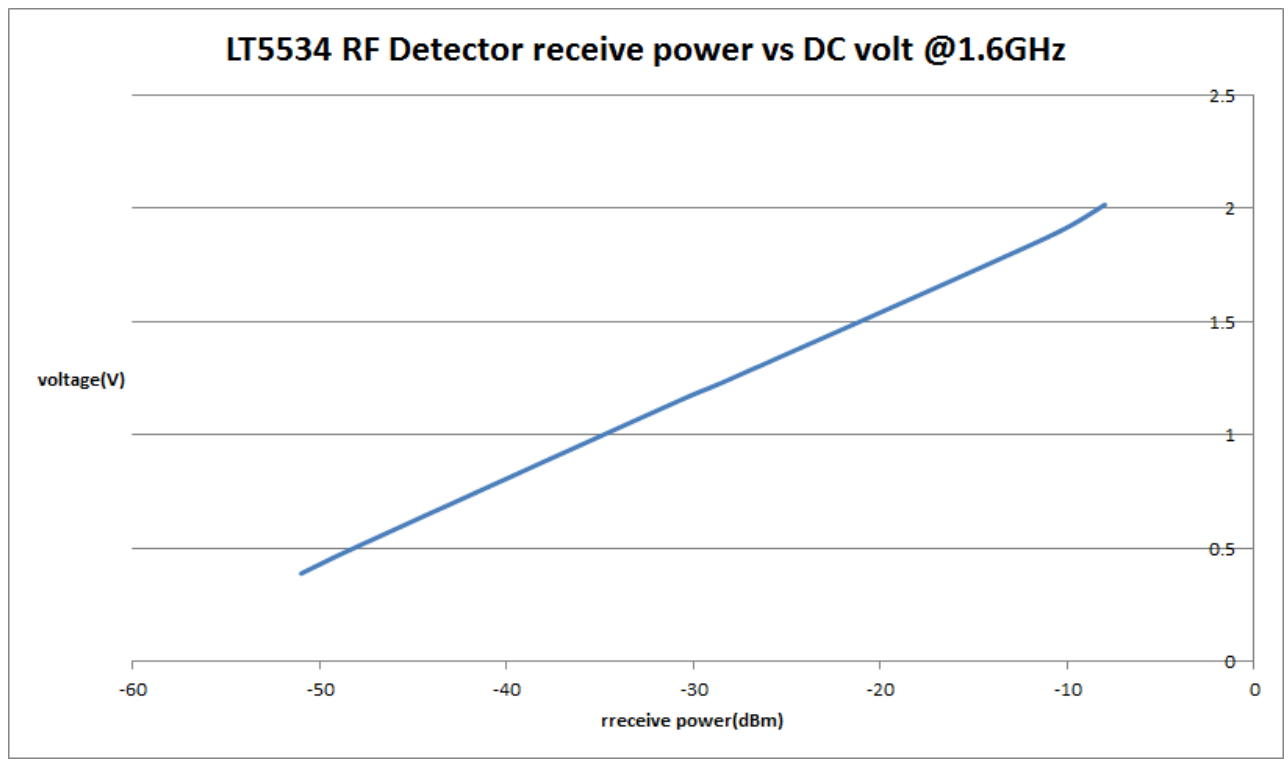

Figure 53: The RF detector's received power and DC voltage output linearly correlated by $41 \mathrm{mV} / \mathrm{dB}[37]$.

\subsection{System Test Setup and Results}

With the component testing complete, the system was ready for setup with various test samples between the transmitting slot array antenna and loop antenna receiver. Measurements were first recorded of the signal strength received after propagating through free space. For this test, the transmitting antenna was taped to the table with the microstrip side down and slot layer face up. This was done to prevent signal reflections propagating from out the microstrip side and around the transmitter to the receiver in hopes of isolating the radiation from the slot side. The receiver was positioned with the loop coils centered above slot 1 with its connector directed from the right, as shown in Figure 54. 


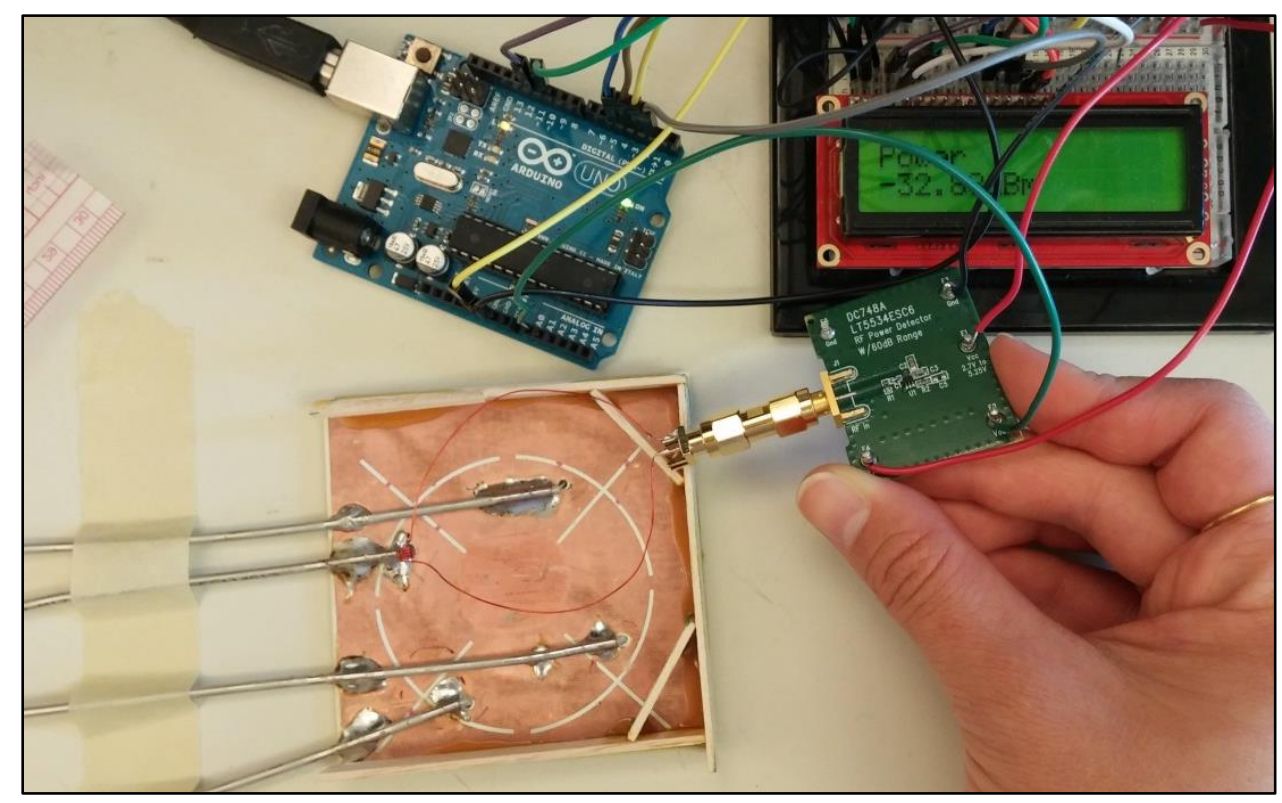

Figure 54: RSSI measurements were taken in free space with the receiver coil centered above input 1 on the slot array transmitter.

Received signal strength measurements were taken while moving the receiver away from the transmitter in increments of $1 \mathrm{~cm}$. The receiver was held parallel to the plane of the antenna transmitter for the duration of the measurements, as shown in Figure 55. The change in received power displayed the expected behavior seen in simulation since the transmitted signal became weaker as it propagated through greater distances to reach the receiver. Figure 56 shows the inverse relationship between received signal strength and distance from the transmitter to receiver. 


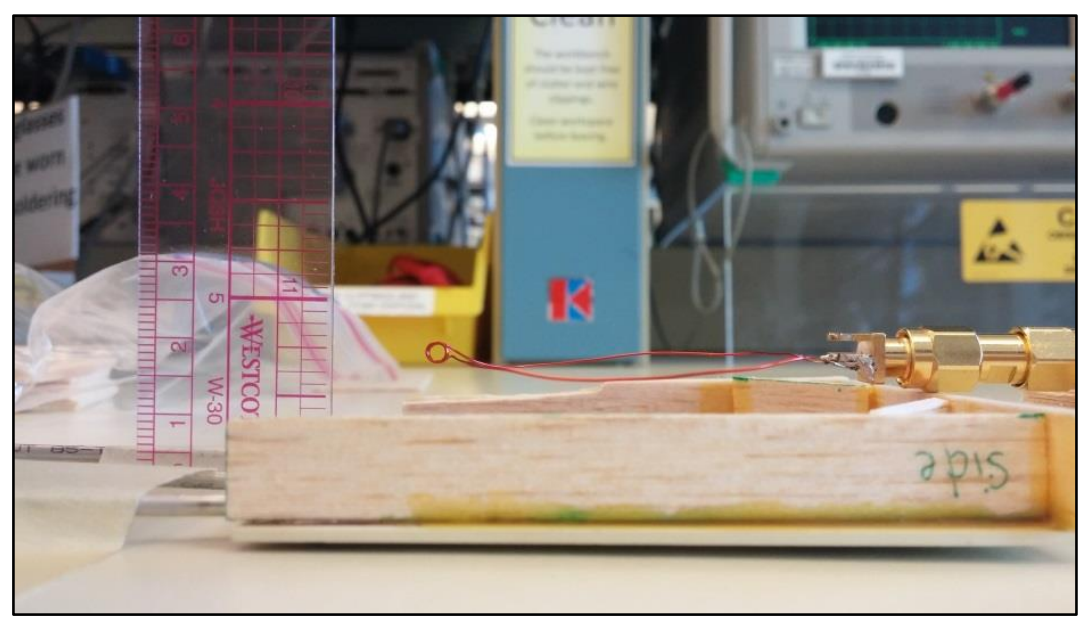

Figure 55: The loop antenna receiver was positioned at varying distances from the transmitter to characterize the system in free space.

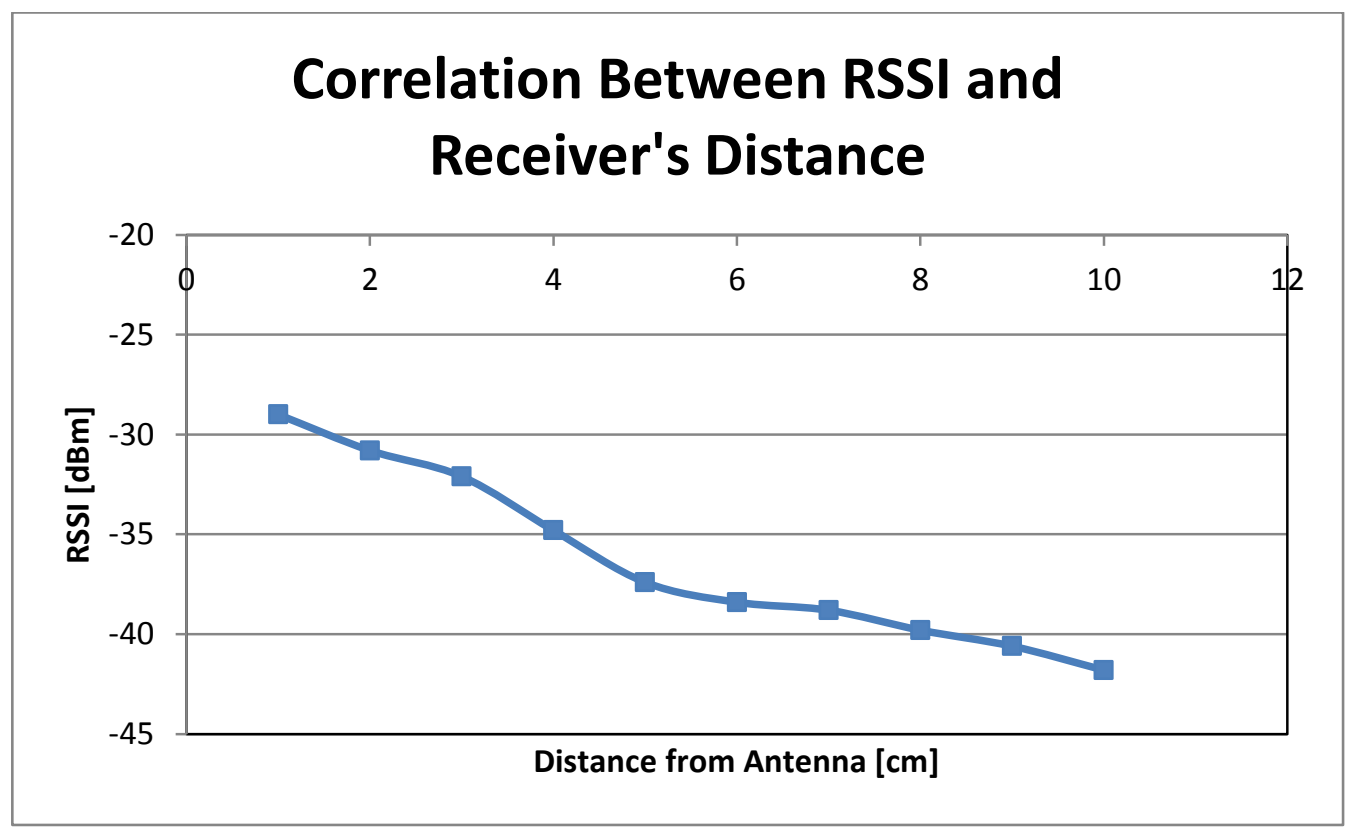

Figure 56: Received signal strength decreased as the receiver moved further away from the antenna.

Upon completion of testing the system in free space, the testing setup was altered to match Figure 57 in which a $1 \mathrm{~cm}$ air gap would need to be maintained during all testing to match simulations. Due to the RF transparent nature of wood, a bolsa wood 
frame was constructed and glued around the edge of the transmitting antenna to provide a $1 \mathrm{~cm}$ buffer between the plane of the antenna and any test samples, as shown in Figure 58. This ensured the required air gap for optimal field propagation as anticipated by the 3-D simulations.

\begin{tabular}{|l|l|}
\hline Receive Loop Antenna & $\rightarrow$ RF Detector $\rightarrow$ Arduino Uno Processor $\rightarrow$ LCD \\
RF Mixer $\rightarrow$ Bandpass Filter $\rightarrow$ Power Divider $\rightarrow$ Slot Array Transmit Antenna \\
\hline
\end{tabular}

Figure 57: Full system diagram with test sample placement specified between the transmitter and receiver.

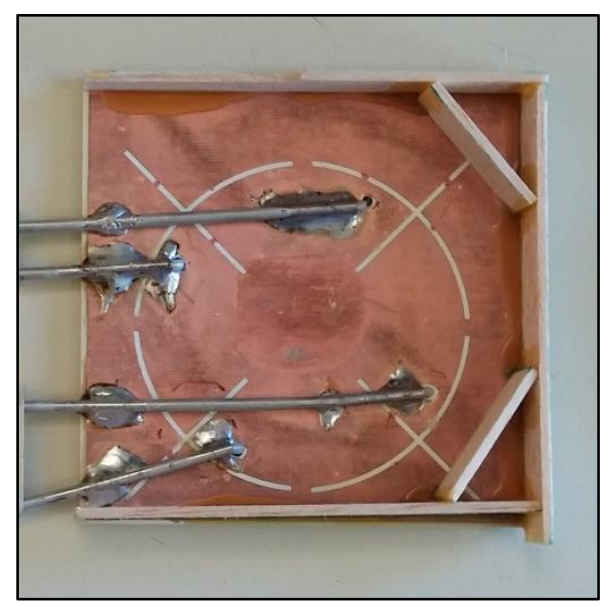

Figure 58: A wooden frame was constructed out of balsa wood to maintain the necessary $1 \mathrm{~cm}$ air gap during testing.

Before testing on any type of sample, the location with the maximum signal strength was determined by taking multiple measurements with the loop antenna receiver at various locations. The receiving antenna was held at a fixed distance from the transmitter while the lateral placement was changed around the slot array antenna. Table 12 lists the received signal strengths at multiple locations of the loop antenna. Based on these measurements, the optimal location of the coil receiver was chosen at slot 2 , 
corresponding to input 2 of the transmitting antenna, with the receiver coils centered directly above the input feed. This location was then used for all measurements with the glucose sensor.

Table 12: Receiver placement around the slot array antenna used to determine the optimal location with maximum received signal strength intensity for future measurements.

\begin{tabular}{|c|c|c|}
\hline Slot Number & Lateral Position & RSSI [dBm] \\
\hline Array & Loop Centered on Array & -30.1 \\
\hline \multirow{3}{*}{ Slot 1} & Coil Centered on Slot & -24.1 \\
\hline \multirow{3}{*}{ Slot 2} & Loop Centered on Slot & -30.9 \\
\hline \multirow{4}{*}{ Slot 3} & Coil at Input of Slot & -31.3 \\
\hline \multirow{4}{*}{} & Coil Centered on Slot & -19.2 \\
\hline & Loop Centered on Slot & -19.3 \\
\hline \multirow{3}{*}{ Slot 4} & Coil at Input of Slot & -17.5 \\
\hline & Coil Centered on Slot & -22.4 \\
\hline & Loop Centered on Slot & -29.9 \\
\hline & Coil at Input of Slot & -28.9 \\
\hline & Coil Centered on Slot & -26.2 \\
\cline { 2 - 3 } & Loop Centered on Slot & -24 \\
\hline & Coil at Input of Slot & -21.1 \\
\hline
\end{tabular}

\subsubsection{System Test on Blood Mimicking Phantoms}

Completing testing on controlled samples with blood glucose levels was pertinent to characterizing the accuracy of the sensor. A study in which tissue mimicking "phantoms" were developed for the identical purpose of testing a noninvasive glucose sensor provided step-by-step instructions on how to construct such phantoms that mimicked the electrical properties of human tissues [47]. The main advantage in using the same phantoms created by Yilmaz, et al. was their use of ingredients that could be found in the average grocery store, such as vegetable oil, gelatin, and liquid dish detergent. Additionally, their phantoms were more solid and rigid than the typical 
phantom gels created by other scholarly groups, which aided in maintaining a consistently physical form across all test samples.

The testing completed by Yilmaz, et al. proved that their phantoms closely matched the electrical properties of human fat, blood, muscle, and skin across $200 \mathrm{MHz}-$ $18 \mathrm{GHz}$. To verify the accuracy of their phantoms, Yilmaz, et al. measured the electrical properties of their phantoms with the Agilent 85070E Dielectric Probe kit across the specified frequency range. The group then drew comparisons to other phantoms created and presented in scholarly articles, which closely matched their measured values. For the purpose of testing this glucose sensor, a similar procedure was completed to make blood samples of varying glucose levels with the detailed setup listed in Appendix I.

The blood phantoms were created in the microfabrication clean room on the Cal Poly campus due to the availability of necessary equipment, including scales, beakers, and hot plates. During the procedure of making the phantoms, some changes to the original instructions were implemented to ensure homogenous mixing of all blood phantoms. This was determined as an acceptable change since the homogeneity of the blood phantoms was more significant than the potential drawbacks of deviating from the specified instructions. Seven different blood phantoms were made with this changed procedure, each with different blood glucose levels.

To determine the range of the sensor, a blood phantom without any added glucose was tested against a phantom with a large amount of glucose. The phantom with added glucose corresponded to a glucose concentration of $2505 \mathrm{mg} / \mathrm{dL}$, far beyond any average blood glucose level. This was merely used as a comparison in the received signal strength of two extremely different blood glucose levels. The measurement was 
completed 16 hours after fabricating the phantom, and the phantom was stored at room temperature during that time. Figure 59 shows the two phantoms created for this test, each of which measured $2.5 \mathrm{~cm}$ in thickness.

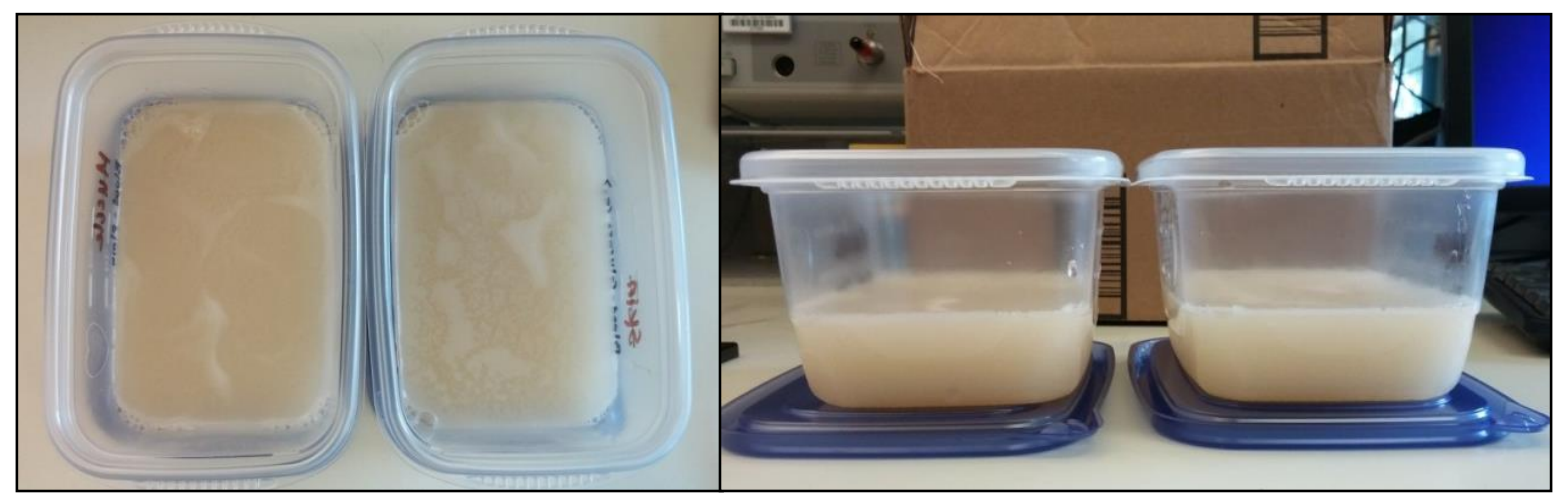

Figure 59: Top view and side view of the two fabricated blood phantoms. One phantom contained no added glucose (left) while the other contained 5 grams of added glucose (right).

Figure 60 also shows the test setup with the blood phantoms. The entire blood phantom was placed on a piece of balsa wood resting on the frame of the transmitting antenna to hold the blood phantom at a level surface. The loop antenna was supported by pieces of balsa wood to keep the structure and shape of the loop receiver throughout the testing procedure. This support also prevented direct contact of the receiving antenna with the sample since direct contact could alter the received signal. Measurements were taken to compare the received signal strength of the antenna with and without the balsa wood to ensure that the wood did not interfere with the RSSI. At $2.25 \mathrm{~cm}$ above the plane of the antenna, the loop antenna consistently received $-29.1 \mathrm{dBm}$ with and without the balsa wood support. The RF detector board was also set on top of a makeshift stand with pieces of wood used to stabilize the device. 


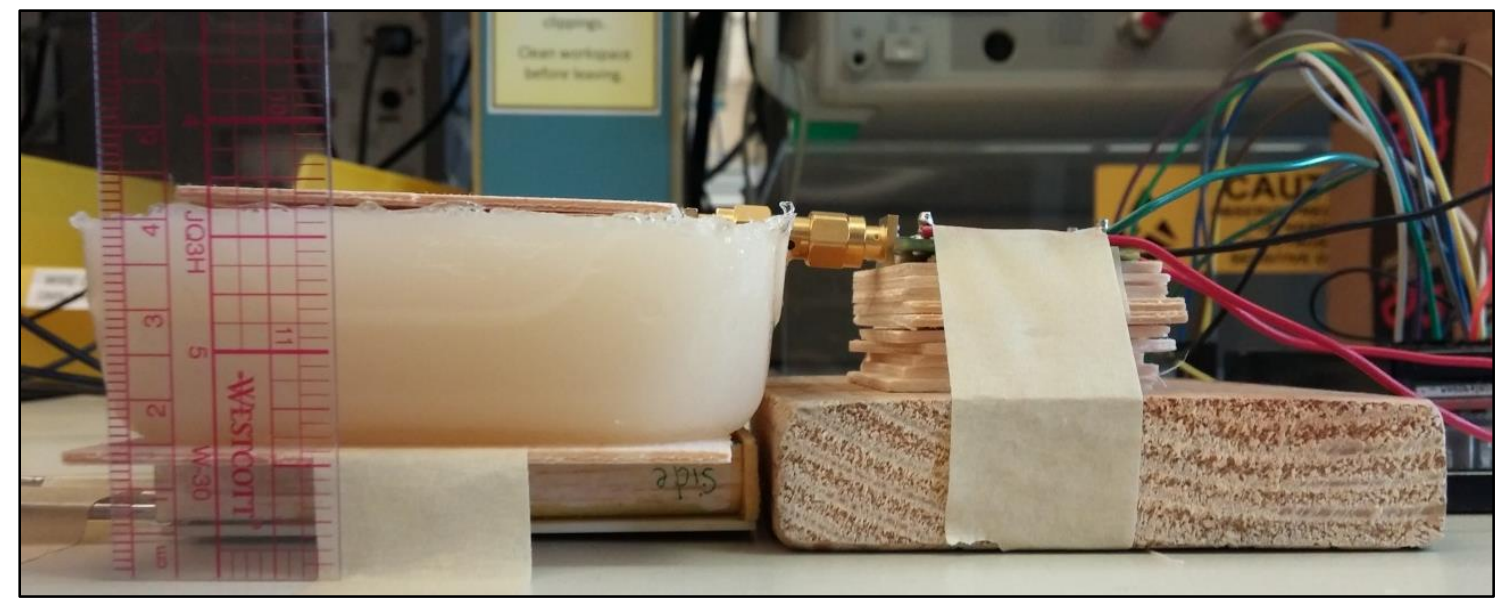

Figure 60: The test setup for the initial blood glucose phantoms placed the entire blood phantom on top of the transmitting antenna with the receiver placed directly on the phantom.

Measurements with the receiver at the same location displayed a slight change in RSSI based on the variation in electrical properties of the blood phantom. With the receiver fixed at a height of $4 \mathrm{~cm}$ above the plane of the antenna, the RSSI was recorded for no sample between the transmitter and receiver, for the blood with no glucose, and for the blood saturated with glucose. The measurement through air with no blood phantom was $-39.09 \mathrm{dBm}$, and the received signal strengths decreased as expected with the two different blood phantoms. The unsaturated blood phantom measured $-48.6 \mathrm{dBm}$, while the glucose-saturated blood phantom measured $-49.16 \mathrm{dBm}$. Although not a significant difference between the two measurements, the variation in RSSI demonstrated a change in electrical properties of the sample being measured. This concept was further tested with five additional blood phantoms each at different glucose concentrations. Each blood phantom was first sliced into $6 \mathrm{~mm}$ thick pieces with a traditional cheese slicer to decrease the sample size and hopefully increase the sensitivity of the loop antenna 
receiver. The receiver was fixed at a height $1.5 \mathrm{~cm}$ above the plane of the antenna, and the results are shown in Appendix II.

The measurements were expected to decrease in signal strength as the glucose concentration increased for the blood phantoms. This was not the case with these measurements, and could be attributed to a number of causes. First, the most probable cause of unexpected behavior was the change in fabrication procedure for the blood phantoms. By changing the procedure, changes to the chemical composition of the blood phantoms could have occurred and changed the electrical characteristics from those produced by Yilmaz, et al. An additional cause for a change in behavior was the deviation in phantom thickness. Although a cheese slicer was used to create uniform thicknesses of the blood phantoms, some deviations in cut thickness occurred. This potentially altered the path of the transmitted signal by lengthening or shortening the propagation path to the receiver. Further tests were completed on human subjects to determine the feasibility of the glucose sensor since the tests on blood phantoms were not completely conclusive.

\subsubsection{System Test on Human Subjects}

The noninvasive glucose sensor was tested on a total of eight human subjects, all of varying gender, age, and size. Each individual followed a process of measuring their blood glucose from a physical blood sample with the Kroger WaveSense Glucometer. This was followed by an RSSI measurement from the developed glucose sensor. The transmitting antenna was placed on the inner side of the subject's biceps brachii on the right arm, while the receiving antenna was placed on the outer side of the arm. An elastic band was used to tie both the transmitter and the receiver to the biceps brachii, as shown 
in Figure 61. The direction of the transmitter and receiver were maintained for each measurement on human subjects.

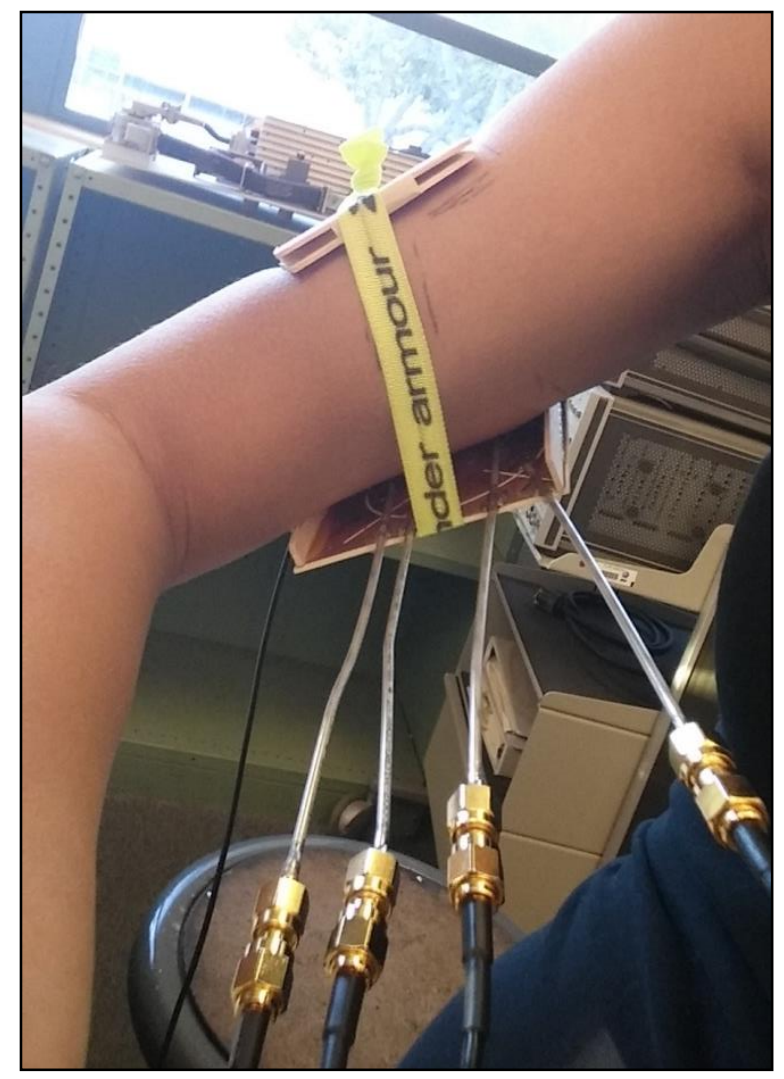

Figure 61: The test setup on human subjects involved tying the transmitter and receiver to the biceps with an elastic band.

The first human subject test was completed in a single day from 10:00am to 4:30pm with the same test subject. During this time, ten measurements of blood glucose and RSSI were recorded while the subject consumed food to vary the blood glucose levels. The placement of the transmitter and receiver were carefully maintained by physically marking the outline of the components on the subject's arm.

For the first four measurements, the receiving antenna was directed with the coil loops in the clockwise direction, while the six remaining measurements were recorded with the coil loops in the counter-clockwise direction. Table 13 shows the recorded 
values from these tests, and Figure 62 and Figure 63 plot these values. The graphs indicate that the sensed RSSI correlated to the blood glucose levels as expected, with the exception of two outlier data points. The data trends from the two graphs indicate a nonlinear relationship between the measured blood glucose levels and RSSI. However, a linear trend line was established to model the inverse relationship between RSSI and blood glucose levels. The inclusion of all data points in Figure 63 demonstrates the inverse relationship but with a smaller coefficient of determination, so the trendline shown excludes the greatest outlier data point to achieve a more accurate coefficient of determination.

Table 13: Measured blood glucose and RSSI were recorded for the first human subject with two orientations of the receiving antenna.

\begin{tabular}{|l|c|c|c|c|}
\hline & Test & Time & $\begin{array}{c}\text { Measured Blood } \\
\text { Glucose [mg/dL] }\end{array}$ & $\begin{array}{c}\text { RSSI } \\
{[\mathbf{d B m}]}\end{array}$ \\
\hline & 1 & $10: 10$ & 87 & -50 \\
\hline Clockwise & 2 & $11: 15$ & 142 & -57 \\
\hline Direction & 3 & $12: 05$ & 126 & -56.7 \\
\hline & 4 & $12: 35$ & 115 & -55.2 \\
\hline & 5 & $13: 00$ & 92 & -54.9 \\
\hline Counter- & 6 & $14: 00$ & 83 & -54.4 \\
\hline Clockwise & 7 & $14: 15$ & 91 & -55.1 \\
\hline Direction & 8 & $15: 00$ & 159 & -57.7 \\
& 9 & $16: 00$ & 148 & -56.7 \\
\hline & 10 & $16: 30$ & 83 & -55.9 \\
\hline
\end{tabular}




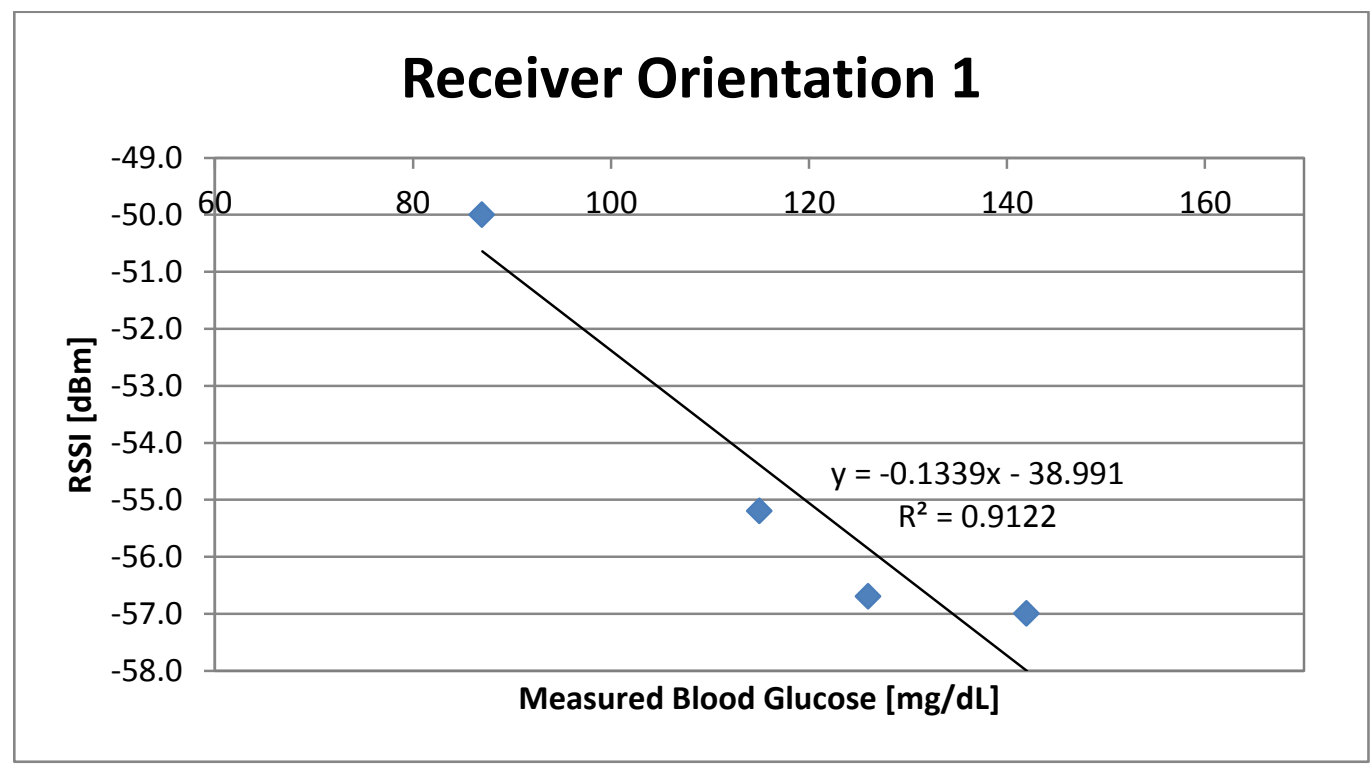

Figure 62: Measurements from the receiving antenna in the first orientation showed the expected inverse relationship between glucose and RSSI.

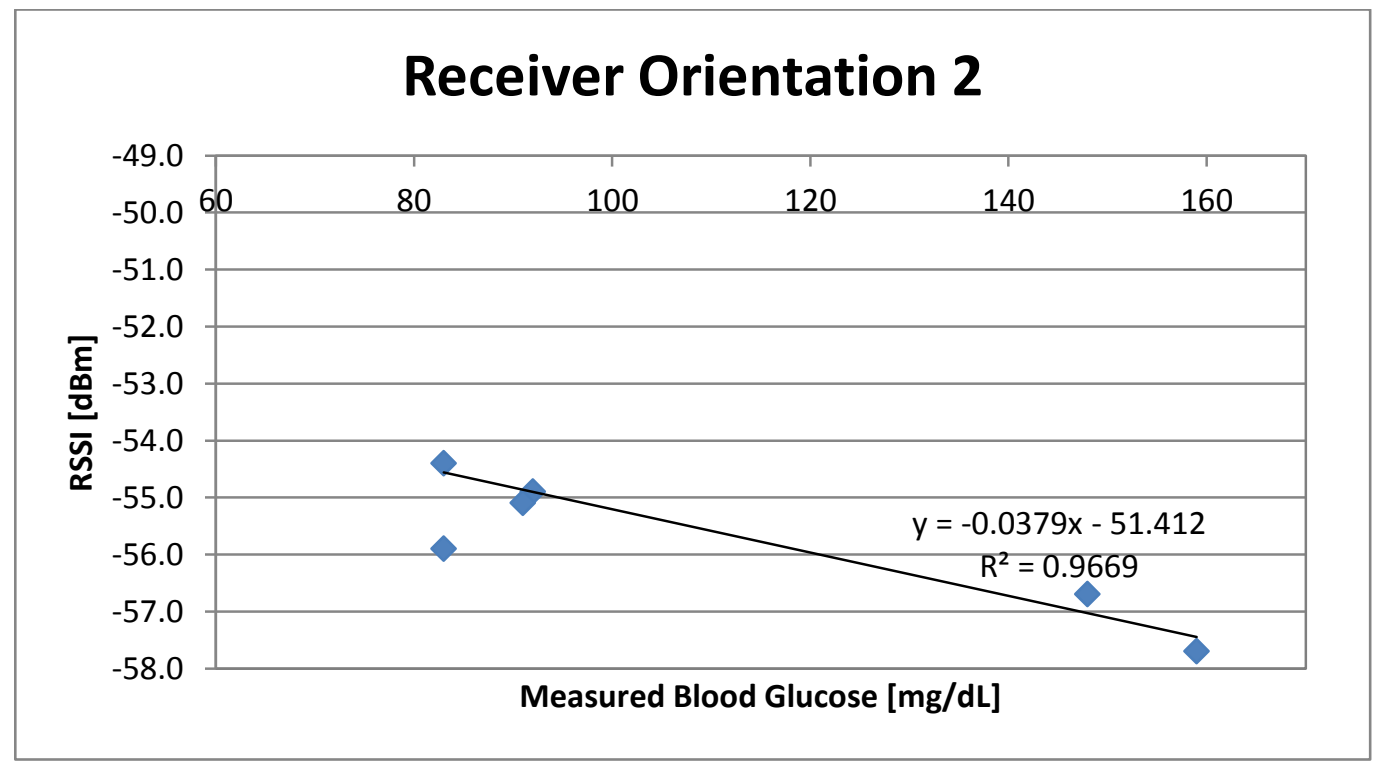

Figure 63: Measurements from the receiving antenna in the second orientation showed the expected inverse relationship between glucose and RSSI with two outliers.

These promising results were an indication that the glucose sensing system accurately tracked changes in glucose but required verification through additional testing on more subjects. It was taken into account that since the anatomy of each subject would 
vary, the specific rate of change from RSSI to measured blood glucose level would not match across each subject. The only expected similarity would be the inverse relationship between the two measurements as an indicator of accurately tracking changes in glucose.

Seven more test subjects volunteered to participate in the research, and each additional subject varied in age, gender, and physical size. Two of these subjects had been previously diagnosed with diabetes, while the remaining five had not. Each test subject was requested to measure their blood glucose levels with the Kroger WaveSense Glucometer and the designed glucose sensor at least twice with an hour of separation between measurements. They were also requested to measure the fat percentage of their arm with the Tanita BC-601FS FitScan segmental body composition monitor, as well as the circumference of their upper arm while relaxed and flexed. These anatomical measurements were used to determine a ratio of muscle-to-fat as an indicator of variation between each subject. Some arm fat percentage measurements of the same subject varied per test, so these measurements were averaged per subject.

An estimate of the cross-sectional area of the biceps brachii was determined by factoring out the ratio of a flexed muscle's length compared to a relaxed muscle's length. Previous studies have shown that when contracting, a muscle's length will shorten to about $57 \%$ of its relaxed length [48]. Therefore, considering a muscle that maintains its volume when contracted and at rest would require an increase in radius by one-third to compensate for this decrease in length. These ratios provided a means of estimating the cross-sectional area of the biceps brachii with the following equation:

$$
\text { radius }_{\text {flexed }}-\text { radius }_{\text {relaxed }}=\frac{4}{3} * \text { radius }_{\text {muscle }}
$$


The equation was used to determine an approximate cross-sectional area of muscle to establish a ratio of muscle-to-fat as a characterization method of the subjects.

Table 14: Characteristics of each test subject led to the estimation of their muscle-to-fat ratio for a cross-section of their upper arm.

\begin{tabular}{|c|c|c|c|c|c|c|c|c|}
\hline & $\begin{array}{c}\text { Subject } \\
\mathbf{A}\end{array}$ & $\begin{array}{c}\text { Subject } \\
\text { B }\end{array}$ & $\underset{\text { C }}{\text { Subject }}$ & $\begin{array}{c}\text { Subject } \\
\text { D }\end{array}$ & $\begin{array}{c}\text { Subject } \\
\mathbf{E}\end{array}$ & $\begin{array}{c}\text { Subject } \\
\text { F }\end{array}$ & $\begin{array}{c}\text { Subject } \\
\text { G }\end{array}$ & $\begin{array}{c}\text { Subject } \\
1\end{array}$ \\
\hline $\begin{array}{c}\text { Circumference } \\
\text { of Relaxed } \\
\text { Biceps }[\mathrm{cm}]\end{array}$ & 34.5 & 24 & 26 & 28.25 & 30 & 23.5 & 28 & 24.25 \\
\hline $\begin{array}{c}\text { Radius of } \\
\text { Relaxed Biceps } \\
{[\mathrm{cm}]}\end{array}$ & 5.49 & 3.82 & 4.14 & 4.50 & 4.77 & 3.74 & 4.46 & 3.86 \\
\hline $\begin{array}{c}\text { Circumference } \\
\text { of Flexed } \\
\text { Biceps [cm] }\end{array}$ & 38.5 & 26.25 & 29.5 & 30 & 32.5 & 24 & 28.5 & 26.50 \\
\hline $\begin{array}{c}\text { Radius of } \\
\text { Flexed Biceps } \\
{[\mathrm{cm}]}\end{array}$ & 6.13 & 4.18 & 4.70 & 4.77 & 5.17 & 3.82 & 4.54 & 4.22 \\
\hline $\begin{array}{c}\text { Estimated } \\
\text { Surface Area of } \\
\text { Biceps Cross- } \\
\text { Section }\left[\mathrm{cm}^{2}\right]\end{array}$ & 2.26 & 0.72 & 1.73 & 0.43 & 0.88 & 0.04 & 0.04 & 0.72 \\
\hline $\begin{array}{c}\text { Average Fat } \\
\text { Percentage of } \\
\text { Arm [\%] }\end{array}$ & 18.85 & 22.93 & 12.10 & 24.90 & 41.95 & 25.15 & 24.60 & 22.40 \\
\hline $\begin{array}{l}\text { Ratio of Muscle } \\
\text { to Fat }\left[\mathrm{cm}^{2} / \%\right]\end{array}$ & 0.120 & 0.031 & 0.143 & 0.017 & 0.021 & 0.001 & 0.001 & 0.03 \\
\hline
\end{tabular}

Measurements were recorded for each subject at various times between meals to create a change in blood glucose levels. The variation in body compositions indicated in Table 14 shows the different anatomies of each test subject, and Table 15 provides the measured results. While taking these measurements, it is important to note that subjects $\mathrm{B}, \mathrm{E}$, and $\mathrm{G}$ wore sleeved clothing that prohibited the components from directly touching their skin. It was established that the clothing was thin and made of natural, nonconductive fabric that would not impede the transmitted or received signal. 
Table 15: Each test subject underwent two or three tests to determine a trend in measurements.

\begin{tabular}{|c|c|c|c|c|c|c|c|c|}
\hline & & $\begin{array}{c}\text { Subject } \\
\text { A }\end{array}$ & $\begin{array}{c}\text { Subject } \\
\text { B }\end{array}$ & $\begin{array}{c}\text { Subject } \\
\text { C }\end{array}$ & $\begin{array}{c}\text { Subject } \\
\text { D }\end{array}$ & $\begin{array}{c}\text { Subject } \\
\text { E }\end{array}$ & $\begin{array}{c}\text { Subject } \\
\text { F }\end{array}$ & $\begin{array}{c}\text { Subject } \\
\text { G }\end{array}$ \\
\hline \multirow[t]{2}{*}{ Measurement 1} & $\begin{array}{l}\text { Blood } \\
\text { Glucose } \\
\text { Level } \\
{[\mathrm{mg} / \mathrm{dL}]}\end{array}$ & 139 & 94 & 94 & 95 & 105 & 87 & 153 \\
\hline & $\begin{array}{c}\text { RSSI } \\
{[\mathrm{dBm}]}\end{array}$ & -50.3 & -46.5 & -46.6 & -48.5 & -52.5 & -48.6 & -53.2 \\
\hline \multirow[t]{2}{*}{ Measurement 2} & $\begin{array}{c}\text { Blood } \\
\text { Glucose } \\
\text { Level } \\
{[\mathrm{mg} / \mathrm{dL}]}\end{array}$ & 109 & 95 & 115 & 103 & 107 & 151 & 77 \\
\hline & $\begin{array}{c}\text { RSSI } \\
{[\mathrm{dBm}]}\end{array}$ & -48.9 & -44.7 & -53.3 & -52.3 & -52.1 & -48.7 & -52.9 \\
\hline \multirow[t]{2}{*}{ Measurement 3} & $\begin{array}{c}\text { Blood } \\
\text { Glucose } \\
\text { Level } \\
{[\mathrm{mg} / \mathrm{dL}]}\end{array}$ & N/A & 124 & 84 & 86 & N/A & N/A & 154 \\
\hline & $\begin{array}{c}\text { RSSI } \\
{[\mathrm{dBm}]}\end{array}$ & N/A & -49.03 & -52.1 & -55.1 & N/A & N/A & -53.4 \\
\hline
\end{tabular}

Figure 64: The measured RSSI and blood glucose levels varied across each test subject, yet 5 of the subjects' data reveals the expected inverse relationship between the two values. indicates that the expected behavior between RSSI and measured blood glucose was accurately sensed for five of the seven subjects when purely analyzing the linear trend lines. Although the linear trend lines include the numerous outliers, these data points still exist and should be taken into consideration. 


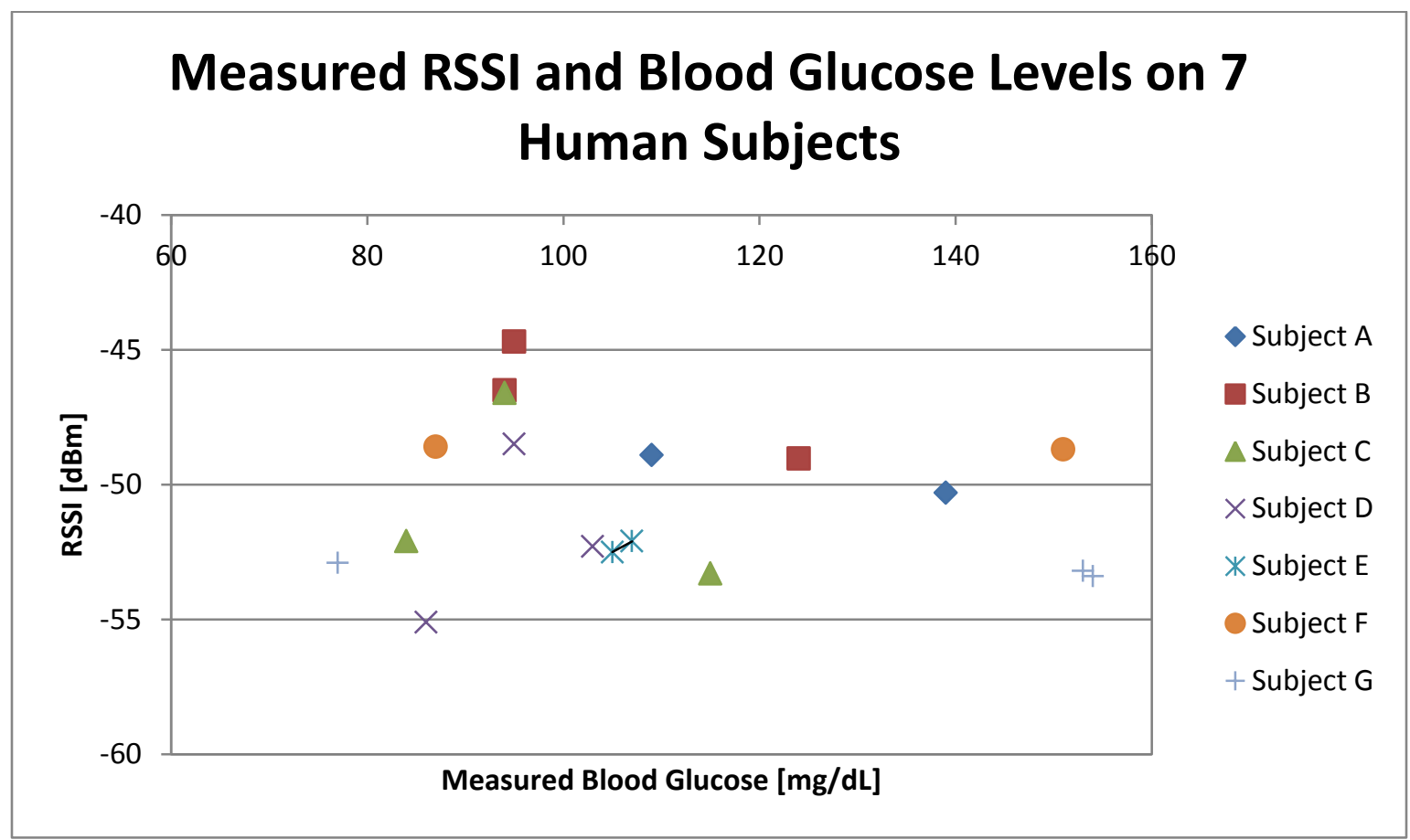

Figure 64: The measured RSSI and blood glucose levels varied across each test subject, yet 5 of the subjects' data reveals the expected inverse relationship between the two values.

All but one test subject had some outlier that did not match the expected behavior of the other obtained data points. This exception was Subject A, who also had the largest circumference of the upper arm and the greatest estimated cross-sectional area of muscle. This factor could have contributed to more accurate measurements since the receiver was able to lay completely flush against the surface of the subject's arm, allowing for more stability in the receiver's measurements. Subjects B and C also showed a close relation to the expected results with the exception of one data point. Their results were analyzed with a linear trend line that reflects the inverse relationship between blood glucose and received signal strength, as seen in Figure 65. The outlier was probably attributed to the smaller circumferences of the subjects' arms which prevented the receiver from lying 
completely flush against the outer biceps. Any distance between the receiver and the arm would make the receiving loop more susceptible to noise in the form of signal reflections from the transmitter. This situation is displayed in Figure 66 in which an air gap is created when the receiver does not lay completely flush against the outer biceps.

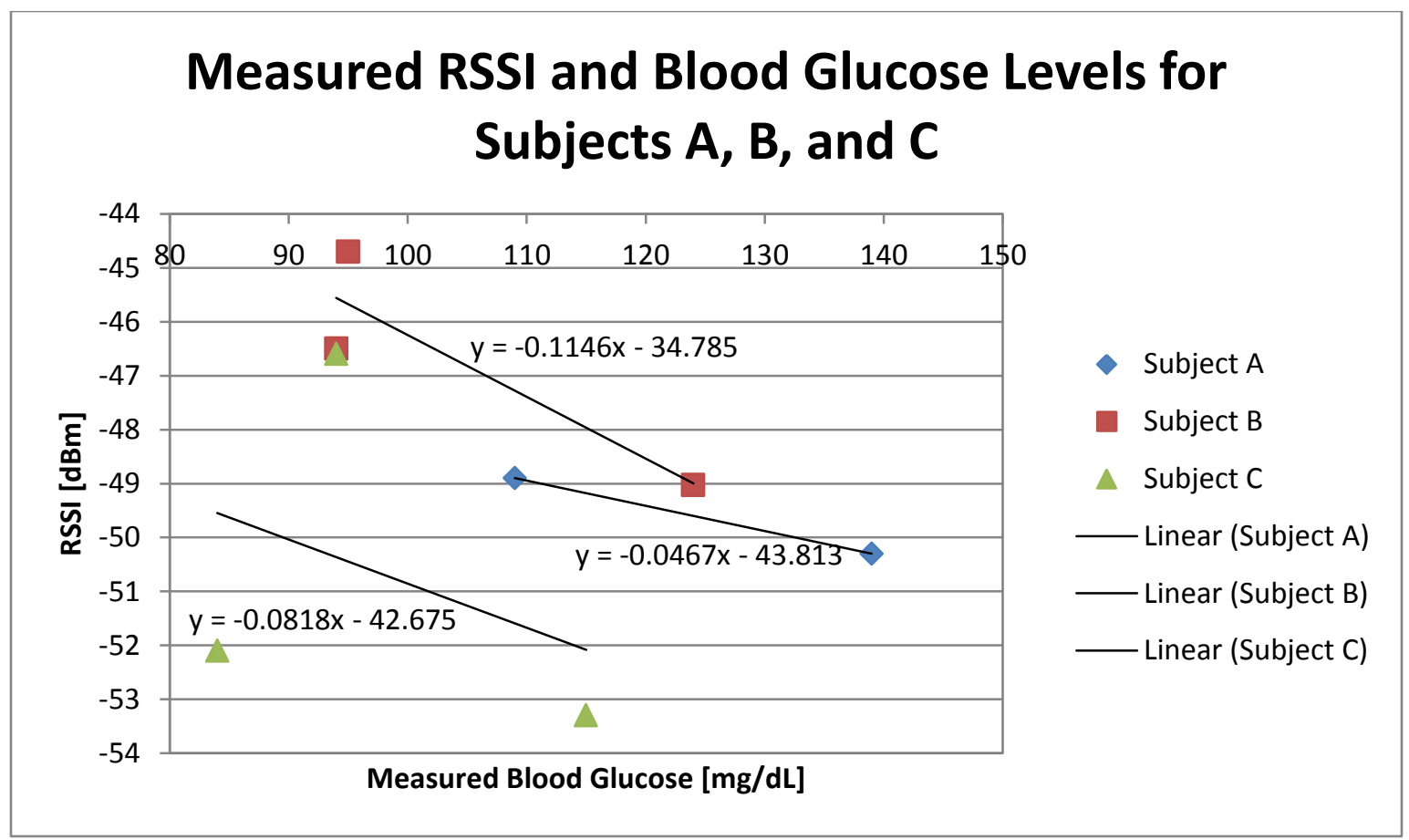

Figure 65: With the exception of a few data points, the sensed and measured blood glucose levels for Subjects A, B, and C display a trend similar to the expected relation. 


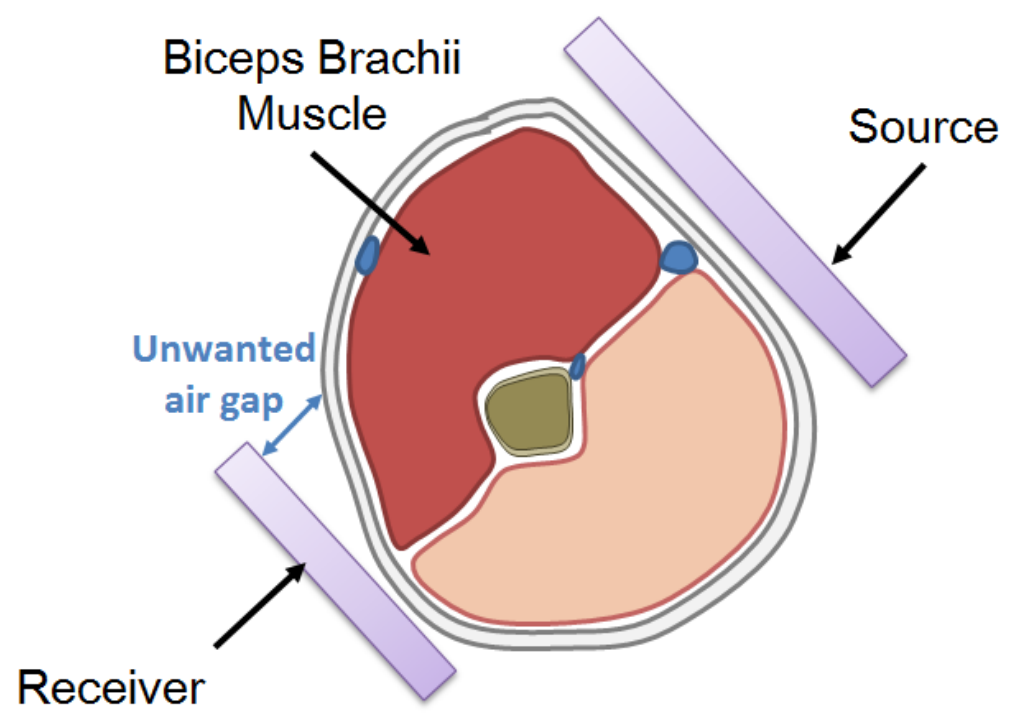

Figure 66: Subjects with smaller arm circumferences were found to have varying measurements attributed to distance between the receiver and arm. 


\section{Chapter 5: Conclusion and Future Considerations}

\subsection{Summary and Conclusion}

The work completed in this thesis was an investigation into a new, noninvasive sensing method for blood glucose levels. This new design eliminated any need for obtrusively obtaining samples from the body and for chemical analysis with costly test strips by purely focusing on the characteristics of a received RF signal in the lowgigahertz range. The power of the received signal acted as an indicator of blood glucose levels since research has proven the effect of changing glucose on electrical properties of blood. These studies have shown that increasing blood glucose levels decrease the dielectric permittivity of blood, therefore acting as a medium with weaker energy storage capabilities and weaker capacitance. This decreased capacitance increases the medium's impedance at high frequencies, thus decreasing the energy and propagation distance of a transmitted signal through the medium. The expected behavior modeled an inverse relation between blood glucose levels and received signal strength.

A system of components was designed to support a slot array antenna transmitter and loop antenna receiver based on a design for wireless power transmission to implanted receivers [27]. The optimal operating frequency was researched through EMPro simulations exhibiting that maximum energy absorption for glucose occurred at $1.6 \mathrm{GHz}$. The components included an RF mixer, bandpass filter, and Wilkinson power splitter for the signal transmission, which were necessary to implement the system in the Microwave Engineering lab at Cal Poly San Luis Obispo. Each component associated with the signal transmission was designed for an operational frequency of $1.6 \mathrm{GHz}$ and created on campus. 
A loop antenna receiver was created from magnet wire to receive signals at 1.6 $\mathrm{GHz}$ for the sensor. The loop antenna was connected to an RF detector and Arduino Uno, which were implemented to receive the transmitted signal at intensities as low as $60 \mathrm{dBm}$. This range was necessary as the transmitted signal was found to be near $-35 \mathrm{~dB}$ per input and was expected to decrease after travelling through biological material. The Arduino Uno was used to directly read the received power without any additional calculations or processing necessary.

The system was tested on phantoms of blood infused with glucose to mimic samples with various levels of glucose concentrations. Based on two tests between a sample heavily infused with glucose and a sample without glucose, a correlation between glucose levels and received power was made. However, further testing on five samples with realistic blood glucose values was inconclusive for multiple reasons including fabrication error.

Additional testing on eight human subjects revealed a consistent trend in tracking blood glucose changes when set up properly. The accuracy of this tracking severely depends on the precise placement consistency with the transmitting antenna and receiving antenna, which was demonstrated by the results from the day-long 10 measurement tests versus the half-day 3 measurement tests. The day-long test completed on a single subject with precise placement control produced the most valuable results, which verified the system's capability of non-invasively tracking changes in glucose levels.

Almost all data on human subjects shows the inverse relationship between blood glucose levels and received power with a decreasing linear trend line. These trend lines demonstrate the predicted concept for tracking blood glucose while maintaining 
consistent sensor placement. Expectations of varying accuracy based on body composition were met when analyzing data for eight subjects, each with different upper

arm muscle-to-fat ratios. Results determined that a larger circumference of the upper arm typically indicated greater muscle mass and produced more accurate results. Noise interference did degrade the stability and accuracy of the remaining test subjects; however, the generic trend found through the research results indicated that the method adequately tracked changes in blood glucose levels.

\subsection{Future Considerations}

Upon improving this noninvasive glucose sensing design, two key figures of merit hold greatest priority - accuracy and portability. For the ultimate goal of reaching the consumer market, the system must withhold a high level of consistency and accuracy with every measurement. This would entail improvements on the structure of the loop antenna receiver to maintain stable measurements. The current design with the magnet wire loop antenna affixed to wood lacked flexibility to properly fit each user, as indicated by the unstable results. An optimal form for the receiving loop antenna would be a flexible printed circuit or "tattoo" style circuit for eliminating obtrusiveness and the chance for noise interference. RF-blocking foam covering the outer side of the receiver would also increase the receiver's accuracy from person to person since it would isolate and measure solely the signals propagating through the biceps. Additional signal processing to average the received signal intensities over a short time span would further enhance the accuracy of the measurements. This could be done with the Arduino Uno development board currently in use for displaying the received power measurements. 
A key factor in obtaining high accuracy is determining a calibration method for the system. Since each user has a unique anatomy and biological volume of fat, muscle, and blood, a reliable calibration sequence to determine the true values of blood glucose levels instead of a relative measurement would be necessary to bring the sensor to market. A calibration technique could involve some sort of sequence in which the user measures the fat percentage of their arm and factors that measurement into their prebreakfast blood test to set a baseline value. Creating this baseline would provide a stable means of accurately reading future measurements throughout the day. The calibration technique could be further enhanced with a method of determining skin moisture, which could also alter the received signal strength. A signal transducer could potentially fulfill this function by transmitting a short pulse and determining the reflection off the skin as an artificial noise floor. This calculated noise floor could then be factored out of the received signal strength to determine the true intensity of the received signal.

Changing the receiver's form factor in the described methods would also improve the portability of the device to enhance the consumer marketability of the system. Additionally, the portability could be expanded upon with a custom circuit board for the signal generation. A PCB that produces four individual $1.6 \mathrm{GHz}$ output signals would untether the transmitting antenna from the lab bench. This would overall promote the current design from prototype to consumer-level product. 


\section{REFERENCES}

[1] Center for Disease Control, "Infographic: A Snapshot of Diabetes in America," 11 June 2014. [Online]. Available: http://www.diabetes.org/diabetesbasics/statistics/cdc-infographic.html. [Accessed 15 October 2014].

[2] Internation Diabetes Federation, "About Diabetes," International Diabetes Federation, [Online]. Available: http://www.idf.org/about-diabetes. [Accessed 15 October 2014].

[3] BBC Science, "What is diabetes?," BBC, 19 April 2013. [Online]. Available: http://www.bbc.co.uk/science/0/21704103. [Accessed 111 2015].

[4] E. Gebel, "The Cost of Test Strips," Diabetes Forecast, July 2012. [Online]. Available: http://www.diabetesforecast.org/2012/jul/the-cost-of-test-strips.html. [Accessed 18 January 2015].

[5] Mayo Clinic Staff, "Blood sugar testing: Why, when and how," Mayo Clinic, 20 December 2014. [Online]. Available: http://www.mayoclinic.org/diseasesconditions/diabetes/in-depth/blood-sugar/art-20046628. [Accessed 18 January 2015].

[6] B. W. Carlos Eduardo Ferrante do Amaral, "Current development in non-invasive glucose monitoring," Medical Engineering and Physics, pp. 541-549, 2008.

[7] K. A. Wilson, "Re: What is the absorption spectrum of glucose?," MadSci Network, 18 February 2009. [Online]. Available: http://www.madsci.org/posts/archives/200902/1235057498.Bc.r.html. [Accessed 19 January 2015]. 
[8] H. Yu, D. Li, R. Roberts, K. Xu and N. Tien, "An Interstitial Fluid Transdermal Extraction System for Continuous Glucose Monitoring," Journal of Microelectromechanical Systems, pp. 917-925, August 2012.

[9] Metronic, "MiniMed Paradigm Revel Insulin Pump," Medtronic: For People Living with Diabetes, [Online]. Available: http://www.medtronicdiabetes.net/treatmentand-products/minimed-revel-insulin-pump. [Accessed 19 January 2015].

[10] A. AW, "The FDA Announces New, Tighter Home and Healthcare Facility Standards for Blood Glucose Meter Accuracy," diaTribe, 19 February 2014. [Online]. Available: http://diatribe.org/issues/61/new-now-next/3. [Accessed 20 January 2015].

[11] G. R. D.-C. M. Leboulanger B, "Reverse iontophoresis for non-invasive transdermal monitoring," Physiological Measurements, pp. R35-50, 2004.

[12] A. M. G. P. Andrea Tura, "Non-invasive glucose monitoring: Assessment of technologies," Diabetes Research and Clinical Practice, no. 77, pp. 16-40, January 2007.

[13] L. S. T. D. Sibbald RG, "Skin and Diabetes," Endocrinol Medab Clin North Am, vol. 2, no. 25, pp. 463-472, 1996.

[14] C. GL, "Noninvasive and minimally-invasive optical monitoring technologies," Journal of Nutrition, vol. 131, no. 5, pp. 159S-604S, 2001.

[15] Y. S. Y. I. K. T. Y. T. Yokota M, "A compact polarimetric glucose sensor using a high-performance fibre-optic Faraday rotator," Measurement Science and Technology, no. 15, pp. 143-147, 2004. 
[16] L. D. R. S. March W, "Fluorescent measurement in the non-invasive contact lens glucose sensor," Diabetes Technology and Therapeutics, no. 8, pp. 312-317, 2006.

[17] L. J. G. C. Badugu R, "A glucose-sensing contact lens: from bench top to patient," Current Opinion in Biotechnology, vol. 1, no. 16, pp. 100-107, 2005.

[18] R. G. David I Ellis, "Metabolic fingerprinting in disease diagnosis: biomedical applications of infrared and Raman spectroscopy," Analyst, vol. 131, no. 8, pp. 875$885,2006$.

[19] Y. O. K. H. M. K. K. Ok Kyung Cho, "Noninvasive measurement of glucose by metabolic heat conformation method," Clinical Chemistry, vol. 50, no. 10, pp. 1894$1898,2004$.

[20] K. S. J. L. J. B. Carl Malchoff, "A novel noninvasive blood glucose monitor," Diabetes Care, vol. 25, no. 12, pp. 2268-2275, 2002.

[21] V. N. J. D. M. P. N. B. S. Seungjun Lee, "Glucose measurements with sensors and ultrasound," Ultrasound in Medicine and Biology, vol. 31, no. 7, pp. 971-977, 2005.

[22] E. H. Y. F. Z. A. L. H. A Caduff, "First human experiments with a novel noninvasive, non-optical continuous glucose monitoring system," Biosensors and Bioelectronics, vol. 19, no. 3, pp. 209-217, 2003.

[23] R. A. G. R. N. M. J. F. Gourzi M, "Study of a new electromagnetic sensor for glycaemia measurement: in vitro results on blood pig," Journal of Medical Engineering and Technology, vol. 6, no. 27, pp. 276-281, 2003.

[24] The Engineering Toolbox, "Relative Permittivity - Dielectric Constant," The Engineering ToolBox, [Online]. Available: 
http://www.engineeringtoolbox.com/relative-permittivity-d_1660.html. [Accessed 14 January 2015].

[25] B. Freer, "FEASIBILITY OF A NON-INVASIVE WIRELESS BLOOD GLUCOSE MONITOR," Rochester Institute of Technology, Rochester, NY, 2011.

[26] S. O. T. H. M. Ada S. Y. Poon, "Optimal Frequency for Wireless Power Transmission Into Dispersive Tissue," IEEE Transactions on Antennas and Propagation, vol. 58, no. 5, pp. 1739-1750, 2010.

[27] A. Y. E. N. S. K. Y. t. B. P. R. B. A. P. John Ho, "Wireless power transfer to deeptissue microimplants," PNAS, vol. 111, no. 22, pp. 7974-7979, 2014.

[28] S. O. T. M. A.S.Y. Poon, "Optimal operating frequency in wireless power transmission for implantable devices," in IEEE International Conference of Engineering in Medicine and Biology, Lyon, France, 2007.

[29] R. L. C. G. S. Gabriel, "The dielectric properties of biological tissues: III. Parametric models for the dielectric spectrum of tissues," Phys. Med. Biol., vol. 41, no. 11, pp. 2271-2293, 1996.

[30] J. S. H. A. S. P. Sanghoek Kim, "Midfield wireless powering of subwavelength autonomous devices," Physical Review Letters, pp. 203905-1-5, 17 May 2013.

[31] K. Classic, "Radiofrequency (RF) Radiation," Health Physics Society, 13 August 2014. [Online]. Available: http://hps.org/hpspublications/articles/rfradiation.html. [Accessed 21 January 2015].

[32] Institute of Electrical and Electronic Engineers, IEEE standard for safety levels with respecto to human exposure to radio frequency electromagnetic fields, $3 \mathrm{kHz}$ to 300 
GHz (IEEE standard C95.1), New York: IEEE, 2005.

[33] E. C. M. E. T. Tutku Karacolak, "Cole-Cole Model for Glucose-Dependent Dielectric Properties of Blood Plasma for Continuous Glucose Monitoring," in International Union of Radio Science, Chicago, IL, 2008.

[34] Rogers Corporation, "RT/duroid® $5870 / 5880$ High Frequency Laminates," Rogers Corporation, 2014. [Online]. Available: http://www.rogerscorp.com/documents/606/acm/RT-duroid-5870-5880-DataSheet.pdf. [Accessed 12 January 2015].

[35] Rogers Corporation, "RO4000® Series High Frequency Circuit Materials," 2014. [Online]. Available: http://www.rogerscorp.com/documents/726/acm/RO4000Laminates---Data-sheet.pdf. [Accessed 26 November 2014].

[36] Amawave, "M17/133-00007," Amawave, 2015. [Online]. Available: http://amawave.com/ut-085-tp-m17.aspx. [Accessed 27 January 2015].

[37] D. Zhang, "EE500 Final Report," [Unpublished], San Luis Obispo, 2014.

[38] D. Pozar, Microwave Engineering, 4th Edition, Hoboken, NJ: John Wiley and Sons, Inc., 2012.

[39] Advanced Design System, Power Measurements, Keysight Technologies.

[40] Agilent Technologies, "Agilent AN-154 S-Parameter Design, Application Note," Agilent Technologies, Santa Rosa, CA, 2000.

[41] R. a. C. D.Andreuccetti, "An Internet resource for the calculation of the dielectric properties of body tissues in the frequency range $10 \mathrm{~Hz}-100 \mathrm{GHz}$," IFAC-CNR, 1997. [Online]. Available: http://niremf.ifac.cnr.it/tissprop/. [Accessed 22 October 
2014].

[42] Linear Technologies, "50MHz to 3GHz RF Power Detector with 60dB dynamic Range," 2014. [Online]. Available:

http://cds.linear.com/docs/en/datasheet/5534fc.pdf. [Accessed 22 January 2015].

[43] M. Nawrath, "Lab 3: Radio Signal Strength Sensor," Academy of Media Arts Cologne, March 2010. [Online]. Available:

http://interface.khm.de/index.php/lab/interfaces-advanced/radio-signal-strengthsensor/. [Accessed 24 October 2014].

[44] Urbana High School, "Electric Fields," [Online]. Available: www.usd116.org/lbeuschlein/physics/PowerPoint/electricfields.ppt. [Accessed 15 February 2015].

[45] "Coupled Line Couplers," Microwaves101.com, [Online]. Available: http://www.microwaves101.com/encyclopedias/coupled-line-couplers. [Accessed 1 February 2015].

[46] P. Bevelacqua, "Loop Antennas," Antenna-Theory.com, 2009. [Online]. Available: http://www.antenna-theory.com/antennas/smallLoop.php. [Accessed 27 January 2015].

[47] R. F. Y. H. Tuba Yilmaz, "Broadband Tissue Mimicking Phantoms and a Patch Resonator for Evaluating Noninvasive Monitoring of Blood Glucose Levels," IEEE Transactions on Antennas and Propagation, pp. 3064-3075, 6 June 2014.

[48] C. J. D. L. John V. Basmajian, Muscles Alive: Their Functions Revealed by Electromyography, Williams \& Wilkins, 1985. 
[49] International Diabetes Federation, "IDF Diabetes Atlas," International Diabetes

Federation, 2014. [Online]. Available: http://www.idf.org/diabetesatlas/update-2014. [Accessed 11 January 2015]. 


\title{
APPENDICES
}

\section{Fabricating Blood Mimicking Phantoms}

\author{
Created by Calvin Choy and Danielle Nishida
}

\section{Background}

Yilmaz, et al. developed broadband $(0-18 \mathrm{GHz})$ tissue mimicking phantoms for the purpose of testing noninvasive blood glucose monitors. Specific "recipes" were developed to produce phantoms mimicking wet skin, fat, blood, and muscle. Four different types of blood were created that matched blood glucose levels of 72, 126, 162, and $216 \mathrm{mg} / \mathrm{dL}$. The formula for blood phantoms were adjusted to create new blood phantoms for testing this sensor.

\section{Procedure}

Note: This procedure specifies measurements for producing a single batch of the blood mimicking phantom. Different measurements of dextrose are specified for creating different blood glucose levels.

Table 16: List of materials necessary for creating blood mimicking phantom.

\begin{tabular}{|rl|}
\hline & Ingredients \\
\hline $34.1 \mathrm{~g}$ & Gelatin \\
\hline $230 \mathrm{~g}$ & De-ionized water \\
\hline $1.2 \mathrm{~g}$ & NaCl \\
\hline $40 \mathrm{~g}$ & Ivory Ultra Dishwashing Detergent \\
\hline $15 \mathrm{~g}$ & Vegetable Oil \\
\hline (refer to Table 2) & Dextrose \\
\hline
\end{tabular}

1. Boil $230 \mathrm{~g}$ of de-ionized water and mix with $34.1 \mathrm{~g}$ of gelatin. Mix until consistency is homogenous (may need to remove some clumps after mixing for a minute).

2. Let rest for 3 minutes to allow mixture to cool.

3. Add $1.2 \mathrm{~g}$ of $\mathrm{NaCl}$ while stirring slowly.

4. Add the $40 \mathrm{~g}$ of room temperature dishwashing detergent while stirring slowly.

5. Let rest for 2 minutes to allow mixture to cool.

6. Add desired amount of dextrose according to Table 2.

Table 17: Measurements of dextrose necessary for achieving a variety of blood glucose levels.

\begin{tabular}{|c|c|}
\hline Blood Glucose Level [mg/dL] & Dextrose [g] \\
\hline 70 & 0.1397 \\
\hline
\end{tabular}




\begin{tabular}{|c|c|}
\hline 95 & 0.1896 \\
\hline 120 & 0.2395 \\
\hline 145 & 0.2894 \\
\hline 170 & 0.3393 \\
\hline 2505 & 5.0 \\
\hline
\end{tabular}

7. Add $15 \mathrm{~g}$ of room temperature oil, and mix until homogenous. (Mixture will become white at this point.) Let sit at room temperature for a few hours to solidify. 


\section{Results of Blood Mimicking Phantoms}

Upon testing the various blood mimicking phantoms, there was not a direct correlation between the glucose concentration and RSSI. The initial measurement of the pure blood phantom without glucose deviated in a predicted manner from the blood phantoms with glucose; however proper distinction between the blood phantoms with glucose could not be determined.

Table 18: Various blood phantoms were tested to fluctuate the received signal strength based on changing electrical properties.

\begin{tabular}{|c|c|c|c|}
\hline Number & Sample & $\begin{array}{c}\text { Glucose } \\
\text { Concentration } \\
\text { [mg/dL] }\end{array}$ & $\begin{array}{c}\text { RSSI } \\
{[\mathbf{d B m}]}\end{array}$ \\
\hline 1 & Air & 0 & -30.3 \\
\hline 2 & Blood 1 & 70 & -37.1 \\
\hline 3 & Blood 2 & 95 & -34.6 \\
\hline 4 & Blood 3 & 120 & -35.9 \\
\hline 5 & Blood 4 & 145 & -35.1 \\
\hline 6 & Blood 5 & 170 & -35.9 \\
\hline
\end{tabular}

Table 19: The expected behavior of RSSI based on different blood glucose levels was not demonstrated based on the blood phantom measurements.

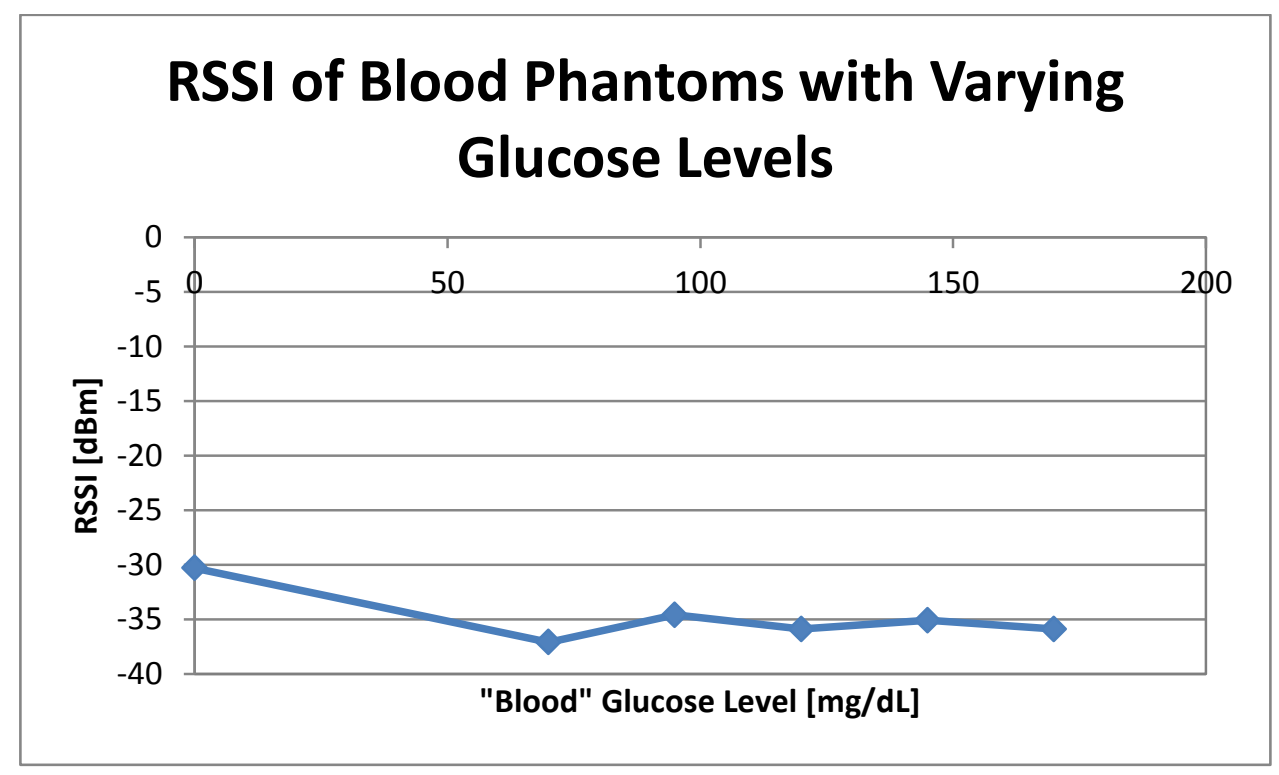

